\title{
2008
}

East Tennessee Technology Park Annual Illness and Injury Surveillance Report

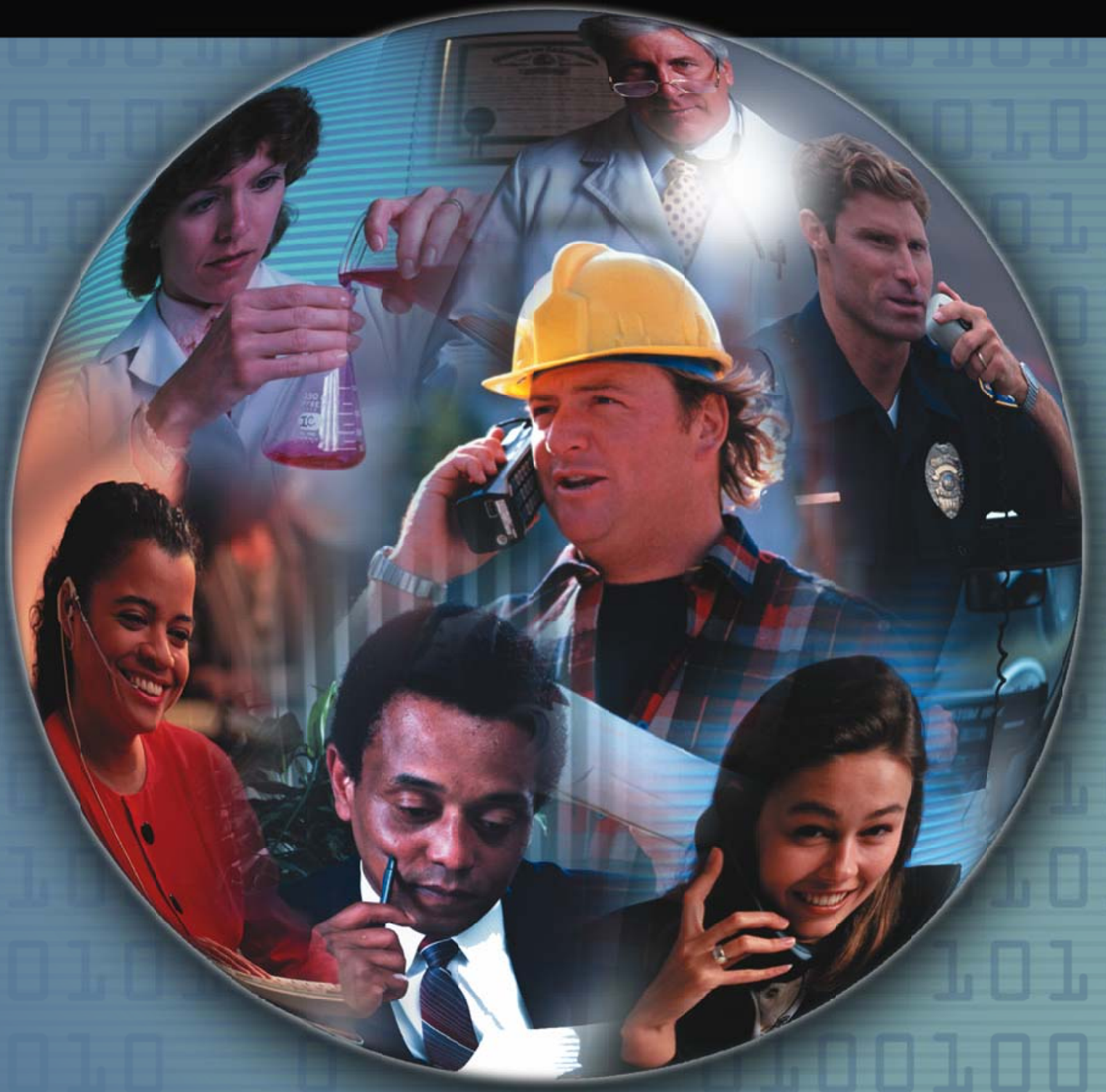




\section{East Tennessee Technology Park 2008 Illness and Injury Surveillance Report}

Questions or comments about this report or the Illness and Injury Surveillance Program (IISP) may be directed to:

E-mail:

Dr. Cliff Strader at cliff.strader@hq.doe.gov or Dr. Bonnie Richter at bonnie.richter@hq.doe.gov

or direct letters to:

Mail Stop HS-13 / 270CC

U.S. Department of Energy

1000 Independence Avenue, S.W.

Washington, DC 20585-0270

Additional information about the Department of Energy's Office of Illness and Injury Prevention Programs, the IISP, and annual reports for DOE sites participating in this program can be found at:

http://www.hss.energy.gov/healthsafety/WSHP/epi/surv/

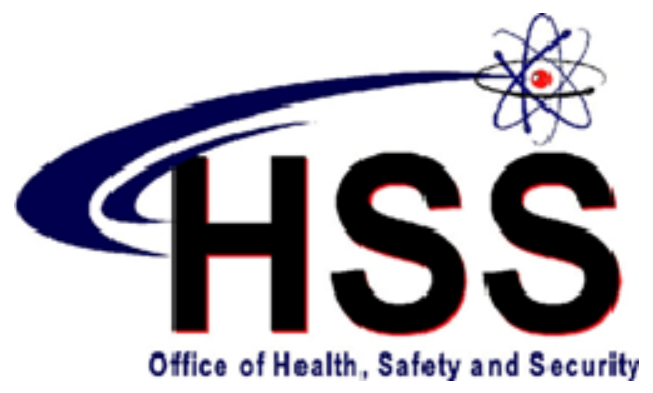

ACKNOWLEDGEMENT

LifeART images copyright 2000 Lippincott Williams \& Wilkins. All rights reserved.

This document was produced under contract number DE-AC05-06OR23100 between the

U.S. Department of Energy and Oak Ridge Associated Universities. 


\section{East Tennessee Technology Park \\ 2008 Illness and Injury Surveillance Report}

\section{At A Glance}

A total of 1,539 East Tennessee Technology Park (ETTP) employees were included in illness and injury surveillance in 2008, a decrease of 21 percent from 2007. The 2008 work force is 59 percent larger than the 2003 work force, despite the decrease in workers from 2007 to 2008. During this time, there has been an increase in male Service and Crafts workers and Line Operators; women Service workers also increased.

The average age of workers in 2008 was 47 years for women and 46 years for men. Since 2003, the average age for men has decreased and for women has been relatively stable. The decrease in average age of men from 2003 to 2008 is the result of hiring workers less than 30 years of age. In 2003, the percentage of male workers less than 30 years of age was 3 percent; in 2008, 16 percent of men were in this age group. We did not see this shift in age among women, but we did see an increase in women aged 50 and older, which suggests that the female work force has been fairly stable and aging.

Women reported 32 absences, resulting in an absence rate of 8.5 absences per 100 workers (32/377). The 1,162 men reported 66 absences, yielding an absence rate of 5.7 per 100 workers $(66 / 1,162)$. The overall absence rate declined one-third from 2005 to 2008. The types of conditions reported during this time have not changed among men; however, among women there has been a shift away from injuries and respiratory conditions and an increase in psychological and genitourinary disorders. The higher rate of absences among women compared with men has been generally seen among all DOE sites participating in the Illness and Injury Surveillance Program (IISP).

Security and Fire workers had the highest absence rate among men. Among women, the Technical Support workers had the highest absence rate in 2008.

Among the 90 diagnoses reported by men, musculoskeletal conditions and injuries accounted for 44 percent. Among women, 46 percent of the 48 diagnoses were for musculoskeletal conditions, genitourinary disorders, and psychological conditions. 
No definite occupational sentinel health events (SHEOs) were identified in 2008. The 3 possible SHEOs were for carpal tunnel syndrome reported by 3 workers in the Service and Administrative Support job categories, all aged 40 years or older.

Women reported 5 OSHA-recordable events and men reported 27 OSHA-recordable events in 2008. Thirty-one of the 32 diagnoses were for injuries, mainly resulting from sprains and strains and open wounds. Seven OSHA-recordable events reported by men were the result of accidents. Three of the accidents were due to a cutting/piercing instrument/object with 1 each the result of being struck by an object or exposure to hot, corrosive, or caustic material/steam. No accidents were reported by women.

After declining to a minimum of 9.5 events per 1,000 workers in 2004, the rate of OSHA events more than doubled to 20.8 events per 1,000 workers in 2008. The overall trend among sites participating in IISP has been a decline in OSHA event rates, but this trend has not been seen at ETTP. The increased rate from 2004 through 2008 could reflect inexperience among newer workers in the work force and the type of work being performed. 


\section{The East Tennessee Technology Park Work Force - 2008}

The Work Force by Gender and Age ............. 1

The Work Force by Gender and Job

Category 1

Number and Length of Absences

Absence Rate by Gender and Age 2

Number of Days Absent by

Gender and Age

Absence Rate by Job Category

and Gender 3

Average Duration of Absence by

Job Category and Gender. .. 3

Diagnostic Categories

Number of Diagnoses and Lost Calendar Days by Diagnostic Category

(Categorized by ICD-9-CM) and Gender . .4

Common Diagnoses Among Female

Workers in 2008 .. 5

Common Diagnoses Among Male

Workers in 2008 6

Number of Most Frequently Reported

Diagnoses by Job Category and Gender 7

\section{Rates of Disease Occurrence}

Rates for All Illnesses and Injuries Combined by Job Category, Gender, and Age

Rates for Selected Diagnostic Categories by Job Category, Gender, and Age .8

\section{Time Trends}

Age-Adjusted Rates for All Diagnoses Combined Among Women and Men from 2003 to 2008
Age-Adjusted Rates for Selected Diagnostic Categories Among Women and Men from 2003 to 2008

Age-Adjusted Rates for All Diagnoses

Combined Among Women and Men by Job

Category from 2003 to $2008 \ldots \ldots \ldots \ldots \ldots \ldots \ldots \ldots . \ldots 12$

\section{Sentinel Health Events for Occupations (SHEOs)}

Characteristics of SHEOs by Gender 13

SHEO Diagnoses by Gender 13

Occupational Safety and Health Administration (OSHA)-Recordable Events

OSHA-Recordable Events by Gender and Age. 14

OSHA-Recordable Events by Job

Category and Gender 14

\section{Diagnostic and Accident Categories for OSHA-Recordable Events}

OSHA-Recordable Diagnoses by

Diagnostic Category and Gender

OSHA-Recordable Accidents by Type

and Gender

Rates of OSHA-Recordable Events

OSHA-Recordable Rates by Age and Job

Categories Among Women, All Diagnoses

Combined

OSHA-Recordable Rates by Age and Job

Categories Among Men, All Diagnoses

Combined

Time Trends for OSHA-Recordable Events

Age-Adjusted Rates for All OSHA-Recordable Diagnoses Combined Among Women and Men by Job Category from 2003 to 2008 .

\section{Appendices}


The East Tennessee Technology Park Work Force - 2008

Figure 1. The Work Force by Gender and Age

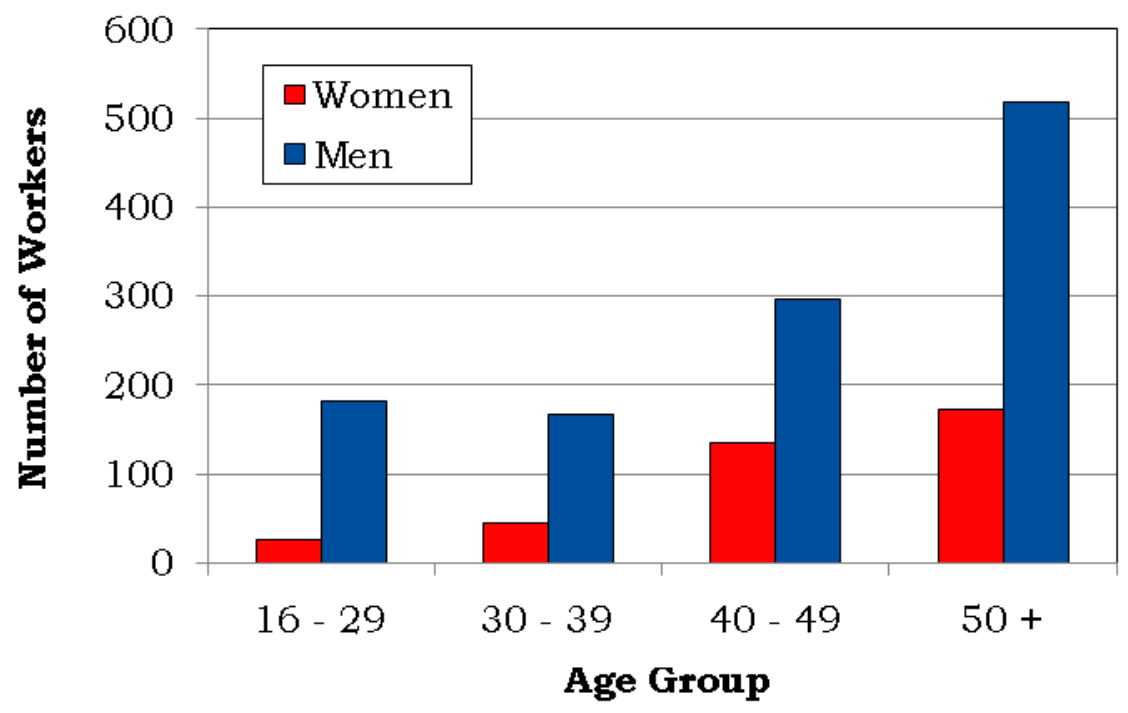

Figure 2. The Work Force by Gender and Job Category

\begin{tabular}{|l|c|c|}
\hline \multicolumn{1}{|c|}{ Job Category } & W omen & M en \\
\hline Professional & 108 & 389 \\
& $29 \%$ & $33 \%$ \\
\hline \multirow{2}{*}{ Administrative Support } & 114 & 39 \\
& $30 \%$ & $3 \%$ \\
\hline \multirow{2}{*}{ Technical Support } & 7 & $\mathbf{8}$ \\
& $2 \%$ & $1 \%$ \\
\hline Service & 130 & 367 \\
& $34 \%$ & $32 \%$ \\
\hline \multirow{2}{*}{ Security and Fire } & 2 & 21 \\
& $1 \%$ & $2 \%$ \\
\hline \multirow{2}{*}{ Crafts } & $\mathbf{8}$ & 217 \\
& $2 \%$ & $19 \%$ \\
\hline \multirow{2}{*}{ Line Operators } & $\mathbf{8}$ & 121 \\
& $2 \%$ & $10 \%$ \\
\hline \multirow{2}{*}{ Total } & 377 & $\mathbf{1 , 1 6 2}$ \\
& $100 \%$ & $100 \%$ \\
\hline
\end{tabular}




\section{Number and Length of Absences}

Figure 3. Absence Rate by Gender and Age

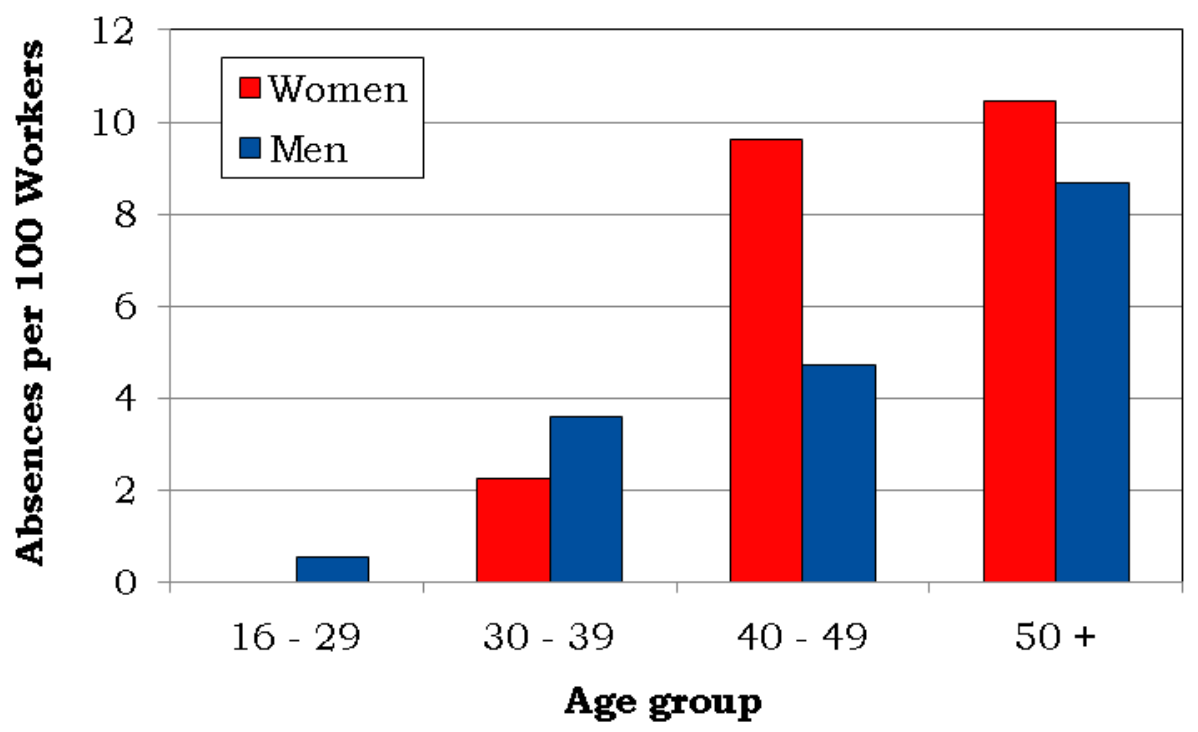

Figure 4. Number of Days Absent by Gender and Age

\begin{tabular}{|c|c|c|c|c|}
\hline \multirow{3}{*}{ Gender } & \multirow{2}{*}{ Age } & \multirow{2}{*}{$\begin{array}{c}\text { Number of } \\
\text { Absences }\end{array}$} & \multicolumn{2}{|c|}{ Number of Days Absent } \\
\cline { 3 - 5 } & & & Total & Average \\
\hline \multirow{4}{*}{ Women } & $16-29$ & 0 & 0 & 0 \\
\cline { 2 - 5 } & $30-39$ & 1 & 136 & 136 \\
\cline { 2 - 5 } & $40-49$ & 13 & 545 & 42 \\
\cline { 2 - 5 } & $50+$ & 18 & 1,199 & 67 \\
\cline { 2 - 5 } & Total & 32 & 1,880 & 59 \\
\hline \multirow{4}{*}{ Men } & $16-29$ & 1 & 31 & 31 \\
\cline { 2 - 5 } & $30-39$ & 6 & 246 & 41 \\
\cline { 2 - 5 } & $40-49$ & 14 & 664 & 47 \\
\cline { 2 - 5 } & $50+$ & 45 & 2,150 & 48 \\
\cline { 2 - 5 } & Total & 66 & 3,091 & 47 \\
\hline
\end{tabular}


Figure 5. Absence Rate by Job Category and Gender

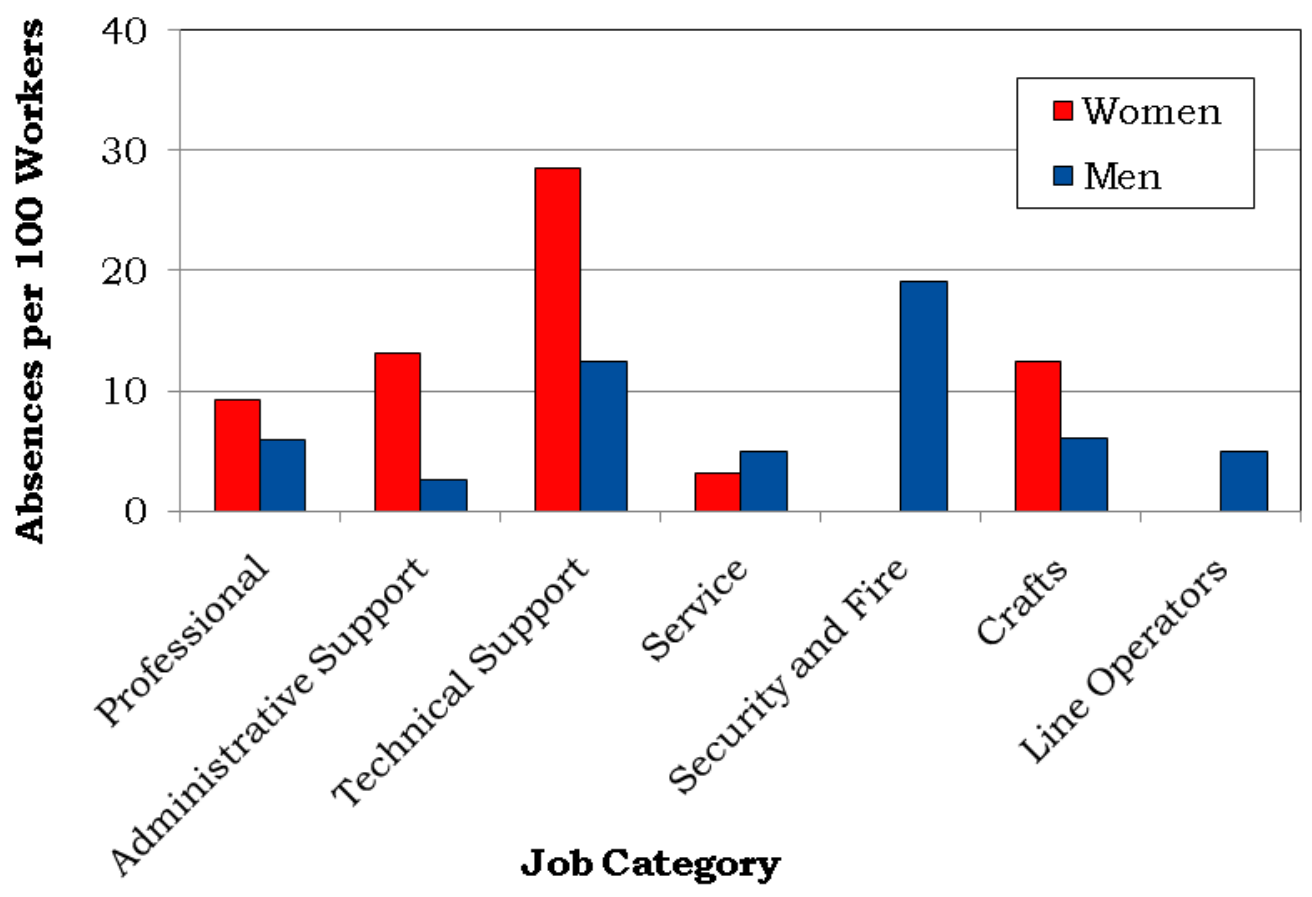

Figure 6. Average Duration of Absence by Job Category and Gender

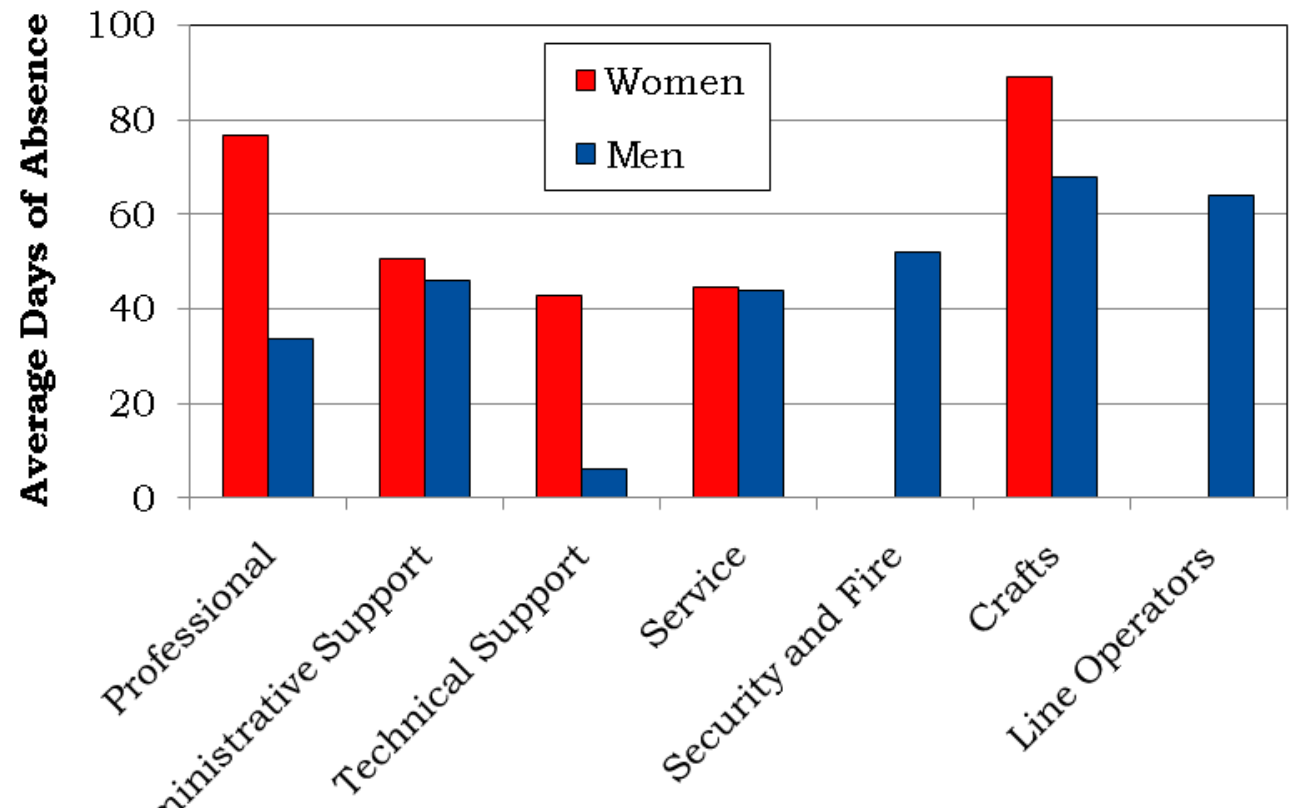

Job Category 


\section{Diagnostic Categories}

Figure 7. Number of Diagnoses and Lost Calendar Days by Diagnostic Category (Categorized by ICD-9-CM) and Gender

\begin{tabular}{|l|c|c|c|c|}
\hline \multirow{2}{*}{ Diagnostic Category } & \multicolumn{2}{c|}{ Women } & \multicolumn{2}{c|}{ M en } \\
\cline { 2 - 5 } & $\begin{array}{c}\text { Number of } \\
\text { Diagnoses }\end{array}$ & $\begin{array}{c}\text { Number } \\
\text { of Lost } \\
\text { Calendar } \\
\text { Days }\end{array}$ & $\begin{array}{c}\text { Number of } \\
\text { Diagnoses }\end{array}$ & $\begin{array}{c}\text { Number } \\
\text { of Lost } \\
\text { Calendar } \\
\text { Days }\end{array}$ \\
\hline Benign Growths & $\mathbf{1}$ & $\mathbf{1 3}$ & $\mathbf{0}$ & $\mathbf{0}$ \\
\hline Blood & $\mathbf{0}$ & $\mathbf{0}$ & $\mathbf{0}$ & $\mathbf{0}$ \\
\hline Cancer & $\mathbf{0}$ & $\mathbf{0}$ & $\mathbf{4}$ & $\mathbf{3 2 8}$ \\
\hline Digestive & $\mathbf{4}$ & $\mathbf{9 8}$ & $\mathbf{1 0}$ & $\mathbf{7 2}$ \\
\hline Endocrine/Metabolic & $\mathbf{3}$ & $\mathbf{3 9 1}$ & $\mathbf{1}$ & $\mathbf{1 7}$ \\
\hline Existing Birth Condition & $\mathbf{0}$ & $\mathbf{0}$ & $\mathbf{2}$ & $\mathbf{7 1}$ \\
\hline Genitourinary & $\mathbf{6}$ & $\mathbf{1 7 2}$ & $\mathbf{1}$ & $\mathbf{1 4}$ \\
\hline Heart/Circulatory & $\mathbf{4}$ & $\mathbf{1 6}$ & $\mathbf{7}$ & $\mathbf{6 9 5}$ \\
\hline Infections/Parasites & $\mathbf{0}$ & $\mathbf{0}$ & $\mathbf{1}$ & $\mathbf{2 3}$ \\
\hline Injury & $\mathbf{4}$ & $\mathbf{1 6 3}$ & $\mathbf{1 6}$ & $\mathbf{5 7 5}$ \\
\hline Miscarriage & $\mathbf{0}$ & $\mathbf{0}$ & $\mathbf{N A}$ & NA \\
\hline Musculoskeletal & $\mathbf{1 1}$ & $\mathbf{1 , 0 7 2}$ & $\mathbf{2 4}$ & $\mathbf{1 , 1 6 8}$ \\
\hline Nervous System & $\mathbf{3}$ & $\mathbf{8 5}$ & $\mathbf{5}$ & $\mathbf{2 6 8}$ \\
\hline Psychological & $\mathbf{5}$ & $\mathbf{3 1 5}$ & $\mathbf{0}$ & $\mathbf{0}$ \\
\hline Respiratory & $\mathbf{3}$ & $\mathbf{3 0}$ & $\mathbf{1 3}$ & $\mathbf{6 4}$ \\
\hline Skin & $\mathbf{0}$ & $\mathbf{0}$ & $\mathbf{0}$ & $\mathbf{0}$ \\
\hline Unspecified Symptoms & $\mathbf{6 0}$ & $\mathbf{6}$ & $\mathbf{4 0 9}$ \\
\hline
\end{tabular}

Note: Lost calendar days for each absence are counted more than once when multiple diagnoses occur in different diagnostic categories for the same absence. 


\section{Figure 8. Common Diagnoses Among Female Workers in 2008}

Intestinal Disorders

Hernias

Gallbladder

Disorders Esophagus/Stomach Appendicitis

All Other

Kidney Disorders

Disorders of the Reproductive Organs

Bladder Disorders

All Other

Sprains and Strains

Open Wounds

Fractures

Dislocations

Bruises

All Other

Rheumatism

Joints

Back

All Other

Upper Respiratory

Pneumonia/Flu

Bronchitis/Asthma

All Other

Headache

Fatigue

Dizziness

Digestive Symptoms

Chest Pain

All Other
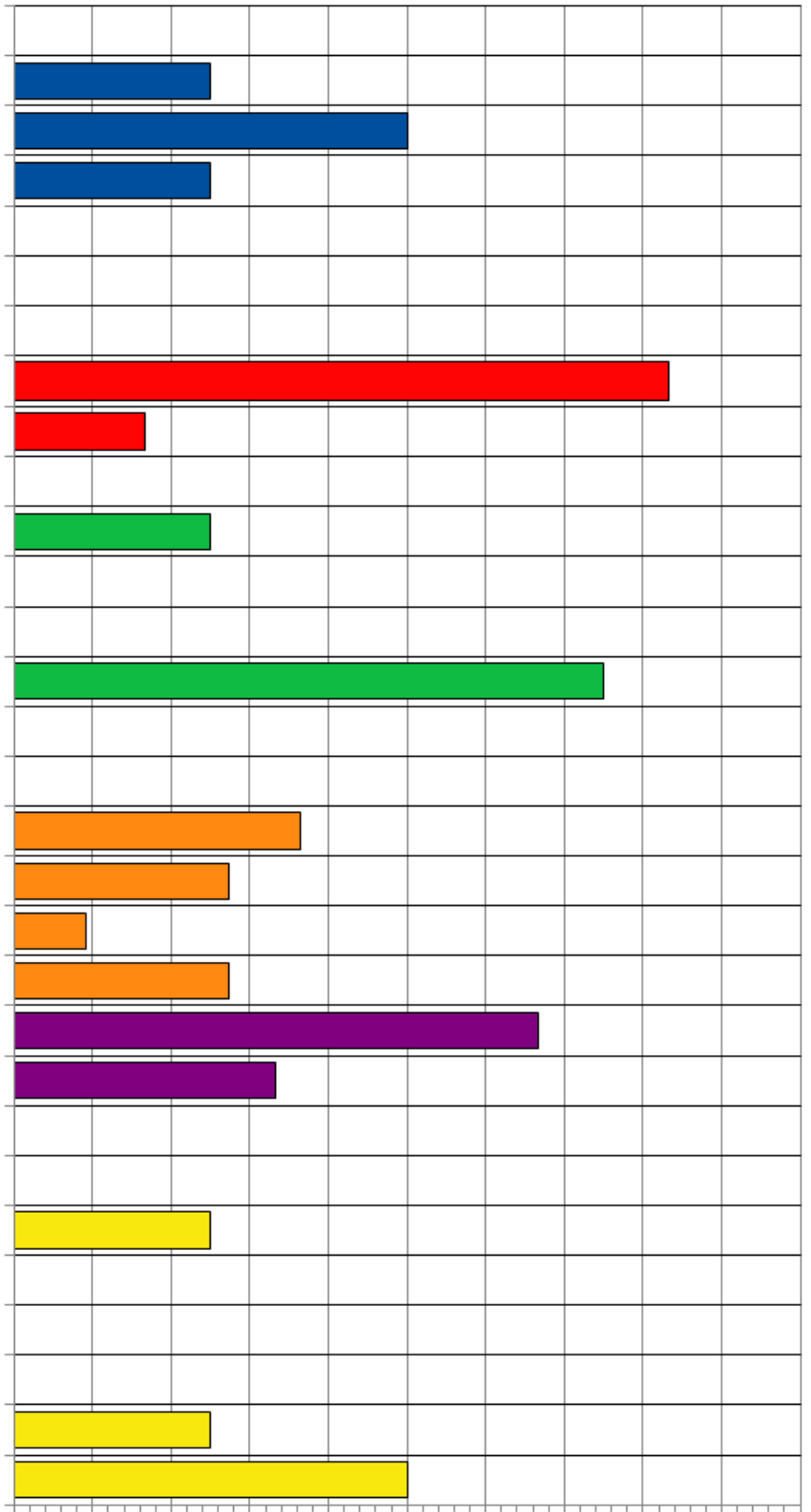

$\begin{array}{lllllllllll}0 & 10 & 20 & 30 & 40 & 50 & 60 & 70 & 80 & 90 & 100\end{array}$

Percent Distribution of Diagnoses Within Diagnostic Category

Dige stive, 4 Diagnoses

Genitourinary, 6 Diagnoses

Injury, 4 Diagnoses
Musculoskeletal, 11 Diagnoses

Respiratory, 3 Diagnoses

Unspecified Symptoms, 4 Diagnoses 
Figure 9. Common Diagnoses Among Male Workers in 2008

Intestinal Disorders

Hernias

Gallbladder

Disorders Esophagus/Stomach

Appendicitis

All Other

Kidney Disorders

Disorders of the Reproductive Organs

Bladder Disorders

All Other

Sprains and Strains

Open Wounds

Fractures

Dislocations

Bruises

All Other

Rheumatism

Joints

Back

All Other

Upper Respiratory

Pneumonia/Flu

Bronchitis / Asthma

All Other

Headache

Fatigue

Dizziness

Digestive Symptoms

Chest Pain

All Other
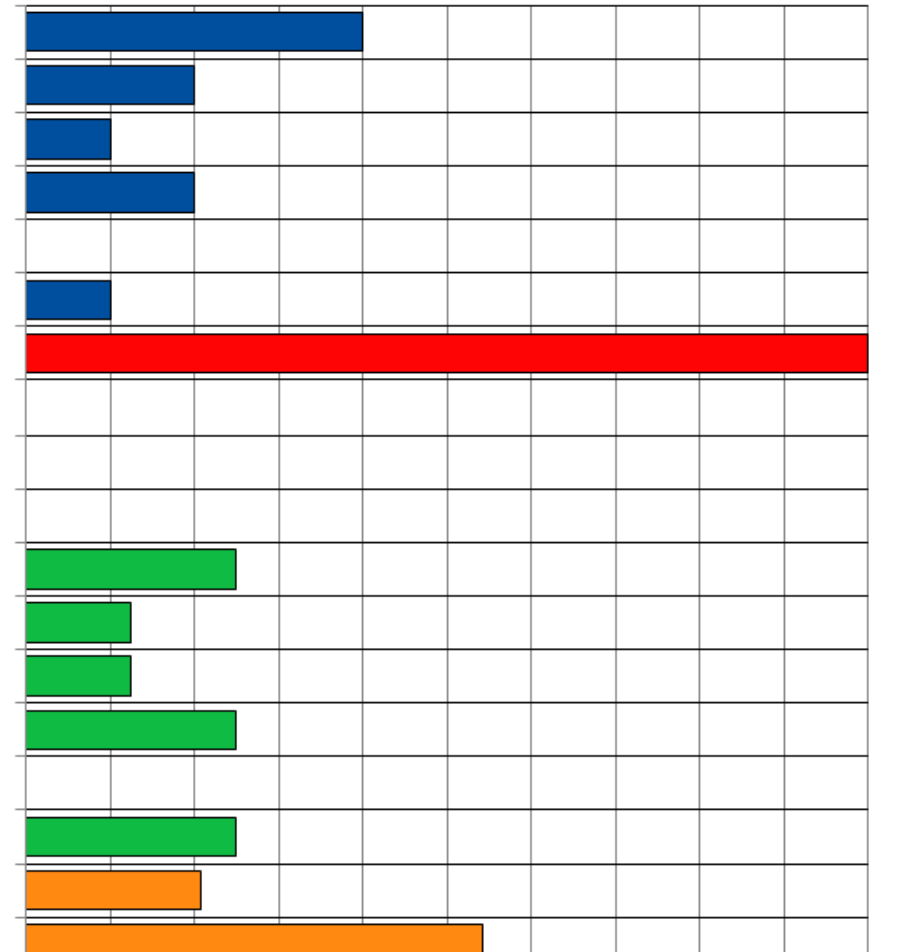

$=$$$
=
$$

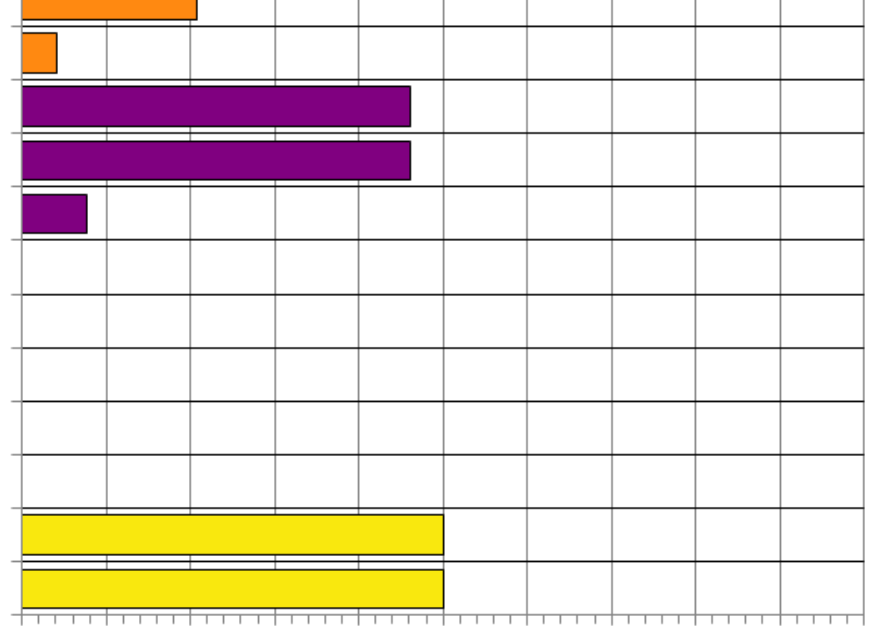

$\begin{array}{lllllllllll}0 & 10 & 20 & 30 & 40 & 50 & 60 & 70 & 80 & 90 & 100\end{array}$

Percent Distribution of Diagnoses Within Diagnostic Category

Digestive, 10 Diagnoses

Genitourinary, 1 Diagnosis

Injury, 16 Diagnoses
Musculoskeletal, 24 Diagnoses

- Respiratory, 13 Diagnoses

Unspecified Symptoms, 6 Diagnoses 


\section{Figure 10. Number of Most Frequently Reported Diagnoses by Job Category and Gender}

\begin{tabular}{|c|c|c|c|c|}
\hline Job Category & Men & & Women & \\
\hline \multirow{5}{*}{ Professional } & M usculoskeletal & 12 & M usculoskeletal & 5 \\
\hline & Injury & 7 & Genitourinary & 2 \\
\hline & Respiratory & 5 & Unspecified Symptoms & 2 \\
\hline & Digestive & 5 & Digestive & 2 \\
\hline & & & Endocrine/M etabolic & 2 \\
\hline \multirow{5}{*}{ Administrative Support } & Existing Birth Condition & 1 & M usculoskeletal & 3 \\
\hline & & & Genitourinary & 3 \\
\hline & & & Psychological & 3 \\
\hline & & & Heart/Circulatory & 3 \\
\hline & & & Nervous System & 3 \\
\hline \multirow{2}{*}{ Technical Support } & M usculoskeletal & 2 & M usculoskeletal & 2 \\
\hline & & & Injury & 2 \\
\hline \multirow{5}{*}{ Service } & M usculoskeletal & 5 & Genitourinary & 1 \\
\hline & Injury & 4 & Unspecified Symptoms & 1 \\
\hline & Nervous System & 3 & Psychological & 1 \\
\hline & & & Respiratory & 1 \\
\hline & & & Heart/Circulatory & 1 \\
\hline \multirow{3}{*}{ Security and Fire } & Respiratory & 4 & & 0 \\
\hline & Digestive & 2 & & \\
\hline & Cancer & 1 & & \\
\hline \multirow{4}{*}{ Crafts } & M usculoskeletal & 4 & M usculoskeletal & 1 \\
\hline & Injury & 2 & & \\
\hline & Heart/Circulatory & 2 & & \\
\hline & Unspecified Symptoms & 2 & & \\
\hline \multirow{3}{*}{ Line Operators } & Injury & 3 & & 0 \\
\hline & Respiratory & 2 & & \\
\hline & Digestive & 2 & & \\
\hline
\end{tabular}




\section{Rates of Disease Occurrence}

Figure 11. Rates for All Illnesses and Injuries Combined by Job Category, Gender, and Age

\begin{tabular}{|c|c|c|c|c|}
\hline \multirow{2}{*}{$\begin{array}{c}\text { All Illnesses \& } \\
\text { Injuries Combined }\end{array}$} & \multicolumn{4}{|c|}{ Rate per 1,000} \\
\hline & Job Category & Age & Men & Women \\
\hline & \multirow{2}{*}{ Professional } & $<50$ & 72 & 140 \\
\hline & & $50+$ & 100 & 157 \\
\hline & \multirow{2}{*}{ Administrative Support } & $<50$ & 50 & 23 \\
\hline & & $50+$ & 0 & 300 \\
\hline & \multirow{2}{*}{ Technical Support } & $<50$ & 0 & 800 \\
\hline & & $50+$ & 667 & 0 \\
\hline & \multirow{2}{*}{ Service } & $<50$ & 26 & 56 \\
\hline & & $50+$ & 121 & 0 \\
\hline & \multirow{2}{*}{ Security and Fire } & $<50$ & 636 & 0 \\
\hline & & $50+$ & 0 & 0 \\
\hline & \multirow{2}{*}{ Crafts } & $<50$ & 22 & 167 \\
\hline & & $50+$ & 145 & 0 \\
\hline & \multirow{2}{*}{ Line Operators } & $<50$ & 29 & 0 \\
\hline & & $50+$ & 170 & 0 \\
\hline
\end{tabular}

Figure 12. Rates for Selected Diagnostic Categories by Job Category, Gender, and Age

\begin{tabular}{|c|c|c|c|c|}
\hline \multirow{2}{*}{ Cancer } & \multicolumn{4}{|c|}{ Rate per 1,000} \\
\hline & Job Category & Age & Men & Women \\
\hline & Professional & $<50$ & 0 & 0 \\
\hline & & $50+$ & 4 & 0 \\
\hline & Adminictrative Sunmet & $<50$ & 0 & 0 \\
\hline & 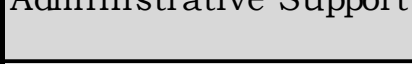 & $50+$ & 0 & 0 \\
\hline & Technical Support & $<50$ & 0 & 0 \\
\hline & & $50+$ & 0 & 0 \\
\hline & Service & $<50$ & 0 & 0 \\
\hline & 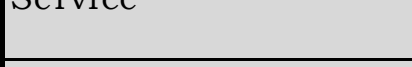 & $50+$ & 10 & 0 \\
\hline & Security and Fire & $<50$ & 91 & 0 \\
\hline & 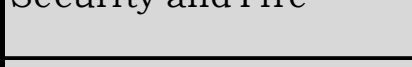 & $50+$ & 0 & 0 \\
\hline & Crafts & $<50$ & 0 & 0 \\
\hline & & $50+$ & 12 & 0 \\
\hline & Line Oper & $<50$ & 0 & 0 \\
\hline & & $50+$ & 0 & 0 \\
\hline
\end{tabular}


Figure 12. Rates for Selected Diagnostic Categories by Job Category, Gender, and Age (Continued)

\begin{tabular}{|l|l|c|c|c|}
\hline \multirow{2}{*}{ Heart/Circulatory } & \multicolumn{4}{|c|}{ Rate per 1,000 } \\
\cline { 2 - 5 } & Job Category & Age & M en & W omen \\
\cline { 2 - 5 } & \multirow{2}{*}{ Profe ssional } & $<50$ & $\mathbf{0}$ & $\mathbf{0}$ \\
\cline { 2 - 5 } & $50+$ & $\mathbf{8}$ & $\mathbf{0}$ \\
\cline { 2 - 5 } & \multirow{2}{*}{ Administrative Support } & $<50$ & $\mathbf{0}$ & $\mathbf{0}$ \\
\cline { 2 - 5 } & & $50+$ & $\mathbf{0}$ & $\mathbf{4 3}$ \\
\cline { 2 - 5 } & \multirow{2}{*}{ Technical Support } & $<50$ & $\mathbf{0}$ & $\mathbf{0}$ \\
\cline { 2 - 5 } & & $50+$ & $\mathbf{0}$ & $\mathbf{0}$ \\
\cline { 2 - 5 } & \multirow{2}{*}{ Service } & $<50$ & $\mathbf{0}$ & $\mathbf{1 1}$ \\
\cline { 2 - 5 } & $50+$ & $\mathbf{2 0}$ & $\mathbf{0}$ \\
\cline { 2 - 5 } & \multirow{2}{*}{ Security and Fire } & $<50$ & $\mathbf{0}$ & $\mathbf{0}$ \\
\cline { 2 - 5 } & $50+$ & $\mathbf{0}$ & $\mathbf{0}$ \\
\cline { 2 - 5 } & \multirow{2}{*}{ Crafts } & $<50$ & $\mathbf{0}$ & $\mathbf{0}$ \\
\cline { 2 - 5 } & $50+$ & $\mathbf{2 4}$ & $\mathbf{0}$ \\
\cline { 2 - 5 } & \multirow{2}{*}{ Line Operators } & $<50$ & $\mathbf{0}$ & $\mathbf{0}$ \\
\cline { 2 - 5 } & $50+$ & $\mathbf{1 9}$ & $\mathbf{0}$ \\
\hline
\end{tabular}

\begin{tabular}{|c|c|c|c|c|}
\hline \multirow{2}{*}{ Respiratory } & \multicolumn{4}{|c|}{ Rate per 1,000} \\
\hline & Job Category & Age & Men & Women \\
\hline & \multirow{2}{*}{ Professional } & $<50$ & 0 & 18 \\
\hline & & $50+$ & 20 & 0 \\
\hline & \multirow{2}{*}{ Administrative Support } & $<50$ & 0 & 0 \\
\hline & & $50+$ & 0 & 14 \\
\hline & \multirow{2}{*}{ Technical Support } & $<50$ & 0 & 0 \\
\hline & & $50+$ & 0 & 0 \\
\hline & \multirow{2}{*}{ Service } & $<50$ & 0 & 11 \\
\hline & & $50+$ & 10 & 0 \\
\hline & \multirow{2}{*}{ Security and Fire } & $<50$ & 364 & 0 \\
\hline & & $50+$ & 0 & 0 \\
\hline & \multirow{2}{*}{ Crafts } & $<50$ & 0 & 0 \\
\hline & & $50+$ & 12 & 0 \\
\hline & \multirow{2}{*}{ Line Operators } & $<50$ & 29 & 0 \\
\hline & & $50+$ & 0 & 0 \\
\hline
\end{tabular}


Figure 12. Rates for Selected Diagnostic Categories by Job Category, Gender, and Age (Continued)

\begin{tabular}{|c|c|c|c|c|}
\hline \multirow{2}{*}{ Injury } & \multicolumn{4}{|c|}{ Rate per 1,000} \\
\hline & Job Category & Age & Men & Women \\
\hline & \multirow{2}{*}{ Professional } & $<50$ & 22 & 18 \\
\hline & & $50+$ & 16 & 0 \\
\hline & \multirow{2}{*}{ Administrative Support } & $<50$ & 0 & 0 \\
\hline & & $50+$ & 0 & 14 \\
\hline & \multirow{2}{*}{ Technical Support } & $<50$ & 0 & 400 \\
\hline & & $50+$ & 0 & 0 \\
\hline & \multirow{2}{*}{ Service } & $<50$ & 7 & 0 \\
\hline & & $50+$ & 20 & 0 \\
\hline & \multirow{2}{*}{ Security and Fire } & $<50$ & 0 & 0 \\
\hline & & $50+$ & 0 & 0 \\
\hline & \multirow{2}{*}{ Crafts } & $<50$ & 7 & 0 \\
\hline & & $50+$ & 12 & 0 \\
\hline & \multirow{2}{*}{ Line Operators } & $<50$ & 0 & 0 \\
\hline & & $50+$ & 57 & 0 \\
\hline
\end{tabular}

\section{Time Trends}

Figure 13. Age-Adjusted Rates for All Diagnoses Combined Among Women and Men from 2003 to 2008*

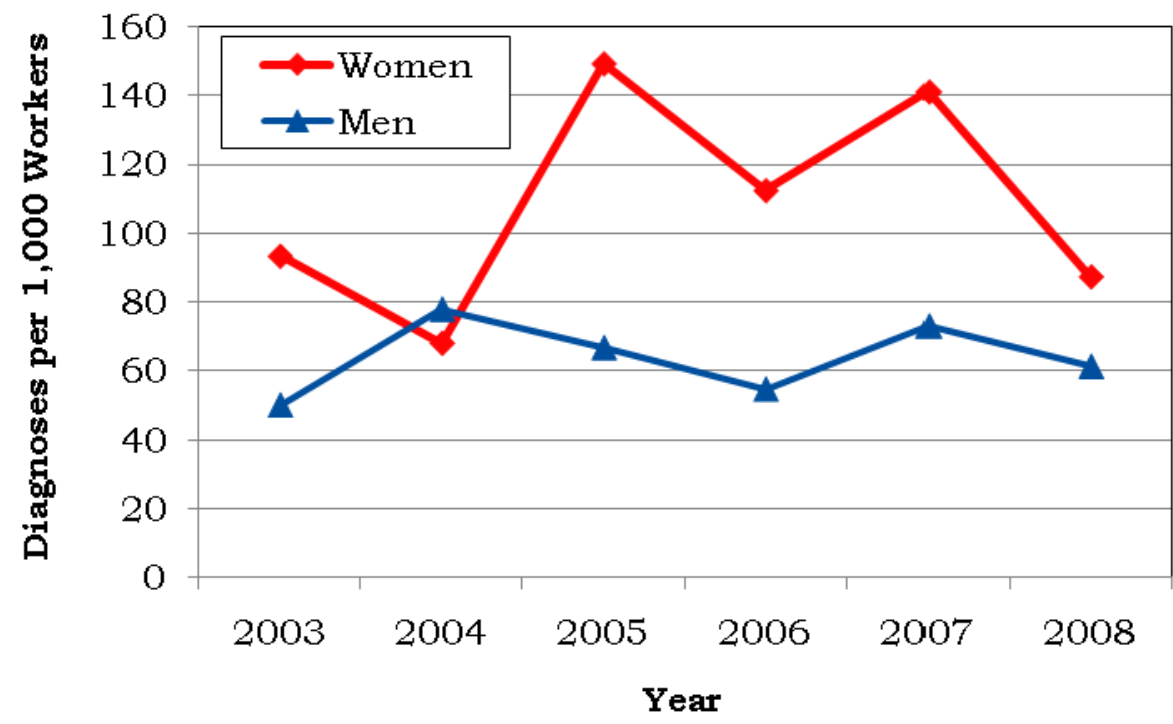

*Standardized to age distribution of 2000 U.S. population. 
Figure 14. Age-Adjusted Rates for Selected Diagnostic Categories Among Women and Men from 2003 to 2008*

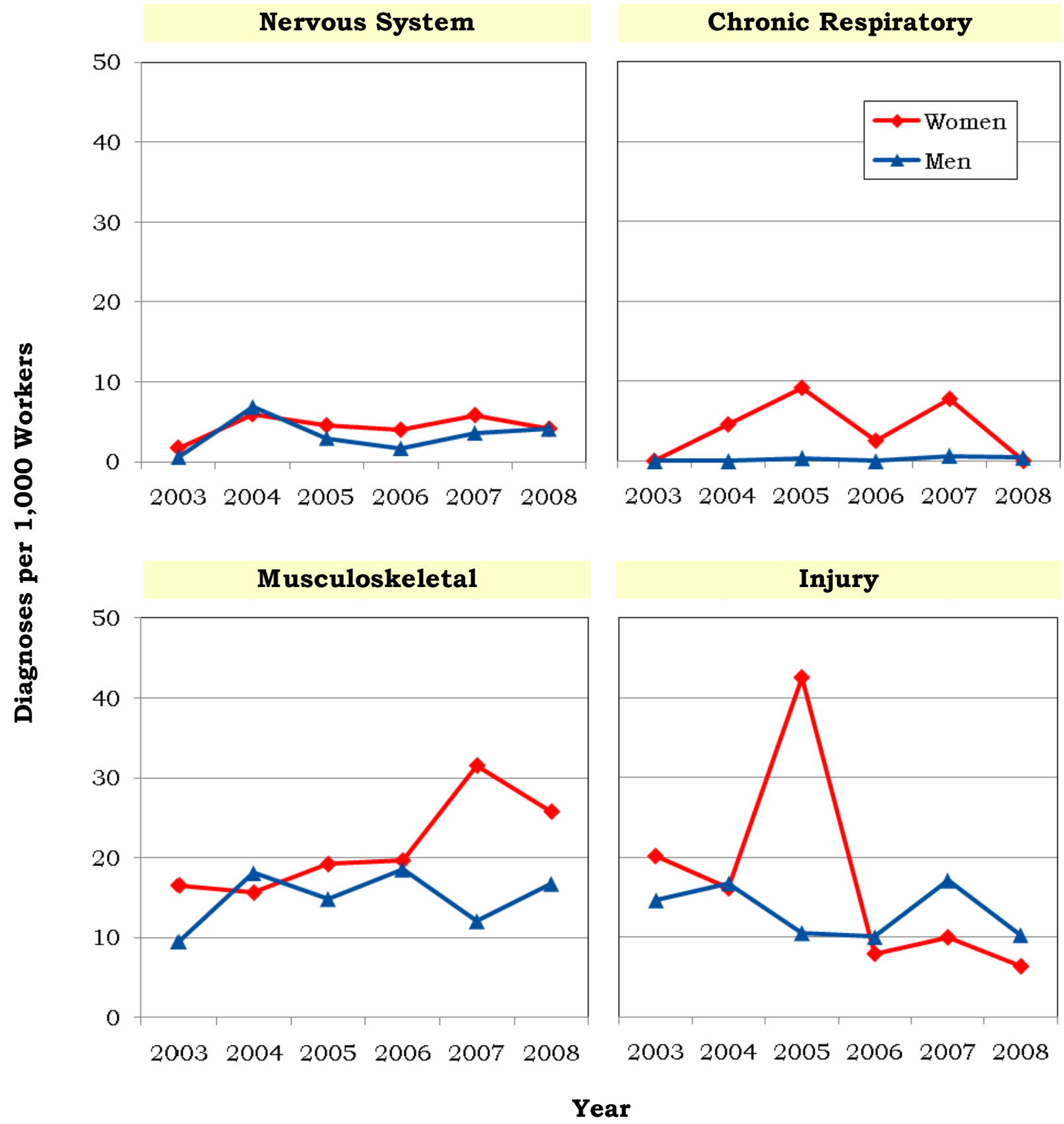

*Standardized to age distribution of 2000 U.S. population. 
Figure 15. Age-Adjusted Rates for All Diagnoses Combined Among Women and Men by Job Category from 2003 to 2008*
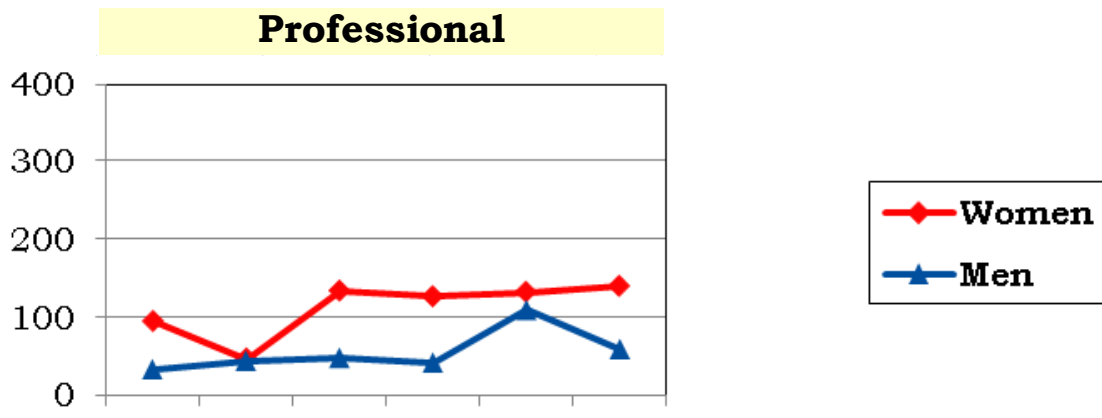

200320042005200620072008

Administrative Support

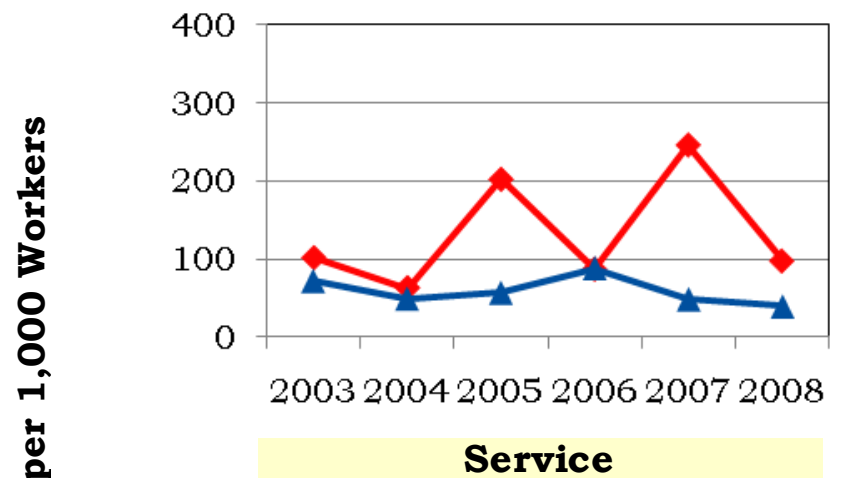

\section{Technical Support}

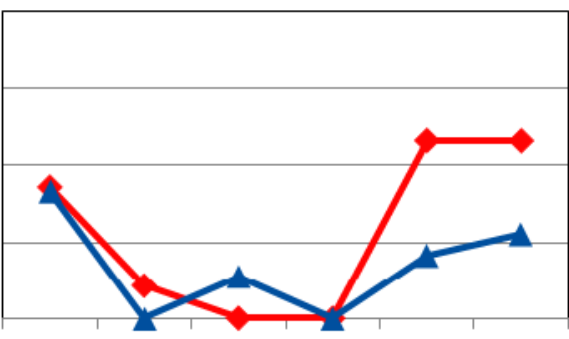

200320042005200620072008

Security and Fire

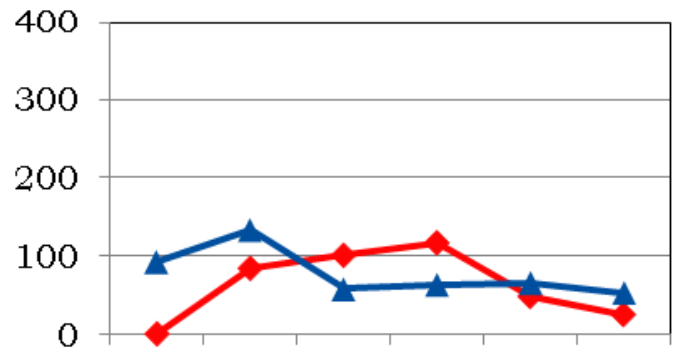

200320042005200620072008

\section{Crafts}
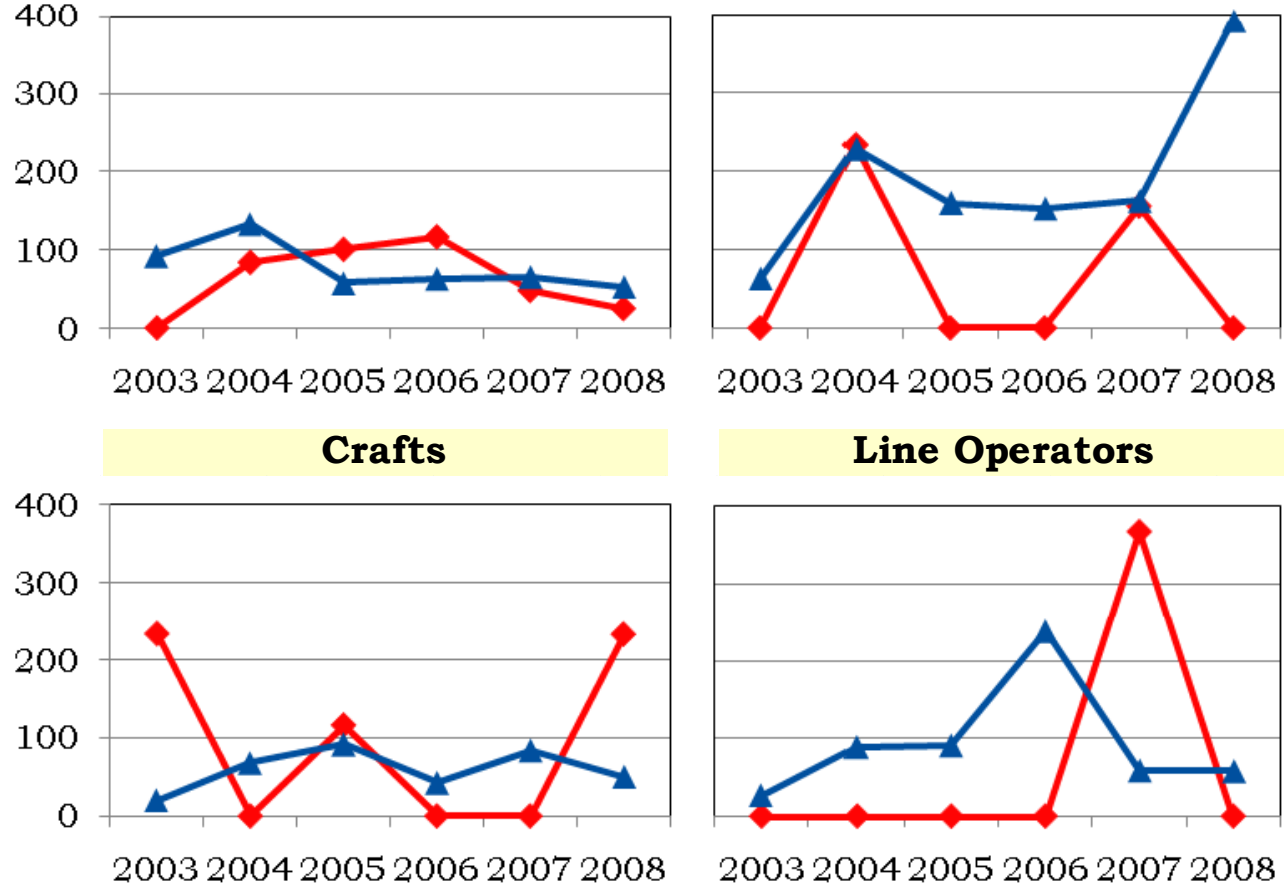

200320042005200620072008

Line Operators

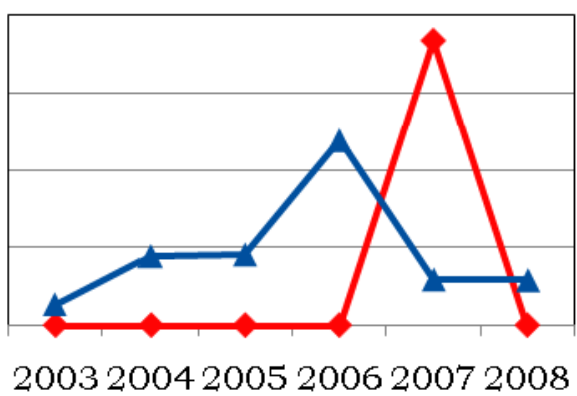

Year

*Standardized to age distribution of 2000 U.S. population. 


\section{Sentinel Health Events for Occupations (SHEOs)}

An occupational sentinel health event (SHEO) is a disease, disability, or death that is likely to be occupationally related. Although sentinel health events may indicate an occupational exposure, many may result from nonoccupational exposures. Sentinel health events are therefore assessed in two categories:

Definite Sentinel Health Events: Diseases that are unlikely to occur in the absence of an occupational exposure (e.g., asbestosis).

Possible Sentinel Health Events: Diseases that may be occupational but can also occur in the absence of an occupational exposure (e.g., lung cancer or carpal tunnel syndrome).

Figure 16. Characteristics of SHEOs by Gender

\begin{tabular}{|l|c|c|c|c|}
\hline \multirow{2}{*}{} & \multicolumn{2}{|c|}{$\begin{array}{c}\text { Total Number of } \\
\text { SHEO Diagnoses }\end{array}$} & \multicolumn{2}{c|}{$\begin{array}{c}\text { Total Number of } \\
\text { Days Absent }\end{array}$} \\
\cline { 2 - 5 } & Men & Women & Men & W omen \\
\hline Definite & 0 & 0 & 0 & 0 \\
\hline Possible & 2 & 1 & 50 & 19 \\
\hline Total & 2 & 1 & 50 & 19 \\
\hline
\end{tabular}

Figure 17. SHEO Diagnoses by Gender

\begin{tabular}{|l|c|c|}
\hline \multirow{2}{*}{ Diagnoses } & \multicolumn{2}{|c|}{ Gender } \\
\cline { 2 - 3 } & Women & M en \\
\hline Carpal Tunnel Syndrome & 1 & 2 \\
\hline
\end{tabular}


Occupational Safety and Health Administration (OSHA)-Recordable Events

Figure 18. OSHA-Recordable Events by Gender and Age

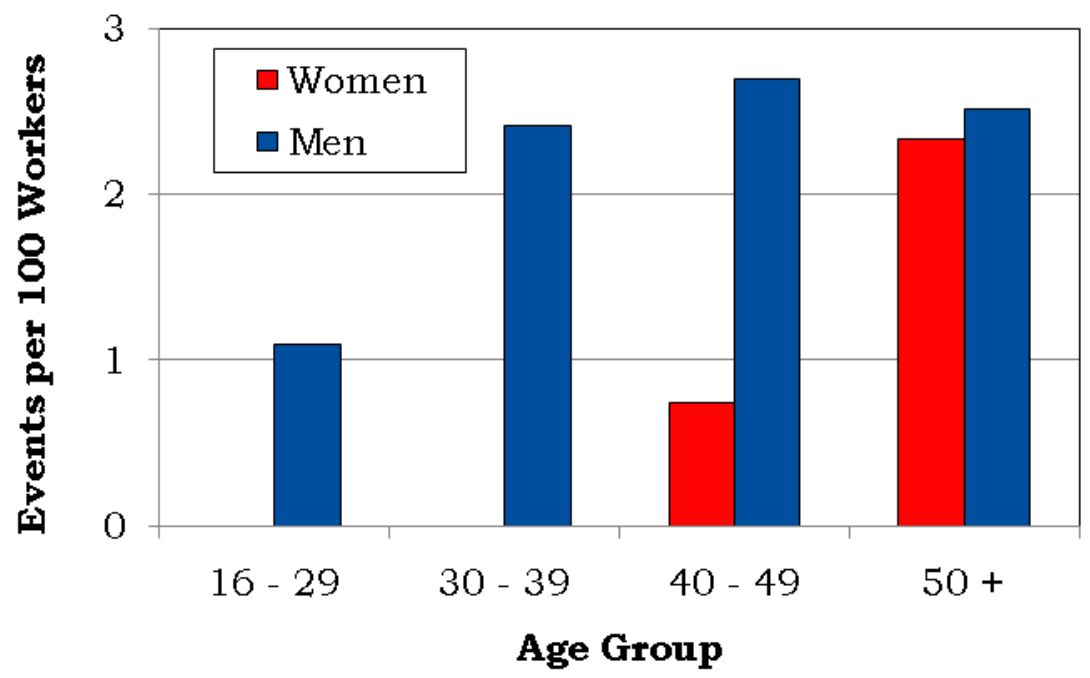

Figure 19. OSHA-Recordable Events by Job Category and Gender

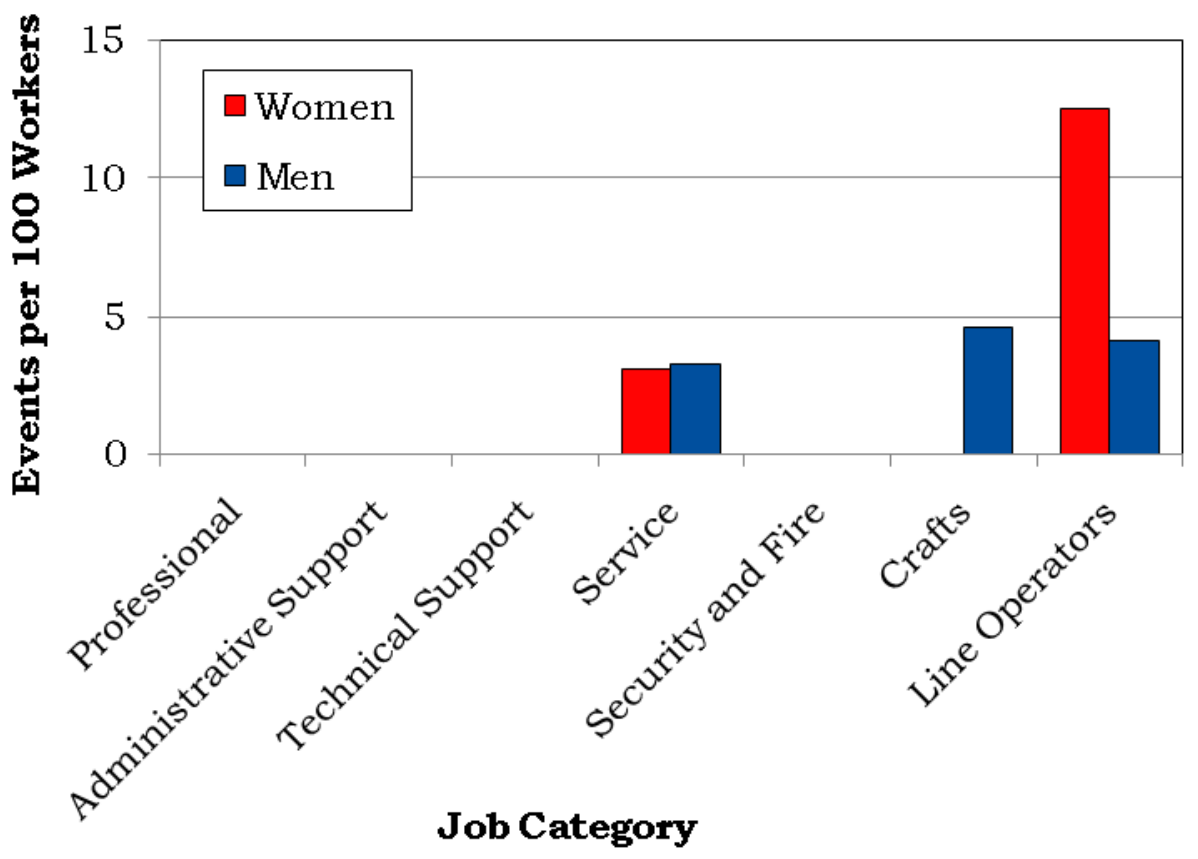




\section{Diagnostic and Accident Categories for OSHA-Recordable Events}

Figure 20. OSHA-Recordable Diagnoses by Diagnostic Category and Gender

\begin{tabular}{|l|c|c|}
\hline \multirow{2}{*}{ Diagnostic Category } & \multicolumn{2}{|c|}{ Gender } \\
\cline { 2 - 3 } & Women & M en \\
\hline Nervous System & $\mathbf{0}$ & $\mathbf{1}$ \\
\hline Injury & $\mathbf{5}$ & $\mathbf{2 6}$ \\
\hline Fractures - Lower Limb & $\mathbf{0}$ & $\mathbf{1}$ \\
\hline Back Sprains \& Strains & $\mathbf{0}$ & $\mathbf{2}$ \\
\hline Other Sprains \& Strains & $\mathbf{3}$ & $\mathbf{9}$ \\
\hline Open Wounds - Head, Neck, Trunk & $\mathbf{0}$ & $\mathbf{3}$ \\
\hline Open Wounds - Upper Limb & $\mathbf{1}$ & $\mathbf{4}$ \\
\hline Open Wounds - Lower Limb & $\mathbf{0}$ & $\mathbf{2}$ \\
\hline Superficial Injuries & $\mathbf{0}$ & $\mathbf{3}$ \\
\hline Bruises & $\mathbf{1}$ & $\mathbf{1}$ \\
\hline Burns & $\mathbf{0}$ & $\mathbf{1}$ \\
\hline
\end{tabular}

Figure 21. OSHA-Recordable Accidents by Type and Gender

\begin{tabular}{|l|c|c|}
\hline \multirow{2}{*}{ Accident Category } & \multicolumn{2}{c|}{ Gender } \\
\cline { 2 - 3 } & Women & Men \\
\cline { 2 - 3 } & $\begin{array}{c}\text { Number of } \\
\text { Accidents }\end{array}$ & $\begin{array}{c}\text { Number of } \\
\text { Accidents }\end{array}$ \\
\hline Natural/Environmental Factors & $\mathbf{0}$ & $\mathbf{2}$ \\
\hline Other Accidents & $\mathbf{0}$ & $\mathbf{5}$ \\
\hline Struck by an Object & $\mathbf{0}$ & $\mathbf{1}$ \\
\hline Cutting/Piercing Instrument/Object & $\mathbf{0}$ & $\mathbf{3}$ \\
\hline Hot, Corrosive, or Caustic Material/Steam & $\mathbf{0}$ & $\mathbf{1}$ \\
\hline Total & $\mathbf{0}$ & $\mathbf{7}$ \\
\hline
\end{tabular}




\section{Rates of OSHA-Recordable Events}

Figure 22. OSHA-Recordable Rates by Age and Job Categories Among Women, All Diagnoses Combined

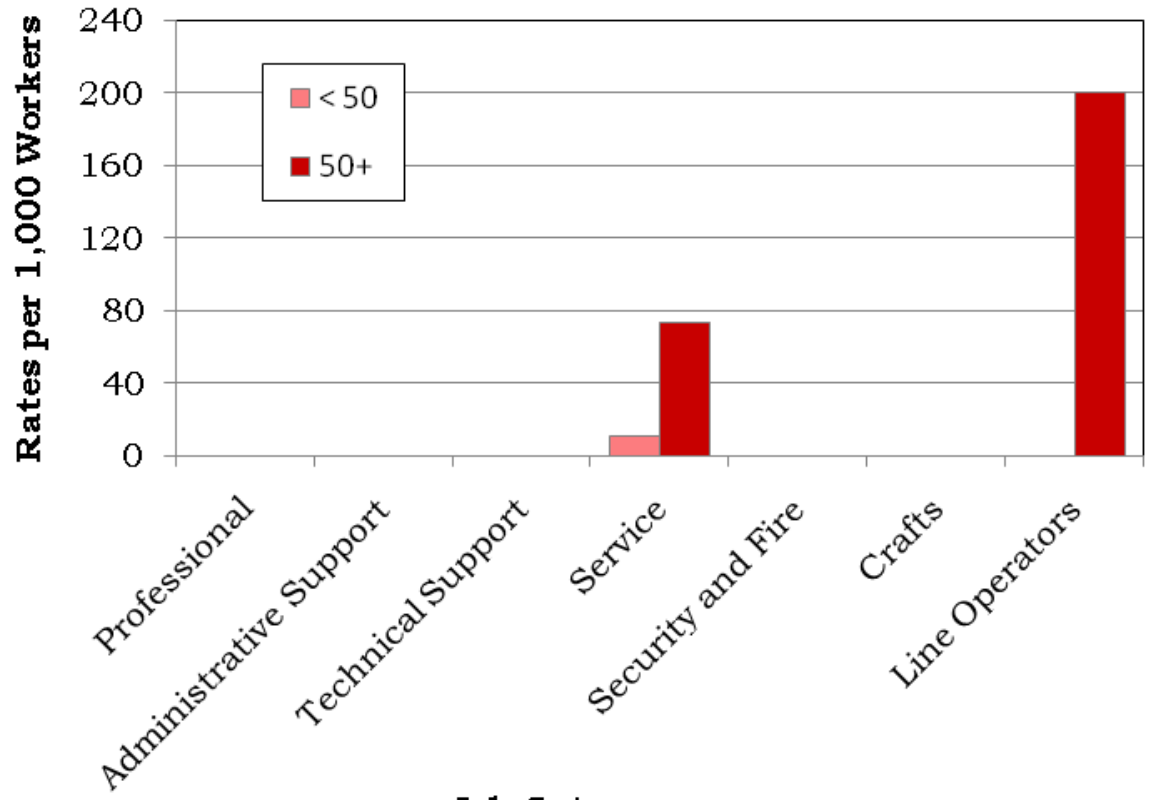

Job Category

Figure 23. OSHA-Recordable Rates by Age and Job Categories Among Men, All Diagnoses Combined

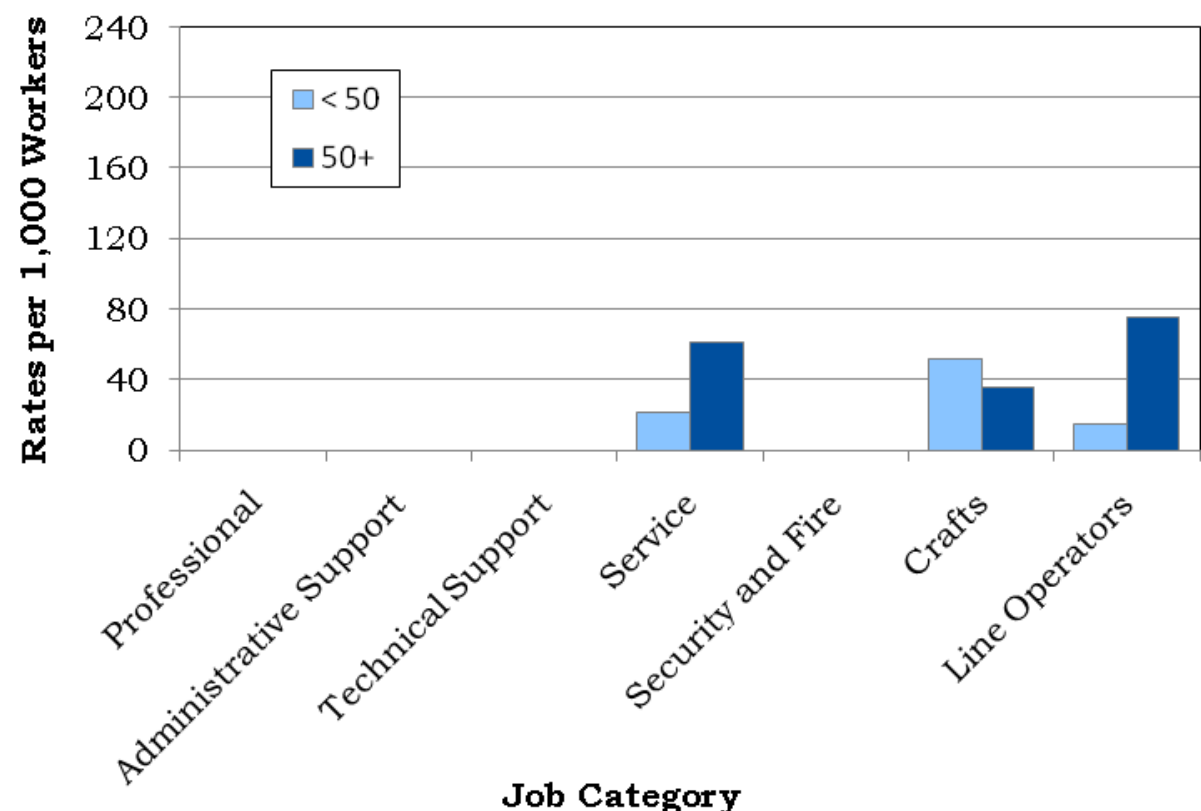




\section{Time Trends for OSHA-Recordable Events}

Figure 24. Age-Adjusted Rates for All OSHA-Recordable Diagnoses Combined Among Women and Men by Job Category from 2003 to 2008*

Professional

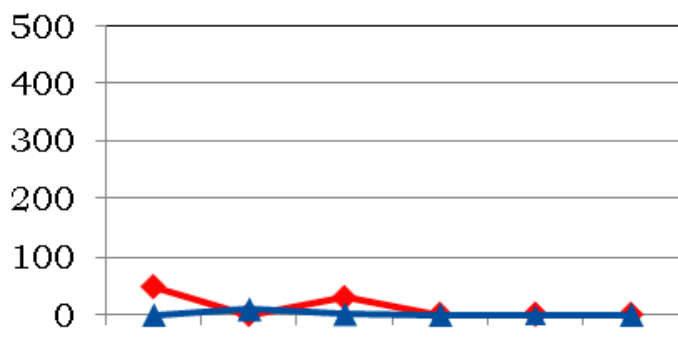

200320042005200620072008

Administrative Support

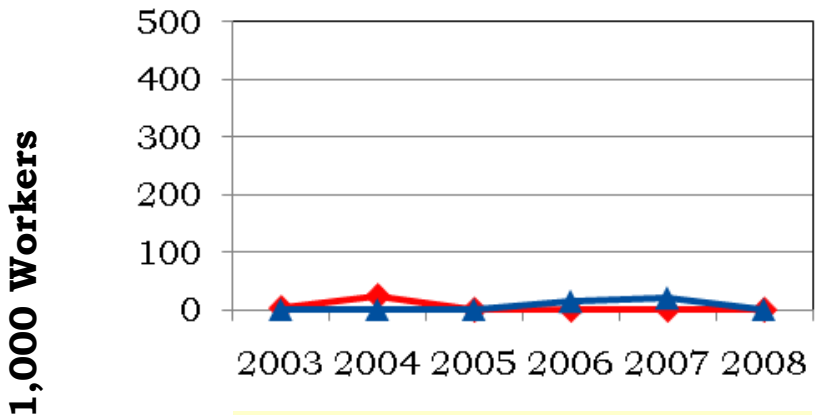

Service

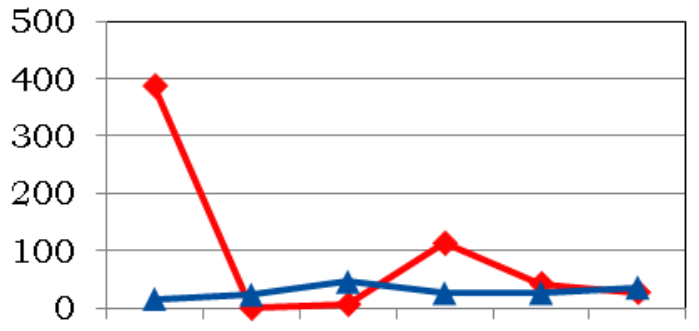

200320042005200620072008

Crafts

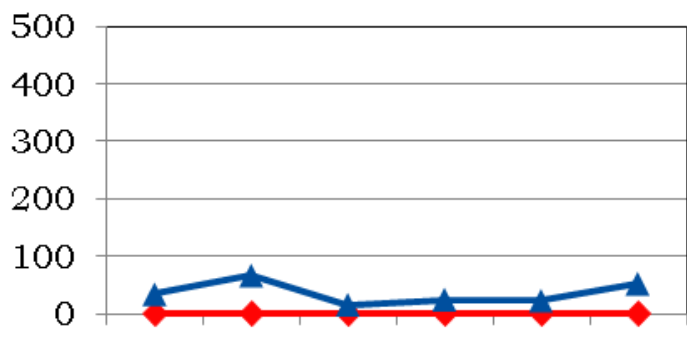

200320042005200620072008

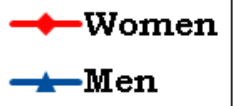

Technical Support

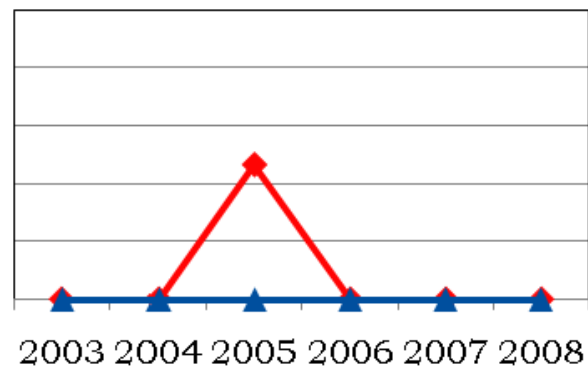

Security and Fire

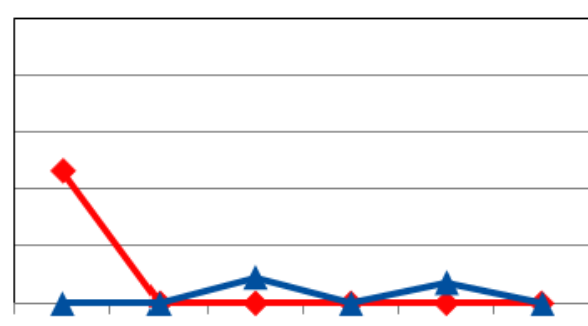

200320042005200620072008

Line Op erators

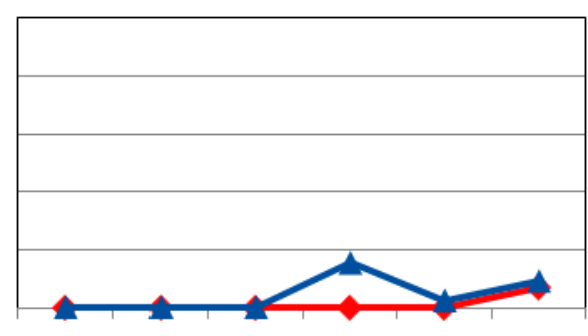

200320042005200620072008

Year

*Standardized to age distribution of 2000 U.S. population. 
Appendices 
East Tennessee Technology Park 2008

Absence Data

Appendix A. Work Force by Gender, Age, and Job Category

\begin{tabular}{|c|c|c|c|c|c|c|c|c|c|c|c|}
\hline \multirow{3}{*}{ Job Category } & \multicolumn{5}{|c|}{ Women } & \multicolumn{5}{|c|}{ Men } & \multirow[b]{3}{*}{ TOTAL } \\
\hline & \multicolumn{4}{|c|}{ Age Group } & \multirow[b]{2}{*}{ TOTAL } & \multicolumn{4}{|c|}{ Age Group } & \multirow[b]{2}{*}{ TOTAL } & \\
\hline & $16-29$ & $30-39$ & $40-49$ & $50+$ & & 16 - 29 & 30 - 39 & $40-49$ & $50+$ & & \\
\hline Professional & 3 & 5 & 49 & 51 & 108 & 13 & 20 & 105 & 251 & 389 & 497 \\
\hline Administrative Support & 2 & 11 & 31 & 70 & 114 & 7 & 6 & 7 & 19 & 39 & 153 \\
\hline Technical Support & 0 & 1 & 4 & 2 & 7 & 0 & 2 & 3 & 3 & 8 & 15 \\
\hline Service & 18 & 24 & 47 & 41 & 130 & 100 & 81 & 87 & 99 & 367 & 497 \\
\hline Security and Fire & 0 & 1 & 0 & 1 & 2 & 2 & 3 & 6 & 10 & 21 & 23 \\
\hline Crafts & 3 & 2 & 1 & 2 & 8 & 44 & 30 & 60 & 83 & 217 & 225 \\
\hline Line Operators & 0 & 0 & 3 & 5 & 8 & 15 & 24 & 29 & 53 & 121 & 129 \\
\hline TOTAL & 26 & 44 & 135 & 172 & 377 & 181 & 166 & 297 & 518 & 1,162 & 1,539 \\
\hline
\end{tabular}

Appendix B. Age Distribution of the Work Force by Gender

\begin{tabular}{|l|r|r|r|r|r|r|r|r|}
\hline \multirow{3}{*}{ Year } & \multicolumn{4}{|c|}{ Women } & \multicolumn{4}{c|}{ Men } \\
\cline { 2 - 9 } & \multicolumn{1}{|c|}{ Percent Distribution by Age Group } & \multicolumn{1}{c|}{ Percent Distribution by Age Group } \\
\cline { 2 - 9 } & $\mathbf{1 6}-\mathbf{2 9}$ & $\mathbf{3 0}-\mathbf{3 9}$ & $\mathbf{4 0 - 4 9}$ & $\mathbf{5 0}+$ & $\mathbf{1 6}-\mathbf{2 9}$ & $\mathbf{3 0}-\mathbf{3 9}$ & $\mathbf{4 0}-\mathbf{4 9}$ & $\mathbf{5 0}+$ \\
\hline $\mathbf{1 9 9 9}$ & 5.50 & 27.02 & 43.53 & 23.95 & 2.10 & 15.08 & 39.76 & 43.06 \\
\hline $\mathbf{2 0 0 0}$ & 3.29 & 23.04 & 45.57 & 28.10 & 1.16 & 13.53 & 38.27 & 47.04 \\
\hline $\mathbf{2 0 0 1}$ & 3.19 & 22.36 & 43.13 & 31.31 & 1.39 & 11.44 & 33.69 & 53.48 \\
\hline $\mathbf{2 0 0 2}$ & 4.09 & 20.75 & 42.14 & 33.02 & 3.97 & 11.15 & 30.69 & 54.20 \\
\hline $\mathbf{2 0 0 3}$ & 4.69 & 19.06 & 42.19 & 34.06 & 3.24 & 10.96 & 33.02 & 52.78 \\
\hline $\mathbf{2 0 0 4}$ & 4.88 & 19.54 & 40.36 & 35.22 & 11.00 & 13.87 & 27.34 & 47.79 \\
\hline $\mathbf{2 0 0 5}$ & 6.62 & 17.89 & 41.18 & 34.31 & 13.95 & 14.30 & 27.13 & 44.62 \\
\hline $\mathbf{2 0 0 6}$ & 6.87 & 17.52 & 35.25 & 40.35 & 14.70 & 15.82 & 25.67 & 43.81 \\
\hline $\mathbf{2 0 0 7}$ & 7.36 & 16.67 & 35.50 & 40.48 & 15.34 & 15.95 & 24.73 & 43.99 \\
\hline $\mathbf{2 0 0 8}$ & 6.90 & 11.67 & 35.81 & 45.62 & 15.58 & 14.29 & 25.56 & 44.58 \\
\hline
\end{tabular}


East Tennessee Technology Park 2008

Absence Data

Appendix C. Total Number of Workers Who Reported at Least One Absence by Gender, Age, and Job Category*

\begin{tabular}{|c|c|c|c|c|c|c|c|c|c|c|}
\hline \multirow{3}{*}{ Job Category } & \multicolumn{4}{|c|}{ Women } & \multicolumn{5}{|c|}{ Men } & \multirow[b]{3}{*}{ TOTAL } \\
\hline & \multicolumn{3}{|c|}{ Age Group } & \multirow[b]{2}{*}{ TOTAL } & \multicolumn{4}{|c|}{ Age Group } & \multirow[b]{2}{*}{ TOTAL } & \\
\hline & 30 - 39 & $40-49$ & $50+$ & & $16-29$ & $30-39$ & $40-49$ & $50+$ & & \\
\hline Professional & 1 & 5 & 4 & 10 & 0 & 1 & 5 & 15 & 21 & 31 \\
\hline Administrative Support & 0 & 1 & 12 & 13 & 0 & 1 & 0 & 0 & 1 & 14 \\
\hline Technical Support & 0 & 2 & 0 & 2 & 0 & 0 & 0 & 1 & 1 & 3 \\
\hline Service & 0 & 3 & 0 & 3 & 1 & 3 & 2 & 10 & 16 & 19 \\
\hline Security and Fire & 0 & 0 & 0 & 0 & 0 & 1 & 3 & 0 & 4 & 4 \\
\hline Crafts & 0 & 1 & 0 & 1 & 0 & 0 & 2 & 11 & 13 & 14 \\
\hline Line Operators & 0 & 0 & 0 & 0 & 0 & 0 & 1 & 5 & 6 & 6 \\
\hline TOTAL & 1 & 12 & 16 & 29 & 1 & 6 & 13 & 42 & 62 & 91 \\
\hline
\end{tabular}

*Only those job categories and gender/age combinations with at least one absence appear in this table.

Appendix D. Total Number of Absences by Gender, Age, and Job Category*

\begin{tabular}{|c|c|c|c|c|c|c|c|c|c|c|}
\hline \multirow{3}{*}{ Job Category } & \multicolumn{4}{|c|}{ Women } & \multicolumn{5}{|c|}{ Men } & \multirow[b]{3}{*}{ TOTAL } \\
\hline & \multicolumn{3}{|c|}{ Age Group } & \multirow[b]{2}{*}{ TOTAL } & \multicolumn{4}{|c|}{ Age Group } & \multirow[b]{2}{*}{ TOTAL } & \\
\hline & $30-39$ & $40-49$ & $50+$ & & $16-29$ & $30-39$ & $40-49$ & $50+$ & & \\
\hline Professional & 1 & 5 & 4 & 10 & 0 & 1 & 5 & 17 & 23 & 33 \\
\hline Administrative Support & 0 & 1 & 14 & 15 & 0 & 1 & 0 & 0 & 1 & 16 \\
\hline Technical Support & 0 & 2 & 0 & 2 & 0 & 0 & 0 & 1 & 1 & 3 \\
\hline Service & 0 & 4 & 0 & 4 & 1 & 3 & 3 & 11 & 18 & 22 \\
\hline Security and Fire & 0 & 0 & 0 & 0 & 0 & 1 & 3 & 0 & 4 & 4 \\
\hline Crafts & 0 & 1 & 0 & 1 & 0 & 0 & 2 & 11 & 13 & 14 \\
\hline Line Operators & 0 & 0 & 0 & 0 & 0 & 0 & 1 & 5 & 6 & 6 \\
\hline TOTAL & 1 & 13 & 18 & 32 & 1 & 6 & 14 & 45 & 66 & 98 \\
\hline
\end{tabular}

*Only those job categories and gender/age combinations with at least one absence appear in this table. 
East Tennessee Technology Park 2008

Absence Data

Appendix E. Distribution of the Number of Calendar Days Missed per Absence by Gender and Age*

\begin{tabular}{|c|c|c|c|c|c|c|c|c|c|c|}
\hline \multirow{3}{*}{$\begin{array}{c}\text { Number of } \\
\text { Calendar Days }\end{array}$} & \multicolumn{4}{|c|}{ Women } & \multicolumn{5}{|c|}{ Men } & \multirow[b]{3}{*}{ TOTAL } \\
\hline & \multicolumn{3}{|c|}{ Age Group } & \multirow[b]{2}{*}{ TOTAL } & \multicolumn{4}{|c|}{ Age Group } & \multirow[b]{2}{*}{ TOTAL } & \\
\hline & $30-39$ & $40-49$ & $\mathbf{5 0 +}$ & & $16-29$ & 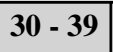 & $40-49$ & $50+$ & & \\
\hline$<15$ & 0 & 2 & 3 & 5 & 0 & 2 & 6 & 15 & 23 & 28 \\
\hline $15-28$ & 0 & 5 & 5 & 10 & 0 & 1 & 2 & 14 & 17 & 27 \\
\hline $29-42$ & 0 & 1 & 4 & 5 & 1 & 1 & 1 & 3 & 6 & 11 \\
\hline $43-56$ & 0 & 1 & 0 & 1 & 0 & 1 & 1 & 0 & 2 & 3 \\
\hline 57 - 91 & 0 & 3 & 1 & 4 & 0 & 0 & 2 & 6 & 8 & 12 \\
\hline $92-182$ & 1 & 1 & 3 & 5 & 0 & 1 & 2 & 7 & 10 & 15 \\
\hline $183+$ & 0 & 0 & 2 & 2 & 0 & 0 & 0 & 0 & 0 & 2 \\
\hline TOTAL & 1 & 13 & 18 & 32 & 1 & 6 & 14 & 45 & 66 & 98 \\
\hline
\end{tabular}

*Only those gender/age combinations with at least one absence appear in this table. 
East Tennessee Technology Park 2008

Absence Data

Appendix F. Distribution of the Number of Calendar Days Missed per Absence by Gender and Job Category*

Women

\begin{tabular}{|c|c|c|c|c|c|c|c|c|}
\hline \multirow{2}{*}{$\begin{array}{c}\text { Number of } \\
\text { Calendar Days }\end{array}$} & \multicolumn{7}{|c|}{ Job Category } & \multirow[b]{2}{*}{ TOTAL } \\
\hline & Professional & $\begin{array}{c}\text { Administrative } \\
\text { Support }\end{array}$ & Technical Support & Service & Security and Fire & Crafts & Line Operators & \\
\hline$<15$ & 0 & 3 & 0 & 2 & 0 & 0 & 0 & 5 \\
\hline $15-28$ & 5 & 4 & 1 & 0 & 0 & 0 & 0 & 10 \\
\hline $57-91$ & 1 & 1 & 1 & 0 & 0 & 1 & 0 & 4 \\
\hline $92-182$ & 1 & 3 & 0 & 1 & 0 & 0 & 0 & 5 \\
\hline $183+$ & 1 & 1 & 0 & 0 & 0 & 0 & 0 & 2 \\
\hline TOTAL & 10 & 15 & 2 & 4 & 0 & 1 & 0 & 32 \\
\hline
\end{tabular}

Men

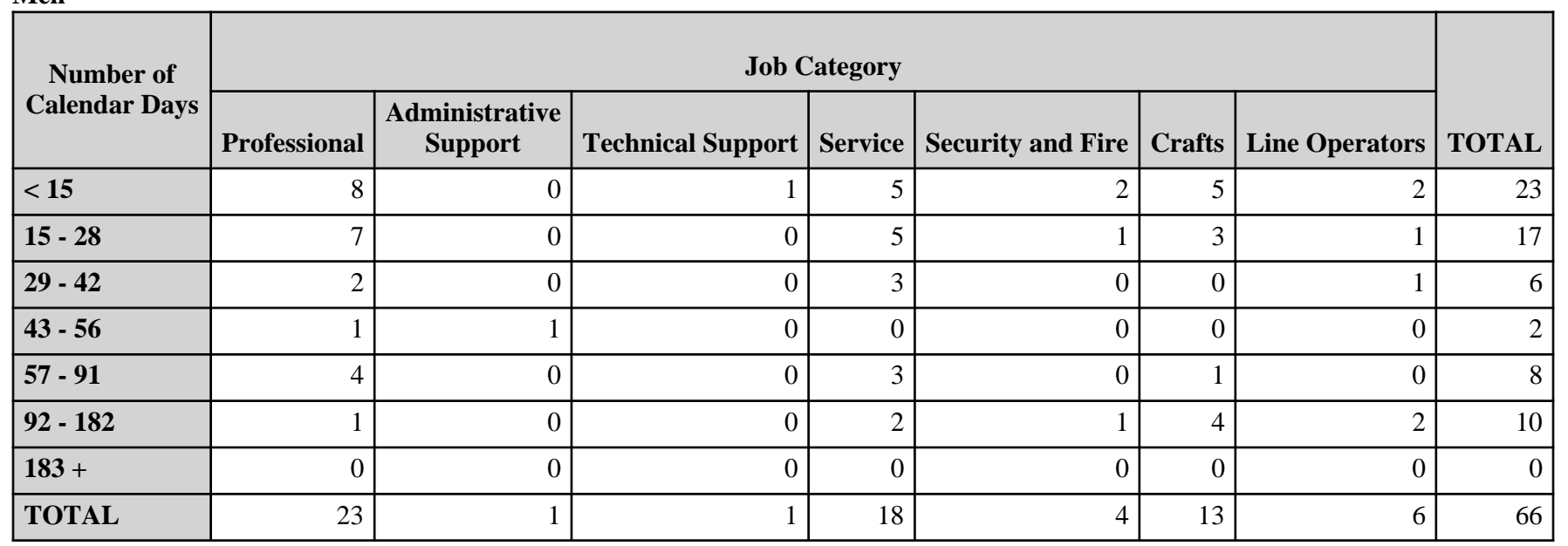

*Only those gender/job category combinations with at least one absence appear in this table. 
East Tennessee Technology Park 2008

Absence Data

Appendix G. Number of Diagnoses in Each Diagnostic Category by Gender and Age*

\begin{tabular}{|c|c|c|c|c|c|}
\hline & & \multicolumn{4}{|c|}{ Women } \\
\hline & & \multicolumn{3}{|c|}{ Age Group } & \multirow[b]{2}{*}{ TOTAL } \\
\hline & & $30-39$ & $40-49$ & $50+$ & \\
\hline Diagnostic Category & ICD-9-CM Code & \multirow[b]{2}{*}{0} & \multirow[b]{2}{*}{0} & \multirow[b]{2}{*}{0} & \multirow[b]{2}{*}{0} \\
\hline INFECTIOUS \& PARASITIC DISEASES (DIS) & \begin{tabular}{|c|}
$001-139$ \\
\end{tabular} & & & & \\
\hline -Other Bacterial Dis & $030-041$ & 0 & 0 & 0 & 0 \\
\hline MALIGNANT NEOPLASMS & $140-208,230-234$ & 0 & 0 & 0 & 0 \\
\hline -Lip, Oral Cavity, Pharynx & $140-149$ & 0 & 0 & 0 & 0 \\
\hline -Genitourinary & 179-189 & 0 & 0 & 0 & 0 \\
\hline -Lymphatic \& Hematopoietic & $200-208$ & 0 & 0 & 0 & 0 \\
\hline BENIGN \& UNCERTAIN NEOPLASMS & 210-229, 235-239 & 0 & 0 & 1 & 1 \\
\hline ENDOCRINE/METABOLIC/IMMUNITY & $240-279$ & 0 & 0 & 3 & 3 \\
\hline -Other Endocrine Gland Dis & $250-259$ & 0 & 0 & 1 & 1 \\
\hline -Other Metabolic \& Immunity Disorders & $270-279$ & 0 & 0 & 2 & 2 \\
\hline MENTAL DISORDERS & $290-319$ & 0 & 1 & 4 & 5 \\
\hline -Non-Psychotic Disorders & $300-302,306-316$ & 0 & 1 & 4 & 5 \\
\hline NERVOUS SYSTEM (NS) \& SENSE ORGANS & $320-389$ & 0 & 1 & 2 & 3 \\
\hline -Disorders of Peripheral NS & $350-359$ & 0 & 1 & 1 & 2 \\
\hline -Disorders of Eye & $360-379$ & 0 & 0 & 1 & 1 \\
\hline CIRCULATORY SYSTEM & $390-459$ & 0 & 1 & 3 & 4 \\
\hline -Hypertensive Dis & $401-405$ & 0 & 1 & 0 & 1 \\
\hline -Ischemic Heart Dis & $410-414$ & 0 & 0 & 1 & 1 \\
\hline -Dis of Pulmonary Circulation & $415-417$ & 0 & 0 & 0 & 0 \\
\hline -Other Heart Dis & $420-429$ & 0 & 0 & 0 & 0 \\
\hline -Cerebrovascular Dis & $430-438$ & 0 & 0 & 1 & 1 \\
\hline -Dis of Arteries \& Capillaries & $440-448$ & 0 & 0 & 1 & 1 \\
\hline -Dis of Veins, Lymphatics, Other & $451-459$ & 0 & 0 & 0 & 0 \\
\hline RESPIRATORY SYSTEM & $460-519$ & 0 & 2 & 1 & 3 \\
\hline -Acute Respiratory Infections & $460-466$ & 0 & 1 & 0 & 1 \\
\hline -Other Dis Upper Respiratory Tract & $470-478$ & 0 & 1 & 0 & 1 \\
\hline -Pneumonia \& Influenza & $480-487$ & 0 & 0 & 1 & 1 \\
\hline -Chronic Obstructive Dis & $490-496$ & 0 & 0 & 0 & 0 \\
\hline DIGESTIVE SYSTEM & $520-579$ & 0 & 0 & 4 & 4 \\
\hline -Esophagus, Stomach, Duodenum & $530-537$ & 0 & 0 & 1 & 1 \\
\hline -Hernia & $550-553$ & 0 & 0 & 1 & 1 \\
\hline -Other Intestinal Dis & $560-569$ & 0 & 0 & 0 & 0 \\
\hline -Other Digestive Dis & $570-579$ & 0 & 0 & 2 & 2 \\
\hline GENITOURINARY SYSTEM & $580-629$ & 0 & 3 & 3 & 6 \\
\hline -Other Urinary Dis & $590-599$ & 0 & 0 & 1 & 1 \\
\hline -Other Female Disorders & $617-629$ & 0 & 3 & 2 & 5 \\
\hline
\end{tabular}

(Continued)

*Only those diagnostic categories and gender/age combinations with at least one occurrence appear in this table. 
East Tennessee Technology Park 2008

Absence Data

Appendix G. Number of Diagnoses in Each Diagnostic Category by Gender and Age*

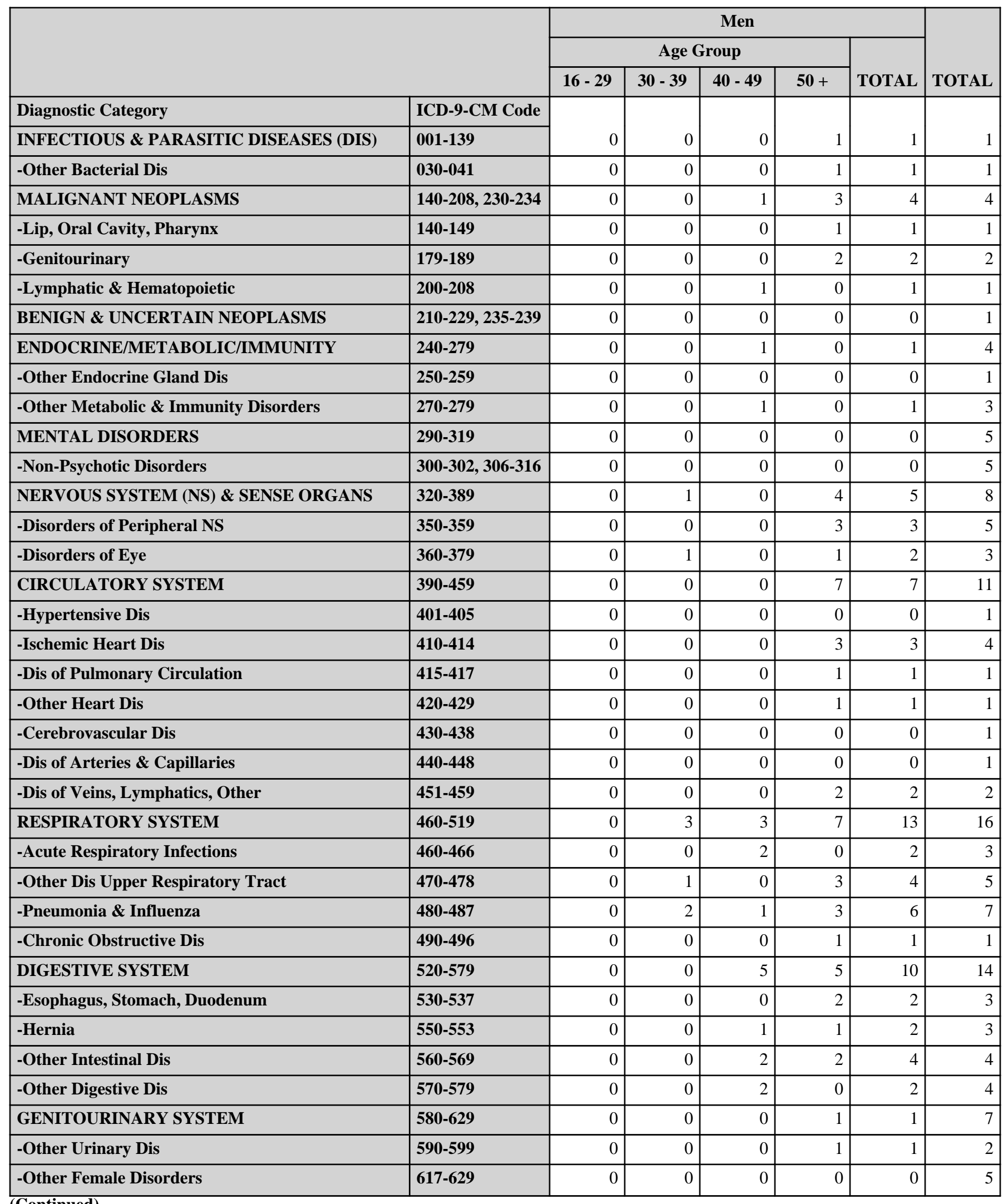

(Continued)

*Only those diagnostic categories and gender/age combinations with at least one occurrence appear in this table. 
East Tennessee Technology Park 2008

Absence Data

Appendix G. Number of Diagnoses in Each Diagnostic Category by Gender and Age*

\begin{tabular}{|c|c|c|c|c|c|}
\hline & & \multicolumn{4}{|c|}{ Women } \\
\hline & & \multicolumn{3}{|c|}{ Age Group } & \multirow[b]{2}{*}{ TOTAL } \\
\hline & & $30-39$ & $40-49$ & $50+$ & \\
\hline Diagnostic Category & ICD-9-CM Code & & & & \\
\hline $\begin{array}{l}\text { MUSCULOSKELETAL \& CONNECTIVE } \\
\text { TISSUE }\end{array}$ & $710-739$ & 1 & 6 & 4 & 11 \\
\hline -Arthropathies & $710-719$ & 0 & 0 & 3 & 3 \\
\hline -Dorsopathies & $720-724$ & 1 & 0 & 0 & 1 \\
\hline -Rheumatism, Excluding Back & $725-729$ & 0 & 3 & 1 & 4 \\
\hline -Other Dis \& Acquired Deformities & 730-739 & 0 & 3 & 0 & 3 \\
\hline CONGENITAL ANOMALIES & $740-759$ & 0 & 0 & 0 & 0 \\
\hline $\begin{array}{l}\text { SYMPTOMS, SIGNS, \& ILL-DEFINED } \\
\text { CONDITIONS }\end{array}$ & $780-799$ & 0 & 1 & 3 & 4 \\
\hline -Symptoms & 780-789 & 0 & 1 & 3 & $\overline{4}$ \\
\hline INJURY \& POISONING & $800-999$ & 0 & 3 & 1 & 4 \\
\hline -Fracture - Upper Limb & 810-819 & 0 & 0 & 0 & 0 \\
\hline -Fracture - Lower Limb & $820-829$ & 0 & 0 & 0 & 0 \\
\hline -Dislocation & 830-839 & 0 & 2 & 1 & 3 \\
\hline -Sprains \& Strains - Back & 846-847 & 0 & 0 & 0 & 0 \\
\hline -Sprains \& Strains - Other & $840-845,848$ & 0 & 1 & 0 & 1 \\
\hline -Open Wound - Upper Limb & 880-887 & 0 & 0 & 0 & 0 \\
\hline -Complications \& Unspecified Injuries & 958-959 & 0 & 0 & 0 & 0 \\
\hline -Unspecified Effects - External Causes & $990-995$ & 0 & 0 & 0 & 0 \\
\hline $\begin{array}{l}\text { HEALTH STATUS/HEALTH SERVICE } \\
\text { CONTACT }\end{array}$ & V01-V82 & 0 & 0 & 0 & 0 \\
\hline -Examination \& Investigation & V70-V82 & 0 & 0 & 0 & 0 \\
\hline
\end{tabular}

*Only those diagnostic categories and gender/age combinations with at least one occurrence appear in this table. 
East Tennessee Technology Park 2008

Absence Data

Appendix G. Number of Diagnoses in Each Diagnostic Category by Gender and Age*

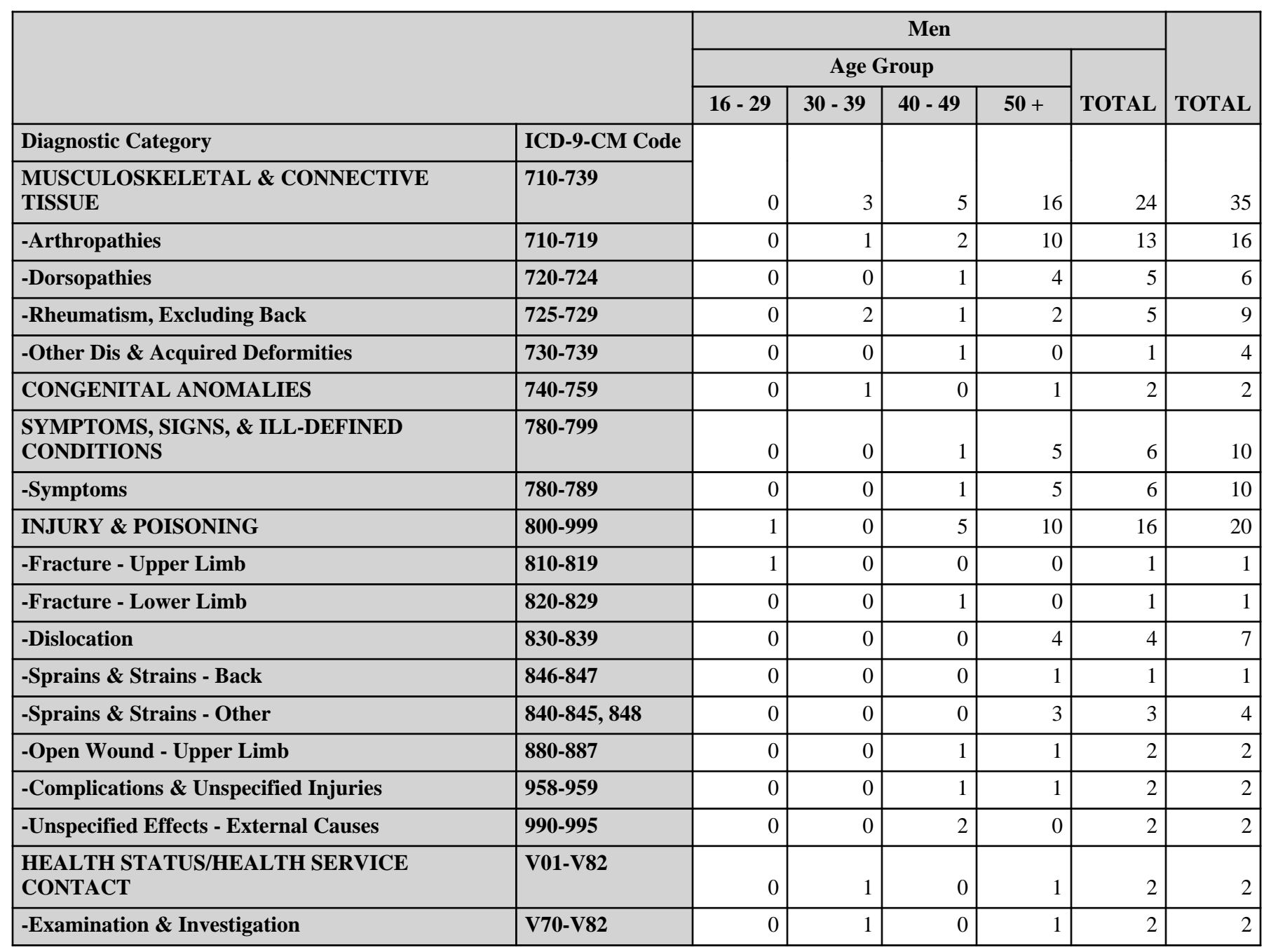

\begin{tabular}{|c|c|c|c|c|c|c|c|c|c|c|}
\hline & \multicolumn{4}{|c|}{ Women } & \multicolumn{5}{|c|}{ Men } & \multirow[b]{3}{*}{ TOTAL } \\
\hline & \multicolumn{3}{|c|}{ Age Group } & \multirow[b]{2}{*}{ TOTAL } & \multicolumn{4}{|c|}{ Age Group } & \multirow[b]{2}{*}{ TOTAL } & \\
\hline & $30-39$ & $40-49$ & $50+$ & & $16-29$ & $30-39$ & $40-49$ & $50+$ & & \\
\hline Diagnostic Category & \multirow[b]{2}{*}{1} & \multirow[b]{2}{*}{18} & \multirow[b]{2}{*}{29} & \multirow[b]{2}{*}{48} & \multirow[b]{2}{*}{1} & \multirow[b]{2}{*}{9} & \multirow[b]{2}{*}{21} & \multirow[b]{2}{*}{61} & \multirow[b]{2}{*}{92} & \multirow[b]{2}{*}{140} \\
\hline Total & & & & & & & & & & \\
\hline
\end{tabular}

*Only those diagnostic categories and gender/age combinations with at least one occurrence appear in this table. 
East Tennessee Technology Park 2008

Absence Data

Appendix H. Total Number of Calendar Days Absent in Each Diagnostic Category by Gender and Age*

\begin{tabular}{|c|c|c|c|c|c|}
\hline & & \multicolumn{4}{|c|}{ Women } \\
\hline & & \multicolumn{3}{|c|}{ Age Group } & \multirow[b]{2}{*}{ TOTAL } \\
\hline & & $30-39$ & $40-49$ & $50+$ & \\
\hline Diagnostic Category & ICD-9-CM Code & \multirow[b]{2}{*}{0} & \multirow[b]{2}{*}{0} & \multirow[b]{2}{*}{0} & \multirow[b]{2}{*}{0} \\
\hline INFECTIOUS \& PARASITIC DISEASES (DIS) & 001-139 & & & & \\
\hline MALIGNANT NEOPLASMS & $140-208,230-234$ & 0 & 0 & 0 & 0 \\
\hline BENIGN \& UNCERTAIN NEOPLASMS & 210-229, 235-239 & 0 & 0 & 13 & 13 \\
\hline ENDOCRINE/METABOLIC/IMMUNITY & $240-279$ & 0 & 0 & 391 & 391 \\
\hline MENTAL DISORDERS & $290-319$ & 0 & 110 & 205 & 315 \\
\hline NERVOUS SYSTEM (NS) \& SENSE ORGANS & 320-389 & 0 & 19 & 66 & 85 \\
\hline CIRCULATORY SYSTEM & $390-459$ & 0 & 5 & 11 & 16 \\
\hline RESPIRATORY SYSTEM & $460-519$ & 0 & 25 & 5 & 30 \\
\hline DIGESTIVE SYSTEM & $520-579$ & 0 & 0 & 98 & 98 \\
\hline GENITOURINARY SYSTEM & $580-629$ & 0 & 80 & 92 & 172 \\
\hline $\begin{array}{l}\text { MUSCULOSKELETAL \& CONNECTIVE } \\
\text { TISSUE }\end{array}$ & $710-739$ & 136 & 306 & 630 & 1,072 \\
\hline CONGENITAL ANOMALIES & $740-759$ & 0 & 0 & 0 & 0 \\
\hline $\begin{array}{l}\text { SYMPTOMS, SIGNS, \& ILL-DEFINED } \\
\text { CONDITIONS }\end{array}$ & $780-799$ & 0 & 5 & 55 & 60 \\
\hline INJURY \& POISONING & $800-999$ & 0 & 66 & 97 & 163 \\
\hline
\end{tabular}

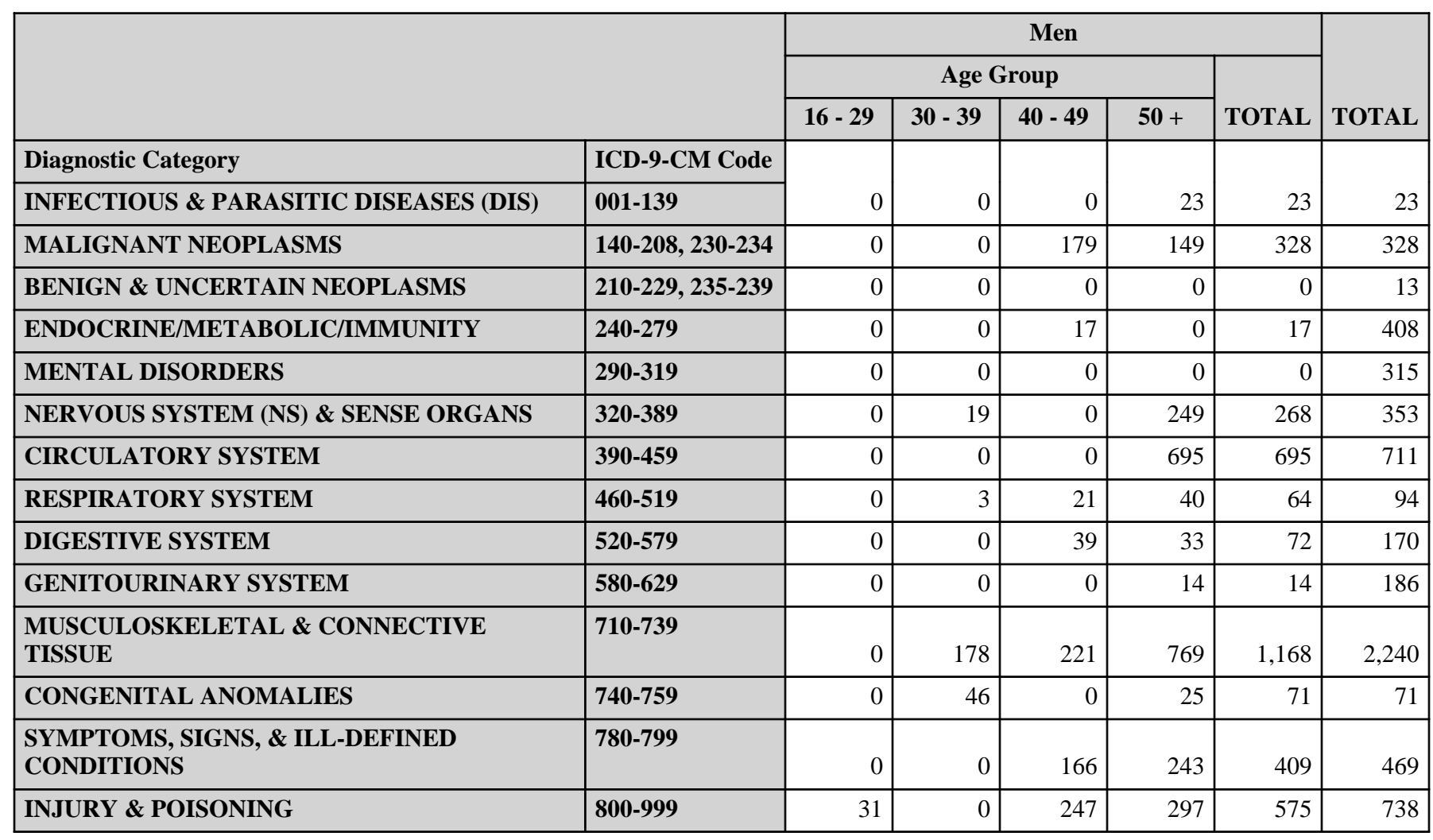

\footnotetext{
*Absences with >1 ICD-9-CM code in the same diagnostic category were counted only once. Only those diagnostic categories and gender/age combinations with at least one occurrence appear in this table.
} 
East Tennessee Technology Park 2008

Absence Data

Appendix I. Number of Diagnoses in Each Diagnostic Category by Gender and Job Category*

\begin{tabular}{|c|c|c|c|c|c|c|}
\hline & & \multicolumn{5}{|c|}{ Women } \\
\hline & & \multicolumn{5}{|c|}{ Job Category } \\
\hline & & Professional & $\begin{array}{l}\text { Administrative } \\
\text { Support }\end{array}$ & $\begin{array}{l}\text { Technical } \\
\text { Support }\end{array}$ & Service & Crafts \\
\hline Diagnostic Category & ICD-9-CM Code & \multirow[b]{2}{*}{0} & \multirow[b]{2}{*}{1} & \multirow[b]{2}{*}{0} & \multirow[b]{2}{*}{0} & \multirow[b]{2}{*}{0} \\
\hline BENIGN \& UNCERTAIN NEOPLASMS & 210-229, 235-239 & & & & & \\
\hline ENDOCRINE/METABOLIC/IMMUNITY & 240-279 & 2 & 1 & 0 & 0 & 0 \\
\hline -Other Endocrine Gland Dis & $250-259$ & 0 & 1 & 0 & 0 & 0 \\
\hline -Other Metabolic \& Immunity Disorders & 270-279 & 2 & 0 & 0 & 0 & 0 \\
\hline MENTAL DISORDERS & 290-319 & 1 & 3 & 0 & 1 & 0 \\
\hline -Non-Psychotic Disorders & $300-302,306-316$ & 1 & 3 & 0 & 1 & 0 \\
\hline NERVOUS SYSTEM (NS) \& SENSE ORGANS & 320-389 & 0 & 3 & 0 & 0 & 0 \\
\hline -Disorders of Peripheral NS & $350-359$ & 0 & 2 & 0 & 0 & 0 \\
\hline -Disorders of Eye & $360-379$ & 0 & 1 & 0 & 0 & 0 \\
\hline CIRCULATORY SYSTEM & $390-459$ & 0 & 3 & 0 & 1 & 0 \\
\hline -Hypertensive Dis & 401-405 & 0 & 0 & 0 & 1 & 0 \\
\hline -Ischemic Heart Dis & $410-414$ & 0 & 1 & 0 & 0 & 0 \\
\hline -Cerebrovascular Dis & $430-438$ & 0 & 1 & 0 & 0 & 0 \\
\hline -Dis of Arteries \& Capillaries & $440-448$ & 0 & 1 & 0 & 0 & 0 \\
\hline RESPIRATORY SYSTEM & $460-519$ & 1 & 1 & 0 & 1 & 0 \\
\hline -Acute Respiratory Infections & $460-466$ & 0 & 0 & 0 & 1 & 0 \\
\hline -Other Dis Upper Respiratory Tract & $470-478$ & 1 & 0 & 0 & 0 & 0 \\
\hline -Pneumonia \& Influenza & $480-487$ & 0 & 1 & 0 & 0 & 0 \\
\hline DIGESTIVE SYSTEM & $520-579$ & 2 & 2 & 0 & 0 & 0 \\
\hline -Esophagus, Stomach, Duodenum & $530-537$ & 1 & 0 & 0 & 0 & 0 \\
\hline -Hernia & $550-553$ & 0 & 1 & 0 & 0 & 0 \\
\hline -Other Digestive Dis & $570-579$ & 1 & 1 & 0 & 0 & 0 \\
\hline GENITOURINARY SYSTEM & $580-629$ & 2 & 3 & 0 & 1 & 0 \\
\hline -Other Urinary Dis & $590-599$ & 0 & 1 & 0 & 0 & 0 \\
\hline -Other Female Disorders & 617-629 & 2 & 2 & 0 & 1 & 0 \\
\hline $\begin{array}{l}\text { MUSCULOSKELETAL \& CONNECTIVE } \\
\text { TISSUE }\end{array}$ & $710-739$ & 5 & 3 & 2 & 0 & 1 \\
\hline -Arthropathies & $710-719$ & 0 & 3 & 0 & 0 & 0 \\
\hline -Dorsopathies & $720-724$ & 1 & 0 & 0 & 0 & 0 \\
\hline -Rheumatism, Excluding Back & 725-729 & 2 & 0 & 1 & 0 & 1 \\
\hline -Other Dis \& Acquired Deformities & $730-739$ & 2 & 0 & 1 & 0 & 0 \\
\hline $\begin{array}{l}\text { SYMPTOMS, SIGNS, \& ILL-DEFINED } \\
\text { CONDITIONS }\end{array}$ & $780-799$ & 2 & 1 & 0 & 1 & 0 \\
\hline -Symptoms & $780-789$ & 2 & 1 & 0 & 1 & 0 \\
\hline INJURY \& POISONING & 800-999 & 1 & 1 & 2 & 0 & 0 \\
\hline -Dislocation & 830-839 & 0 & 1 & 2 & 0 & 0 \\
\hline -Sprains \& Strains - Other & $840-845,848$ & 1 & 0 & 0 & 0 & 0 \\
\hline
\end{tabular}

*Only those diagnostic categories and gender/job category combinations with at least one occurrence appear in this table. 
East Tennessee Technology Park 2008

Absence Data

Appendix I. Number of Diagnoses in Each Diagnostic Category by Gender and Job Category*

\begin{tabular}{|c|c|c|}
\hline & & Women \\
\hline & & TOTAL \\
\hline Diagnostic Category & ICD-9-CM Code & \\
\hline BENIGN \& UNCERTAIN NEOPLASMS & $210-229,235-239$ & 1 \\
\hline ENDOCRINE/METABOLIC/IMMUNITY & $240-279$ & 3 \\
\hline -Other Endocrine Gland Dis & $250-259$ & 1 \\
\hline -Other Metabolic \& Immunity Disorders & $270-279$ & 2 \\
\hline MENTAL DISORDERS & 290-319 & 5 \\
\hline -Non-Psychotic Disorders & $300-302,306-316$ & 5 \\
\hline NERVOUS SYSTEM (NS) \& SENSE ORGANS & $320-389$ & 3 \\
\hline -Disorders of Peripheral NS & $350-359$ & 2 \\
\hline -Disorders of Eye & $360-379$ & 1 \\
\hline CIRCULATORY SYSTEM & $390-459$ & 4 \\
\hline -Hypertensive Dis & $401-405$ & 1 \\
\hline -Ischemic Heart Dis & $410-414$ & 1 \\
\hline -Cerebrovascular Dis & $430-438$ & 1 \\
\hline -Dis of Arteries \& Capillaries & $440-448$ & 1 \\
\hline RESPIRATORY SYSTEM & $460-519$ & 3 \\
\hline -Acute Respiratory Infections & $460-466$ & 1 \\
\hline -Other Dis Upper Respiratory Tract & $470-478$ & 1 \\
\hline -Pneumonia \& Influenza & $480-487$ & 1 \\
\hline DIGESTIVE SYSTEM & $520-579$ & 4 \\
\hline -Esophagus, Stomach, Duodenum & $\mathbf{5 3 0 - 5 3 7}$ & 1 \\
\hline -Hernia & $550-553$ & 1 \\
\hline -Other Digestive Dis & $570-579$ & 2 \\
\hline GENITOURINARY SYSTEM & $580-629$ & 6 \\
\hline -Other Urinary Dis & $590-599$ & 1 \\
\hline -Other Female Disorders & $617-629$ & 5 \\
\hline $\begin{array}{l}\text { MUSCULOSKELETAL \& CONNECTIVE } \\
\text { TISSUE }\end{array}$ & 710-739 & 11 \\
\hline -Arthropathies & 710-719 & 3 \\
\hline -Dorsopathies & $720-724$ & 1 \\
\hline -Rheumatism, Excluding Back & $725-729$ & 4 \\
\hline -Other Dis \& Acquired Deformities & \begin{tabular}{|l|l|}
$730-739$ \\
\end{tabular} & 3 \\
\hline $\begin{array}{l}\text { SYMPTOMS, SIGNS, \& ILL-DEFINED } \\
\text { CONDITIONS }\end{array}$ & 780-799 & 4 \\
\hline -Symptoms & $780-789$ & 4 \\
\hline INJURY \& POISONING & $800-999$ & 4 \\
\hline -Dislocation & 830-839 & 3 \\
\hline -Sprains \& Strains - Other & $840-845,848$ & 1 \\
\hline
\end{tabular}

*Only those diagnostic categories and gender/job category combinations with at least one occurrence appear in this table. 
East Tennessee Technology Park 2008

Absence Data

Appendix I. Number of Diagnoses in Each Diagnostic Category by Gender and Job Category*

\begin{tabular}{|c|c|c|c|c|c|c|}
\hline & \multicolumn{6}{|c|}{ Women } \\
\hline & \multicolumn{5}{|c|}{ Job Category } & \multirow[b]{2}{*}{ TOTAL } \\
\hline & Professional & $\begin{array}{c}\text { Administrative } \\
\text { Support }\end{array}$ & Technical Support & Service & Crafts & \\
\hline Diagnostic Category & \multirow[b]{2}{*}{16} & \multirow[b]{2}{*}{22} & \multirow[b]{2}{*}{4} & \multirow[b]{2}{*}{5} & \multirow[b]{2}{*}{1} & \multirow[b]{2}{*}{48} \\
\hline Total & & & & & & \\
\hline
\end{tabular}

*Only those diagnostic categories and gender/job category combinations with at least one occurrence appear in this table. 
East Tennessee Technology Park 2008

Absence Data

Appendix I. Number of Diagnoses in Each Diagnostic Category by Gender and Job Category*

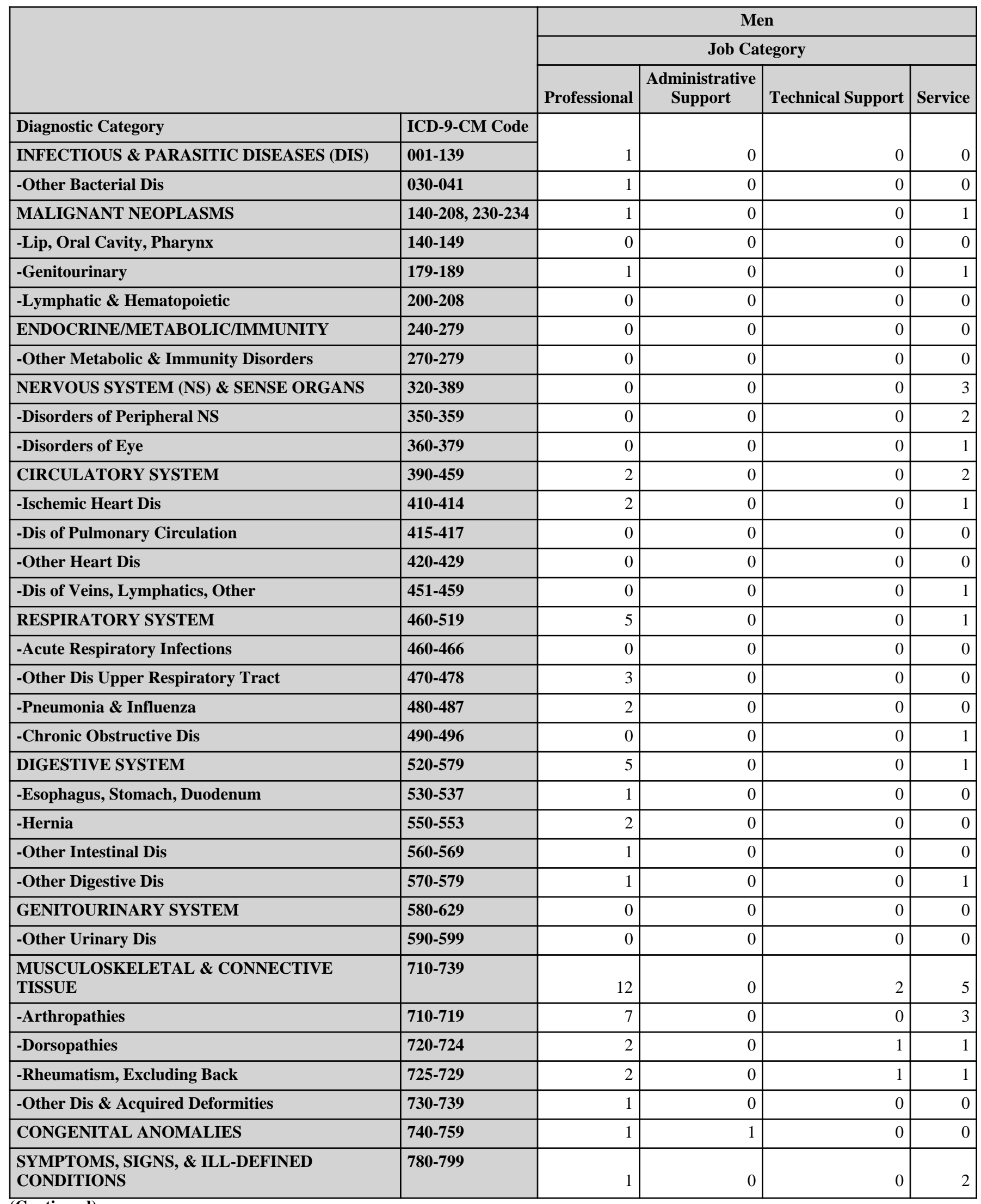

(Continued)

*Only those diagnostic categories and gender/job category combinations with at least one occurrence appear in this table. 
East Tennessee Technology Park 2008

Absence Data

Appendix I. Number of Diagnoses in Each Diagnostic Category by Gender and Job Category*

\begin{tabular}{|c|c|c|c|c|c|}
\hline & & \multicolumn{4}{|c|}{ Men } \\
\hline & & \multicolumn{3}{|c|}{ Job Category } & \multirow[b]{2}{*}{ TOTAL } \\
\hline & & Security and Fire & Crafts & Line Operators & \\
\hline Diagnostic Category & ICD-9-CM Code & \multirow[b]{2}{*}{0} & \multirow[b]{2}{*}{0} & \multirow[b]{2}{*}{0} & \multirow[b]{2}{*}{1} \\
\hline INFECTIOUS \& PARASITIC DISEASES (DIS) & 001-139 & & & & \\
\hline -Other Bacterial Dis & 030-041 & 0 & 0 & 0 & 1 \\
\hline MALIGNANT NEOPLASMS & $140-208,230-234$ & 1 & 1 & 0 & 4 \\
\hline -Lip, Oral Cavity, Pharynx & $140-149$ & 0 & 1 & 0 & 1 \\
\hline -Genitourinary & 179-189 & 0 & 0 & 0 & 2 \\
\hline -Lymphatic \& Hematopoietic & $200-208$ & 1 & 0 & 0 & 1 \\
\hline ENDOCRINE/METABOLIC/IMMUNITY & $240-279$ & 0 & 1 & 0 & 1 \\
\hline -Other Metabolic \& Immunity Disorders & $270-279$ & 0 & 1 & 0 & 1 \\
\hline NERVOUS SYSTEM (NS) \& SENSE ORGANS & 320-389 & 0 & 1 & 1 & 5 \\
\hline -Disorders of Peripheral NS & $350-359$ & 0 & 0 & 1 & 3 \\
\hline -Disorders of Eye & $360-379$ & 0 & 1 & 0 & 2 \\
\hline CIRCULATORY SYSTEM & $390-459$ & 0 & 2 & 1 & 7 \\
\hline -Ischemic Heart Dis & $410-414$ & 0 & 0 & 0 & 3 \\
\hline -Dis of Pulmonary Circulation & $415-417$ & 0 & 0 & 1 & 1 \\
\hline -Other Heart Dis & $420-429$ & 0 & 1 & 0 & 1 \\
\hline -Dis of Veins, Lymphatics, Other & 451-459 & 0 & 1 & 0 & 2 \\
\hline RESPIRATORY SYSTEM & $460-519$ & 4 & 1 & 2 & 13 \\
\hline -Acute Respiratory Infections & $460-466$ & 0 & 0 & 2 & 2 \\
\hline -Other Dis Upper Respiratory Tract & $470-478$ & 1 & 0 & 0 & 4 \\
\hline -Pneumonia \& Influenza & $480-487$ & 3 & 1 & 0 & 6 \\
\hline -Chronic Obstructive Dis & $490-496$ & 0 & 0 & 0 & 1 \\
\hline DIGESTIVE SYSTEM & $520-579$ & 2 & 0 & 2 & 10 \\
\hline -Esophagus, Stomach, Duodenum & $530-537$ & 0 & 0 & 1 & 2 \\
\hline -Hernia & $550-553$ & 0 & 0 & 0 & 2 \\
\hline -Other Intestinal Dis & $560-569$ & 2 & 0 & 1 & 4 \\
\hline -Other Digestive Dis & $570-579$ & 0 & 0 & 0 & 2 \\
\hline GENITOURINARY SYSTEM & $580-629$ & 0 & 1 & 0 & 1 \\
\hline -Other Urinary Dis & $590-599$ & 0 & 1 & 0 & 1 \\
\hline $\begin{array}{l}\text { MUSCULOSKELETAL \& CONNECTIVE } \\
\text { TISSUE }\end{array}$ & \begin{tabular}{|l|}
$710-739$ \\
\end{tabular} & 0 & 4 & 1 & 24 \\
\hline -Arthropathies & $710-719$ & 0 & 2 & 1 & 13 \\
\hline -Dorsopathies & $720-724$ & 0 & 1 & 0 & 5 \\
\hline -Rheumatism, Excluding Back & 725-729 & 0 & 1 & 0 & 5 \\
\hline -Other Dis \& Acquired Deformities & 730-739 & 0 & 0 & 0 & 1 \\
\hline CONGENITAL ANOMALIES & $740-759$ & 0 & 0 & 0 & 2 \\
\hline $\begin{array}{l}\text { SYMPTOMS, SIGNS, \& ILL-DEFINED } \\
\text { CONDITIONS }\end{array}$ & \begin{tabular}{|l|}
$780-799$ \\
\end{tabular} & 0 & 2 & 1 & 6 \\
\hline
\end{tabular}

(Continued)

*Only those diagnostic categories and gender/job category combinations with at least one occurrence appear in this table. 
East Tennessee Technology Park 2008

Absence Data

Appendix I. Number of Diagnoses in Each Diagnostic Category by Gender and Job Category*

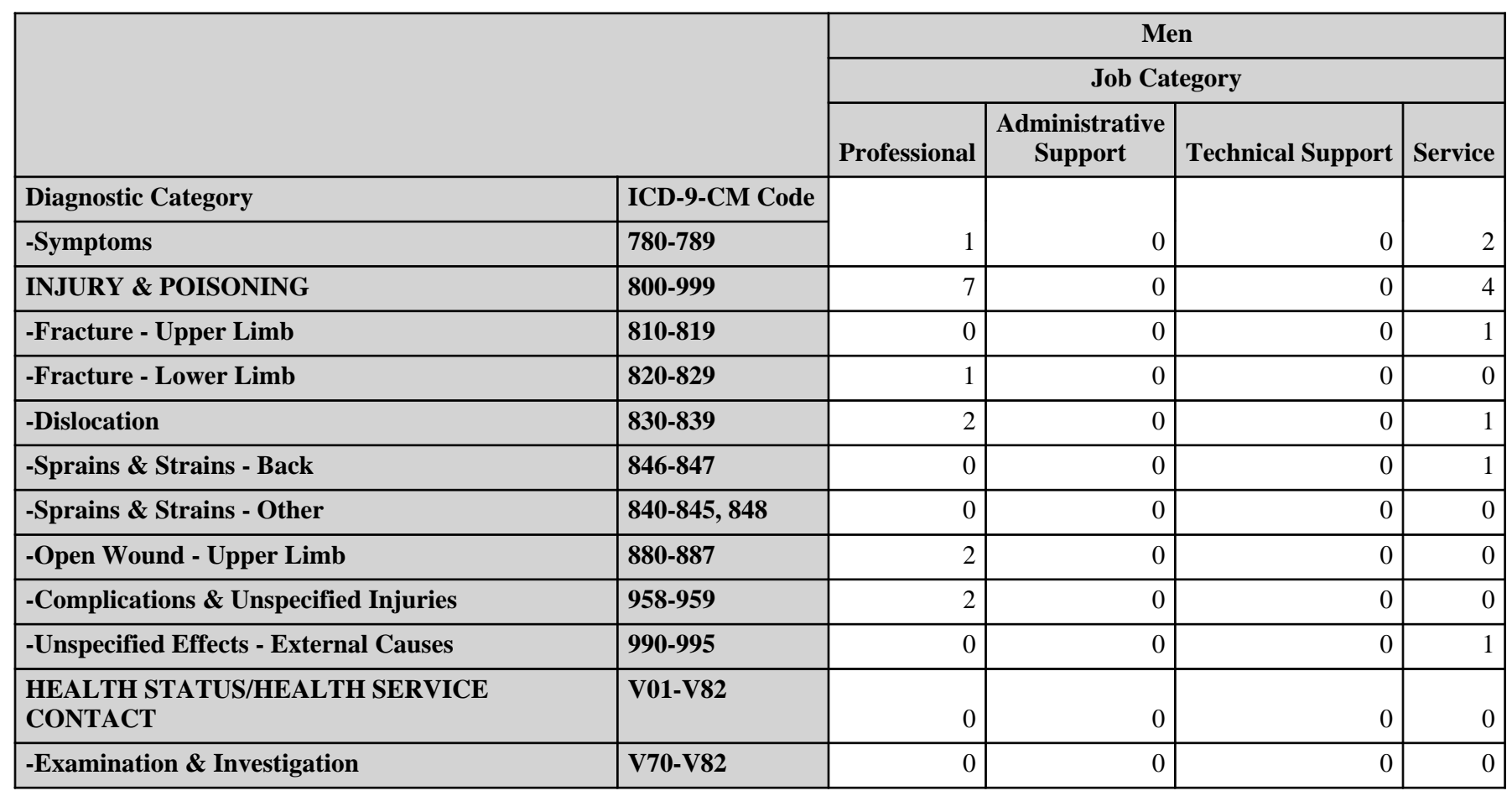

*Only those diagnostic categories and gender/job category combinations with at least one occurrence appear in this table. 
East Tennessee Technology Park 2008

Absence Data

Appendix I. Number of Diagnoses in Each Diagnostic Category by Gender and Job Category*

\begin{tabular}{|c|c|c|c|c|c|}
\hline & & \multicolumn{4}{|c|}{ Men } \\
\hline & & \multicolumn{3}{|c|}{ Job Category } & \multirow[b]{2}{*}{ TOTAL } \\
\hline & & Security and Fire & Crafts & Line Operators & \\
\hline Diagnostic Category & ICD-9-CM Code & \multirow[b]{2}{*}{0} & \multirow[b]{2}{*}{2} & \multirow[b]{2}{*}{1} & \multirow[b]{2}{*}{6} \\
\hline -Symptoms & 780-789 & & & & \\
\hline INJURY \& POISONING & $800-999$ & 0 & 2 & 3 & 16 \\
\hline -Fracture - Upper Limb & $810-819$ & 0 & 0 & 0 & 1 \\
\hline -Fracture - Lower Limb & $820-829$ & 0 & 0 & 0 & 1 \\
\hline -Dislocation & 830-839 & 0 & 1 & 0 & 4 \\
\hline -Sprains \& Strains - Back & 846-847 & 0 & 0 & 0 & 1 \\
\hline -Sprains \& Strains - Other & $840-845,848$ & 0 & 0 & 3 & 3 \\
\hline -Open Wound - Upper Limb & $880-887$ & 0 & 0 & 0 & 2 \\
\hline -Complications \& Unspecified Injuries & 958-959 & 0 & 0 & 0 & 2 \\
\hline -Unspecified Effects - External Causes & $990-995$ & 0 & 1 & 0 & 2 \\
\hline $\begin{array}{l}\text { HEALTH STATUS/HEALTH SERVICE } \\
\text { CONTACT }\end{array}$ & V01-V82 & 0 & 0 & 2 & 2 \\
\hline -Examination \& Investigation & V70-V82 & 0 & 0 & 2 & 2 \\
\hline
\end{tabular}

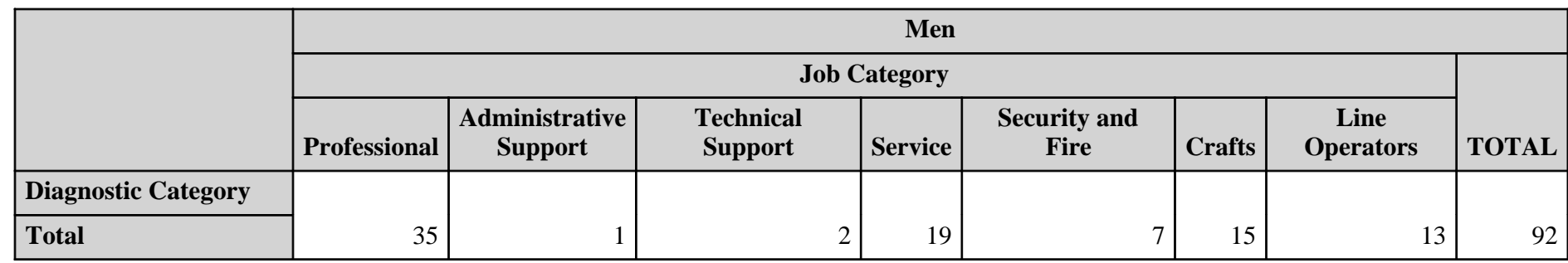

*Only those diagnostic categories and gender/job category combinations with at least one occurrence appear in this table. 
East Tennessee Technology Park 2008

Absence Data

Appendix J. Total Number of Calendar Days Absent in Each Diagnostic Category by Gender and Job Category*

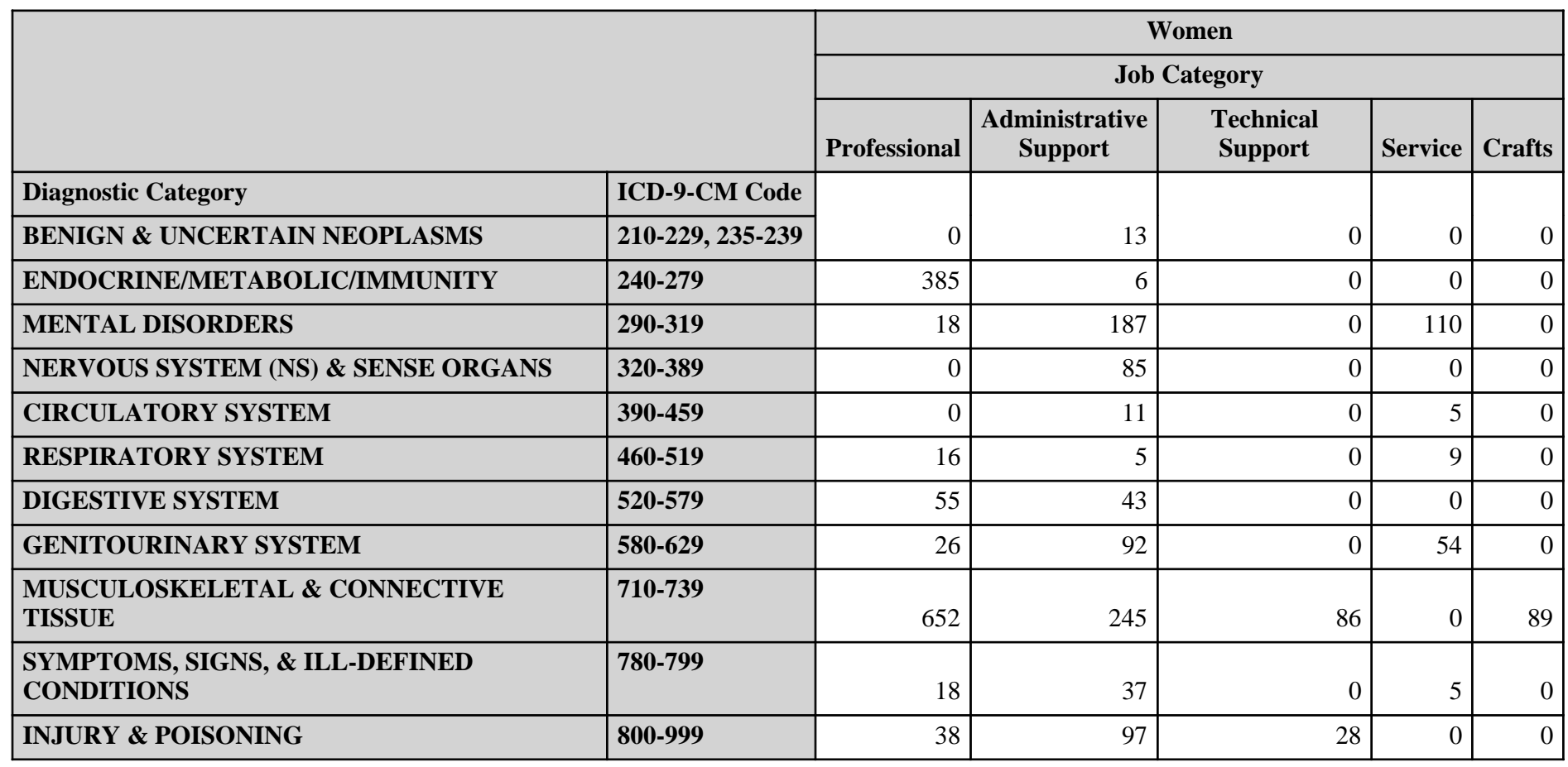

\begin{tabular}{|c|c|c|}
\hline & & Women \\
\hline & & TOTAL \\
\hline Diagnostic Category & ICD-9-CM Code & \\
\hline BENIGN \& UNCERTAIN NEOPLASMS & 210-229, 235-239 & 13 \\
\hline ENDOCRINE/METABOLIC/IMMUNITY & 240-279 & 391 \\
\hline MENTAL DISORDERS & $290-319$ & 315 \\
\hline NERVOUS SYSTEM (NS) \& SENSE ORGANS & 320-389 & 85 \\
\hline CIRCULATORY SYSTEM & $390-459$ & 16 \\
\hline RESPIRATORY SYSTEM & $460-519$ & 30 \\
\hline DIGESTIVE SYSTEM & 520-579 & 98 \\
\hline GENITOURINARY SYSTEM & $580-629$ & 172 \\
\hline $\begin{array}{l}\text { MUSCULOSKELETAL \& CONNECTIVE } \\
\text { TISSUE }\end{array}$ & 710-739 & 1,072 \\
\hline $\begin{array}{l}\text { SYMPTOMS, SIGNS, \& ILL-DEFINED } \\
\text { CONDITIONS }\end{array}$ & 780-799 & 60 \\
\hline INJURY \& POISONING & $800-999$ & 163 \\
\hline
\end{tabular}

\footnotetext{
*Absences with >1 ICD-9-CM code in the same diagnostic category were counted only once. Only those diagnostic categories and gender/job category combinations with at least one occurrence appear in this table.
} 
East Tennessee Technology Park 2008

Absence Data

Appendix J. Total Number of Calendar Days Absent in Each Diagnostic Category by Gender and Job Category*

\begin{tabular}{|c|c|c|c|c|c|}
\hline & \multicolumn{4}{|c|}{ Men } \\
\hline & & \multicolumn{4}{|c|}{ Job Category } \\
\hline & & Professional & $\begin{array}{c}\text { Administrative } \\
\text { Support }\end{array}$ & Technical Support & Service \\
\hline Diagnostic Category & ICD-9-CM Code & \multirow[b]{2}{*}{23} & \multirow[b]{2}{*}{0} & \multirow[b]{2}{*}{0} & \multirow[b]{2}{*}{0} \\
\hline INFECTIOUS \& PARASITIC DISEASES (DIS) & 001-139 & & & & \\
\hline MALIGNANT NEOPLASMS & $140-208,230-234$ & 66 & 0 & 0 & 69 \\
\hline ENDOCRINE/METABOLIC/IMMUNITY & $240-279$ & 0 & 0 & 0 & 0 \\
\hline NERVOUS SYSTEM (NS) \& SENSE ORGANS & $320-389$ & 0 & 0 & 0 & 69 \\
\hline CIRCULATORY SYSTEM & $390-459$ & 146 & 0 & 0 & 191 \\
\hline RESPIRATORY SYSTEM & $460-519$ & 32 & 0 & 0 & 1 \\
\hline DIGESTIVE SYSTEM & $520-579$ & 41 & 0 & 0 & 12 \\
\hline GENITOURINARY SYSTEM & 580-629 & 0 & 0 & 0 & 0 \\
\hline $\begin{array}{l}\text { MUSCULOSKELETAL \& CONNECTIVE } \\
\text { TISSUE }\end{array}$ & 710-739 & 373 & 0 & 6 & 361 \\
\hline CONGENITAL ANOMALIES & 740-759 & 25 & 46 & 0 & 0 \\
\hline $\begin{array}{l}\text { SYMPTOMS, SIGNS, \& ILL-DEFINED } \\
\text { CONDITIONS }\end{array}$ & 780-799 & 28 & 0 & 0 & 37 \\
\hline INJURY \& POISONING & $800-999$ & 149 & 0 & 0 & 137 \\
\hline
\end{tabular}

\begin{tabular}{|c|c|c|c|c|c|}
\hline & & \multicolumn{4}{|c|}{ Men } \\
\hline & & \multicolumn{3}{|c|}{ Job Category } & \multirow[b]{2}{*}{ TOTAL } \\
\hline & & Security and Fire & Crafts & Line Operators & \\
\hline Diagnostic Category & ICD-9-CM Code & \multirow[b]{2}{*}{0} & \multirow[b]{2}{*}{0} & \multirow[b]{2}{*}{0} & \multirow[b]{2}{*}{23} \\
\hline INFECTIOUS \& PARASITIC DISEASES (DIS) & $001-139$ & & & & \\
\hline MALIGNANT NEOPLASMS & $140-208,230-234$ & 179 & 14 & 0 & 328 \\
\hline ENDOCRINE/METABOLIC/IMMUNITY & $240-279$ & 0 & 17 & 0 & 17 \\
\hline NERVOUS SYSTEM (NS) \& SENSE ORGANS & $320-389$ & 0 & 178 & 21 & 268 \\
\hline CIRCULATORY SYSTEM & $390-459$ & 0 & 192 & 166 & 695 \\
\hline RESPIRATORY SYSTEM & $460-519$ & 14 & 7 & 10 & 64 \\
\hline DIGESTIVE SYSTEM & $520-579$ & 15 & 0 & 4 & 72 \\
\hline GENITOURINARY SYSTEM & $580-629$ & 0 & 14 & 0 & 14 \\
\hline $\begin{array}{l}\text { MUSCULOSKELETAL \& CONNECTIVE } \\
\text { TISSUE }\end{array}$ & 710-739 & 0 & 284 & 144 & 1,168 \\
\hline CONGENITAL ANOMALIES & $740-759$ & 0 & 0 & 0 & 71 \\
\hline $\begin{array}{l}\text { SYMPTOMS, SIGNS, \& ILL-DEFINED } \\
\text { CONDITIONS }\end{array}$ & 780-799 & 0 & 178 & 166 & 409 \\
\hline INJURY \& POISONING & $800-999$ & 0 & 229 & 60 & 575 \\
\hline
\end{tabular}

\footnotetext{
*Absences with >1 ICD-9-CM code in the same diagnostic category were counted only once. Only those diagnostic categories and gender/job category combinations with at least one occurrence appear in this table.
} 
East Tennessee Technology Park 2008

Absence Data

Appendix K. Age-Adjusted IIIness and Injury Rates by Diagnostic Category*

Part 1. Men

\begin{tabular}{|c|c|c|c|c|c|}
\hline & & $\begin{array}{l}\text { Number of } \\
\text { Diagnoses }\end{array}$ & $\begin{array}{c}\text { Age-Adjusted } \\
\text { Rate per } 1,000 * *\end{array}$ & $\begin{array}{c}\text { Lower } 95 \% \\
\text { Confidence } \\
\text { Limit per 1,000 }\end{array}$ & $\begin{array}{c}\text { Upper 95\% } \\
\text { Confidence } \\
\text { Limit per 1,000 }\end{array}$ \\
\hline Diagnostic Category & ICD-9-CM Code & & & & \\
\hline INFECTIOUS \& PARASITIC DISEASES (DIS) & 001-139 & 1 & 0.8 & 0.1 & 5.4 \\
\hline -Other Bacterial Dis & \begin{tabular}{|c|}
$030-041$ \\
\end{tabular} & 1 & 0.8 & 0.1 & 5.4 \\
\hline MALIGNANT NEOPLASMS & $140-208,230-234$ & 4 & 2.7 & 1.0 & 7.4 \\
\hline -Lip, Oral Cavity, Pharynx & $140-149$ & 1 & 0.8 & 0.1 & 5.4 \\
\hline -Genitourinary & 179-189 & 2 & 1.2 & 0.3 & 5.0 \\
\hline -Lymphatic \& Hematopoietic & $200-208$ & 1 & 0.8 & 0.1 & 5.5 \\
\hline ENDOCRINE/METABOLIC/IMMUNITY & $240-279$ & 1 & 0.8 & 0.1 & 5.5 \\
\hline -Other Metabolic \& Immunity Disorders & $270-279$ & 1 & 0.8 & 0.1 & 5.5 \\
\hline NERVOUS SYSTEM (NS) \& SENSE ORGANS & 320-389 & 5 & 4.1 & 1.6 & 10.6 \\
\hline -Disorders of Peripheral NS & $350-359$ & 3 & 2.0 & 0.6 & 6.3 \\
\hline -Disorders of Eye & $360-379$ & 2 & 2.2 & 0.5 & 9.3 \\
\hline CIRCULATORY SYSTEM & $390-459$ & 7 & 3.7 & 1.7 & 8.0 \\
\hline -Ischemic Heart Dis & $410-414$ & 3 & 1.6 & 0.5 & 5.3 \\
\hline -Dis of Pulmonary Circulation & $415-417$ & 1 & 0.4 & 0.1 & 3.1 \\
\hline -Other Heart Dis & $420-429$ & 1 & 0.8 & 0.1 & 5.4 \\
\hline -Dis of Veins, Lymphatics, Other & $451-459$ & 2 & 0.9 & 0.2 & 3.5 \\
\hline RESPIRATORY SYSTEM & $460-519$ & 13 & 10.0 & 5.4 & 18.4 \\
\hline -Acute Respiratory Infections & $460-466$ & 2 & 1.6 & 0.4 & 6.2 \\
\hline -Other Dis Upper Respiratory Tract & $470-478$ & 4 & 2.7 & 0.9 & 8.7 \\
\hline -Pneumonia \& Influenza & $480-487$ & 6 & 5.3 & 2.2 & 12.8 \\
\hline -Chronic Obstructive Dis & $490-496$ & 1 & 0.4 & 0.1 & 3.1 \\
\hline DIGESTIVE SYSTEM & $520-579$ & 10 & 6.4 & 3.4 & 12.1 \\
\hline -Esophagus, Stomach, Duodenum & $\mathbf{5 3 0 - 5 3 7}$ & 2 & 0.9 & 0.2 & 3.5 \\
\hline -Hernia & $550-553$ & 2 & 1.5 & 0.4 & 6.1 \\
\hline -Other Intestinal Dis & $560-569$ & 4 & 2.4 & 0.9 & 6.7 \\
\hline -Other Digestive Dis & \begin{tabular}{|l|}
$570-579$ \\
\end{tabular} & 2 & 1.6 & 0.4 & 6.2 \\
\hline GENITOURINARY SYSTEM & $580-629$ & 1 & 0.8 & 0.1 & 5.4 \\
\hline -Other Urinary Dis & $590-599$ & 1 & 0.8 & 0.1 & 5.4 \\
\hline $\begin{array}{l}\text { MUSCULOSKELETAL \& CONNECTIVE } \\
\text { TISSUE }\end{array}$ & $710-739$ & 24 & 16.8 & 10.8 & 26.1 \\
\hline -Arthropathies & $710-719$ & 13 & 8.3 & 4.6 & 15.1 \\
\hline -Dorsopathies & $720-724$ & 5 & 3.2 & 1.3 & 7.8 \\
\hline -Rheumatism, Excluding Back & 725-729 & 5 & 4.5 & 1.7 & 12.0 \\
\hline -Other Dis \& Acquired Deformities & 730-739 & 1 & 0.8 & 0.1 & 5.5 \\
\hline CONGENITAL ANOMALIES & $740-759$ & 2 & 1.9 & 0.4 & 9.0 \\
\hline
\end{tabular}

(Continued)

*Only those diagnostic categories with at least one occurrence appear in this table.

**Standardized to age distribution of 2000 U.S. population. 
East Tennessee Technology Park 2008

Absence Data

Appendix K. Age-Adjusted IIlness and Injury Rates by Diagnostic Category*

Part 1. Men

\begin{tabular}{|c|c|c|c|c|c|}
\hline & & $\begin{array}{l}\text { Number of } \\
\text { Diagnoses }\end{array}$ & $\begin{array}{c}\text { Age-Adjusted } \\
\text { Rate per } 1,000 * *\end{array}$ & $\begin{array}{c}\text { Lower } 95 \% \\
\text { Confidence } \\
\text { Limit per 1,000 }\end{array}$ & $\begin{array}{c}\text { Upper 95\% } \\
\text { Confidence } \\
\text { Limit per 1,000 }\end{array}$ \\
\hline Diagnostic Category & ICD-9-CM Code & & & & \\
\hline $\begin{array}{l}\text { SYMPTOMS, SIGNS, \& ILL-DEFINED } \\
\text { CONDITIONS }\end{array}$ & 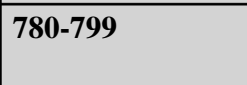 & 6 & 3.0 & 1.3 & 6.8 \\
\hline -Symptoms & $780-789$ & 6 & 3.0 & 1.3 & 6.8 \\
\hline INJURY \& POISONING & $800-999$ & 16 & 10.3 & 6.1 & 17.5 \\
\hline -Fracture - Upper Limb & $810-819$ & 1 & 1.4 & 0.2 & 10.2 \\
\hline -Fracture - Lower Limb & \begin{tabular}{|c|}
$820-829$ \\
\end{tabular} & 1 & 0.8 & 0.1 & 5.5 \\
\hline -Dislocation & $830-839$ & 4 & 2.1 & 0.8 & 5.7 \\
\hline -Sprains \& Strains - Back & 846-847 & 1 & 0.4 & 0.1 & 3.1 \\
\hline -Sprains \& Strains - Other & $840-845,848$ & 3 & 1.3 & 0.4 & 4.1 \\
\hline -Open Wound - Head, Neck, Trunk & $870-879$ & 2 & 1.5 & 0.4 & 6.1 \\
\hline -Complications \& Unspecified Injuries & \begin{tabular}{|c|}
$958-959$ \\
\end{tabular} & 2 & 1.2 & 0.3 & 5.1 \\
\hline -Unspecified Effects - External Causes & $990-995$ & 2 & 1.6 & 0.4 & 6.2 \\
\hline Total & & 90 & 61.3 & 48.9 & 76.8 \\
\hline
\end{tabular}

* Only those diagnostic categories with at least one occurrence appear in this table.

**Standardized to age distribution of 2000 U.S. population. 
East Tennessee Technology Park 2008

Absence Data

Appendix K. Age-Adjusted Illness and Injury Rates by Diagnostic Category*

Part 2. Women

\begin{tabular}{|c|c|c|c|c|c|}
\hline & & $\begin{array}{l}\text { Number of } \\
\text { Diagnoses }\end{array}$ & $\begin{array}{c}\text { Age-Adjusted } \\
\text { Rate per } 1,000 * *\end{array}$ & $\begin{array}{c}\text { Lower } 95 \% \\
\text { Confidence } \\
\text { Limit per 1,000 }\end{array}$ & $\begin{array}{c}\text { Upper } 95 \% \\
\text { Confidence } \\
\text { Limit per 1,000 }\end{array}$ \\
\hline Diagnostic Category & ICD-9-CM Code & \multirow[b]{2}{*}{1} & \multirow[b]{2}{*}{1.2} & \multirow[b]{2}{*}{0.2} & \multirow[b]{2}{*}{8.6} \\
\hline BENIGN \& UNCERTAIN NEOPLASMS & $210-229,235-239$ & & & & \\
\hline ENDOCRINE/METABOLIC/IMMUNITY & $240-279$ & 3 & 9.0 & 2.6 & 30.7 \\
\hline -Other Endocrine Gland Dis & $250-259$ & 1 & 1.2 & 0.2 & 8.6 \\
\hline -Other Metabolic \& Immunity Disorders & $270-279$ & 2 & 7.8 & 1.9 & 31.1 \\
\hline MENTAL DISORDERS & 290-319 & 5 & 6.6 & 2.7 & 15.9 \\
\hline -Non-Psychotic Disorders & $300-302,306-316$ & 5 & 6.6 & 2.7 & 15.9 \\
\hline NERVOUS SYSTEM (NS) \& SENSE ORGANS & $320-389$ & 3 & 4.1 & 1.3 & 13.1 \\
\hline -Disorders of Peripheral NS & $350-359$ & 2 & 2.9 & 0.7 & 12.0 \\
\hline -Disorders of Eye & $360-379$ & 1 & 1.2 & 0.2 & 8.6 \\
\hline CIRCULATORY SYSTEM & $390-459$ & 4 & 8.0 & 2.6 & 24.6 \\
\hline -Hypertensive Dis & $401-405$ & 1 & 1.7 & 0.2 & 12.2 \\
\hline -Ischemic Heart Dis & $410-414$ & 1 & 3.9 & 0.5 & 27.6 \\
\hline -Cerebrovascular Dis & $430-438$ & 1 & 1.2 & 0.2 & $\overline{8.6}$ \\
\hline -Dis of Arteries \& Capillaries & $440-448$ & 1 & 1.2 & 0.2 & 8.6 \\
\hline RESPIRATORY SYSTEM & $460-519$ & 3 & 7.3 & 2.1 & 25.0 \\
\hline -Acute Respiratory Infections & $460-466$ & 1 & 1.7 & 0.2 & 12.2 \\
\hline -Other Dis Upper Respiratory Tract & $470-478$ & 1 & 1.7 & 0.2 & 12.2 \\
\hline -Pneumonia \& Influenza & $480-487$ & 1 & 3.9 & 0.5 & 27.6 \\
\hline DIGESTIVE SYSTEM & $520-579$ & 4 & 4.8 & 1.8 & 12.9 \\
\hline -Esophagus, Stomach, Duodenum & $530-537$ & 1 & 1.2 & 0.2 & 8.6 \\
\hline -Hernia & $550-553$ & 1 & 1.2 & 0.2 & 8.6 \\
\hline -Other Digestive Dis & $570-579$ & 2 & 2.4 & 0.6 & 9.7 \\
\hline GENITOURINARY SYSTEM & $580-629$ & 6 & 8.8 & 3.9 & 19.8 \\
\hline -Other Urinary Dis & $590-599$ & 1 & 1.2 & 0.2 & 8.6 \\
\hline -Other Female Disorders & $617-629$ & 5 & 7.6 & 3.1 & 18.4 \\
\hline $\begin{array}{l}\text { MUSCULOSKELETAL \& CONNECTIVE } \\
\text { TISSUE }\end{array}$ & $710-739$ & 11 & 25.8 & 13.1 & 50.7 \\
\hline -Arthropathies & $710-719$ & 3 & 6.3 & 1.7 & 23.6 \\
\hline -Dorsopathies & $720-724$ & 1 & 5.3 & 0.7 & 37.6 \\
\hline -Rheumatism, Excluding Back & 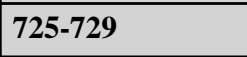 & 4 & 9.0 & 3.1 & 26.1 \\
\hline -Other Dis \& Acquired Deformities & 730-739 & 3 & 5.2 & 1.7 & 16.0 \\
\hline $\begin{array}{l}\text { SYMPTOMS, SIGNS, \& ILL-DEFINED } \\
\text { CONDITIONS }\end{array}$ & \begin{tabular}{|l|}
$780-799$ \\
\end{tabular} & 4 & 5.4 & 2.0 & 14.5 \\
\hline -Symptoms & $780-789$ & 4 & 5.4 & 2.0 & 14.5 \\
\hline INJURY \& POISONING & \begin{tabular}{|c|}
$800-999$ \\
\end{tabular} & 4 & 6.4 & 2.4 & 17.1 \\
\hline -Dislocation & $830-839$ & 3 & 4.7 & 1.5 & 14.6 \\
\hline
\end{tabular}

(Continued)

*Only those diagnostic categories with at least one occurrence appear in this table.

**Standardized to age distribution of 2000 U.S. population. 
East Tennessee Technology Park 2008

Absence Data

Appendix K. Age-Adjusted IIlness and Injury Rates by Diagnostic Category*

Part 2. Women

\begin{tabular}{|c|c|c|c|c|c|}
\hline & & $\begin{array}{l}\text { Number of } \\
\text { Diagnoses }\end{array}$ & $\begin{array}{c}\text { Age-Adjusted } \\
\text { Rate per } 1,000^{* * *}\end{array}$ & $\begin{array}{c}\text { Lower } 95 \% \\
\text { Confidence } \\
\text { Limit per 1,000 }\end{array}$ & $\begin{array}{c}\text { Upper 95\% } \\
\text { Confidence } \\
\text { Limit per 1,000 }\end{array}$ \\
\hline Diagnostic Category & ICD-9-CM Code & \multirow[b]{2}{*}{1} & \multirow[b]{2}{*}{1.7} & \multirow[b]{2}{*}{0.2} & \multirow[b]{2}{*}{12.2} \\
\hline -Sprains \& Strains - Other & $840-845,848$ & & & & \\
\hline Total & & 48 & 87.4 & 63.4 & 120.5 \\
\hline
\end{tabular}

* Only those diagnostic categories with at least one occurrence appear in this table.

**Standardized to age distribution of 2000 U.S. population. 
East Tennessee Technology Park 2008

Absence Data

Appendix K. Age-Adjusted Illness and Injury Rates by Diagnostic Category*

Part 3. Men and Women

\begin{tabular}{|c|c|c|c|c|c|}
\hline & & $\begin{array}{l}\text { Number of } \\
\text { Diagnoses }\end{array}$ & $\begin{array}{c}\text { Age-Adjusted } \\
\text { Rate per } 1,000 * *\end{array}$ & $\begin{array}{c}\text { Lower } 95 \% \\
\text { Confidence } \\
\text { Limit per 1,000 }\end{array}$ & $\begin{array}{c}\text { Upper } 95 \% \\
\text { Confidence } \\
\text { Limit per 1,000 }\end{array}$ \\
\hline Diagnostic Category & ICD-9-CM Code & & & & \\
\hline INFECTIOUS \& PARASITIC DISEASES (DIS) & 001-139 & 1 & 0.7 & 0.1 & 4.7 \\
\hline -Other Bacterial Dis & 030-041 & 1 & 0.7 & 0.1 & 4.7 \\
\hline MALIGNANT NEOPLASMS & $140-208,230-234$ & 4 & 2.2 & 0.8 & 6.0 \\
\hline -Lip, Oral Cavity, Pharynx & 140-149 & 1 & 0.7 & 0.1 & 4.7 \\
\hline -Genitourinary & 179-189 & 2 & 1.0 & 0.2 & 4.3 \\
\hline -Lymphatic \& Hematopoietic & $200-208$ & 1 & 0.5 & 0.1 & 3.8 \\
\hline BENIGN \& UNCERTAIN NEOPLASMS & $210-229,235-239$ & 1 & 0.3 & 0.0 & 2.3 \\
\hline ENDOCRINE/METABOLIC/IMMUNITY & 240-279 & 4 & 2.2 & 0.8 & 6.0 \\
\hline -Other Endocrine Gland Dis & $250-259$ & 1 & 0.3 & 0.0 & 2.3 \\
\hline -Other Metabolic \& Immunity Disorders & 270-279 & 3 & 1.9 & 0.6 & 5.8 \\
\hline MENTAL DISORDERS & 290-319 & 5 & 1.8 & 0.7 & 4.5 \\
\hline -Non-Psychotic Disorders & $300-302,306-316$ & 5 & 1.8 & 0.7 & 4.5 \\
\hline NERVOUS SYSTEM (NS) \& SENSE ORGANS & 320-389 & 8 & 4.6 & 2.2 & 9.8 \\
\hline -Disorders of Peripheral NS & $350-359$ & 5 & 2.5 & 1.0 & 6.3 \\
\hline -Disorders of Eye & $360-379$ & 3 & 2.1 & 0.6 & 7.3 \\
\hline CIRCULATORY SYSTEM & $390-459$ & 11 & 4.8 & 2.6 & 8.9 \\
\hline -Hypertensive Dis & $401-405$ & 1 & 0.5 & 0.1 & 3.8 \\
\hline -Ischemic Heart Dis & $410-414$ & 4 & 2.0 & 0.7 & 5.5 \\
\hline -Dis of Pulmonary Circulation & $415-417$ & 1 & 0.3 & 0.0 & 2.3 \\
\hline -Other Heart Dis & $420-429$ & 1 & 0.7 & 0.1 & 4.7 \\
\hline -Cerebrovascular Dis & $430-438$ & 1 & 0.3 & 0.0 & 2.3 \\
\hline -Dis of Arteries \& Capillaries & $440-448$ & 1 & 0.3 & 0.0 & 2.3 \\
\hline -Dis of Veins, Lymphatics, Other & 451-459 & 2 & 0.6 & 0.2 & 2.6 \\
\hline RESPIRATORY SYSTEM & $460-519$ & 16 & 9.3 & 5.4 & 16.1 \\
\hline -Acute Respiratory Infections & $460-466$ & 3 & 1.6 & 0.5 & 5.0 \\
\hline -Other Dis Upper Respiratory Tract & $470-478$ & 5 & 2.6 & 1.0 & 7.3 \\
\hline -Pneumonia \& Influenza & $480-487$ & 7 & 4.7 & 2.1 & 10.7 \\
\hline -Chronic Obstructive Dis & $490-496$ & 1 & 0.3 & 0.0 & 2.3 \\
\hline DIGESTIVE SYSTEM & $520-579$ & 14 & 5.9 & 3.4 & 10.2 \\
\hline -Esophagus, Stomach, Duodenum & $530-537$ & 3 & 1.0 & 0.3 & 3.0 \\
\hline -Hernia & $550-553$ & 3 & 1.5 & 0.5 & 4.9 \\
\hline -Other Intestinal Dis & $560-569$ & 4 & 1.7 & 0.6 & 4.7 \\
\hline -Other Digestive Dis & $570-579$ & 4 & 1.7 & 0.6 & 4.7 \\
\hline GENITOURINARY SYSTEM & \begin{tabular}{|l|}
$580-629$ \\
\end{tabular} & 7 & 3.2 & 1.5 & 7.0 \\
\hline -Other Urinary Dis & $590-599$ & 2 & 1.0 & 0.2 & 4.3 \\
\hline
\end{tabular}

(Continued)

* Only those diagnostic categories with at least one occurrence appear in this table.

**Standardized to age distribution of 2000 U.S. population. 
East Tennessee Technology Park 2008

Absence Data

Appendix K. Age-Adjusted Illness and Injury Rates by Diagnostic Category*

Part 3. Men and Women

\begin{tabular}{|c|c|c|c|c|c|}
\hline & & $\begin{array}{l}\text { Number of } \\
\text { Diagnoses }\end{array}$ & $\begin{array}{c}\text { Age-Adjusted } \\
\text { Rate per } 1,000^{* *}\end{array}$ & $\begin{array}{c}\text { Lower } 95 \% \\
\text { Confidence } \\
\text { Limit per 1,000 }\end{array}$ & $\begin{array}{c}\text { Upper 95\% } \\
\text { Confidence } \\
\text { Limit per 1,000 }\end{array}$ \\
\hline Diagnostic Category & ICD-9-CM Code & \multirow[b]{2}{*}{5} & \multirow[b]{2}{*}{2.3} & \multirow[b]{2}{*}{0.9} & \multirow[b]{2}{*}{5.5} \\
\hline -Other Female Disorders & $617-629$ & & & & \\
\hline $\begin{array}{l}\text { MUSCULOSKELETAL \& CONNECTIVE } \\
\text { TISSUE }\end{array}$ & $710-739$ & 35 & 19.2 & 13.4 & 27.6 \\
\hline -Arthropathies & $710-719$ & 16 & 7.7 & 4.5 & 13.3 \\
\hline -Dorsopathies & $720-724$ & 6 & 3.6 & 1.5 & 8.7 \\
\hline -Rheumatism, Excluding Back & $725-729$ & 9 & 5.7 & 2.8 & 11.6 \\
\hline -Other Dis \& Acquired Deformities & $730-739$ & 4 & 2.1 & 0.8 & 5.7 \\
\hline CONGENITAL ANOMALIES & $740-759$ & 2 & 1.4 & 0.3 & 7.0 \\
\hline $\begin{array}{l}\text { SYMPTOMS, SIGNS, \& ILL-DEFINED } \\
\text { CONDITIONS }\end{array}$ & $780-799$ & 10 & 3.7 & 1.9 & 6.9 \\
\hline -Symptoms & 780-789 & 10 & 3.7 & 1.9 & 6.9 \\
\hline INJURY \& POISONING & $800-999$ & 20 & 9.7 & 6.1 & 15.7 \\
\hline -Fracture - Upper Limb & $810-819$ & 1 & 1.2 & 0.2 & 8.7 \\
\hline -Fracture - Lower Limb & $820-829$ & 1 & 0.5 & 0.1 & 3.8 \\
\hline -Dislocation & 830-839 & 7 & 3.0 & 1.4 & 6.6 \\
\hline -Sprains \& Strains - Back & 846-847 & 1 & 0.3 & 0.0 & 2.3 \\
\hline -Sprains \& Strains - Other & $840-845,848$ & 4 & 1.5 & 0.5 & 4.1 \\
\hline -Open Wound - Head, Neck, Trunk & $870-879$ & 2 & 1.2 & 0.3 & 4.8 \\
\hline -Complications \& Unspecified Injuries & 958-959 & 2 & 0.9 & 0.2 & 3.6 \\
\hline -Unspecified Effects - External Causes & $990-995$ & 2 & 1.1 & 0.3 & 4.3 \\
\hline Total & & 138 & 69.1 & 57.6 & 82.9 \\
\hline
\end{tabular}

* Only those diagnostic categories with at least one occurrence appear in this table.

**Standardized to age distribution of 2000 U.S. population. 
East Tennessee Technology Park 2008

OSHA Data

Appendix L. Number of Workers with at Least One OSHA Event by Gender, Age, and Job Category*

\begin{tabular}{|c|c|c|c|c|c|c|c|c|c|}
\hline \multirow{3}{*}{ Job Category } & \multicolumn{3}{|c|}{ Women } & \multicolumn{5}{|c|}{ Men } & \multirow[b]{3}{*}{ TOTAI } \\
\hline & \multicolumn{2}{|c|}{ Age Group } & \multirow[b]{2}{*}{ TOTAL } & \multicolumn{4}{|c|}{ Age Group } & \multirow[b]{2}{*}{ TOTAL } & \\
\hline & $40-49$ & $50+$ & & $16-29$ & $30-39$ & $40-49$ & $50+$ & & \\
\hline Service & 1 & 3 & 4 & 2 & 0 & 4 & 6 & 12 & 16 \\
\hline Crafts & 0 & 0 & 0 & 0 & 4 & 3 & 3 & 10 & 10 \\
\hline Line Operators & 0 & 1 & 1 & 0 & 0 & 1 & 4 & 5 & 6 \\
\hline TOTAL & 1 & 4 & 5 & 2 & 4 & 8 & 13 & 27 & 32 \\
\hline
\end{tabular}

*Only those job categories and gender/age combinations with at least one OSHA event appear in this table.

Appendix M. Total Number of Workdays Lost or with Restricted Activity from OSHA Events by Gender and Age

\begin{tabular}{|c|c|c|c|c|c|c|c|c|c|}
\hline \multirow[b]{2}{*}{ Age Group } & \multicolumn{3}{|c|}{ Women } & \multicolumn{3}{|c|}{ Men } & \multicolumn{3}{|c|}{ TOTAL } \\
\hline & $\begin{array}{c}\text { Number of } \\
\text { Events }\end{array}$ & $\begin{array}{c}\text { Days } \\
\text { Restricted }\end{array}$ & Days Lost & $\begin{array}{l}\text { Number of } \\
\text { Events }\end{array}$ & $\begin{array}{c}\text { Days } \\
\text { Restricted }\end{array}$ & Days Lost & $\begin{array}{c}\text { Number of } \\
\text { Events }\end{array}$ & $\begin{array}{c}\text { Days } \\
\text { Restricted }\end{array}$ & Days Lost \\
\hline $16-29$ & 0 & 0 & 0 & 2 & 0 & 0 & 2 & 0 & 0 \\
\hline $30-39$ & 0 & 0 & 0 & 4 & 34 & 15 & 4 & 34 & 15 \\
\hline $40-49$ & 1 & 47 & 0 & 8 & 111 & 0 & 9 & 158 & 0 \\
\hline $50+$ & 4 & 18 & 0 & 13 & 64 & 316 & 17 & 82 & 316 \\
\hline TOTAL & 5 & 65 & 0 & 27 & 209 & 331 & 32 & 274 & 331 \\
\hline
\end{tabular}

Appendix N. Total Number of Workdays Lost or with Restricted Activity from OSHA Events by Gender and Job Category*

\begin{tabular}{|c|c|c|c|c|c|c|c|c|c|}
\hline \multirow[b]{2}{*}{ Job Category } & \multicolumn{3}{|c|}{ Women } & \multicolumn{3}{|c|}{ Men } & \multicolumn{3}{|c|}{ TOTAL } \\
\hline & $\begin{array}{l}\text { Number of } \\
\text { Events }\end{array}$ & $\begin{array}{c}\text { Days } \\
\text { Restricted }\end{array}$ & Days Lost & $\begin{array}{c}\text { Number of } \\
\text { Events }\end{array}$ & $\begin{array}{c}\text { Days } \\
\text { Restricted }\end{array}$ & Days Lost & $\begin{array}{l}\text { Number of } \\
\text { Events }\end{array}$ & $\begin{array}{c}\text { Days } \\
\text { Restricted }\end{array}$ & Days Lost \\
\hline Crafts & 0 & 0 & 0 & 10 & 90 & 15 & 10 & 90 & 15 \\
\hline Line Operators & 1 & 15 & 0 & 5 & 6 & 273 & 6 & 21 & 273 \\
\hline
\end{tabular}

*Only those job categories with at least one OSHA event appear in this table. 
East Tennessee Technology Park 2008

OSHA Data

Appendix O. Number of Diagnoses in Each Diagnostic Category by Gender and Age*

\begin{tabular}{|c|c|c|c|c|}
\hline & & \multicolumn{3}{|c|}{ Women } \\
\hline & & \multicolumn{2}{|c|}{ Age Group } & \multirow[b]{2}{*}{ TOTAL } \\
\hline & & $40-49$ & $50+$ & \\
\hline Diagnostic Category & ICD-9-CM code & \multirow[b]{2}{*}{0} & \multirow[b]{2}{*}{0} & \multirow[b]{2}{*}{0} \\
\hline NERVOUS SYSTEM (NS) \& SENSE ORGANS & 320-389 & & & \\
\hline -Dis of Ear \& Mastoid & 380-389 & 0 & 0 & 0 \\
\hline INJURY \& POISONING & 800-999 & 1 & 4 & 5 \\
\hline -Fracture - Lower Limb & $820-829$ & 0 & 0 & 0 \\
\hline -Sprains \& Strains - Back & 846-847 & 0 & 0 & 0 \\
\hline -Sprains \& Strains - Other & $840-845,848$ & 1 & 2 & 3 \\
\hline -Open Wound - Head, Neck, Trunk & 870-879 & 0 & 0 & 0 \\
\hline -Open Wound - Upper Limb & $880-887$ & 0 & 1 & 1 \\
\hline -Open Wound - Lower Limb & 890-897 & 0 & 0 & 0 \\
\hline -Superficial Injury & 910-919 & 0 & 0 & 0 \\
\hline -Contusion & $920-924$ & 0 & 1 & 1 \\
\hline -Burns & $940-949$ & 0 & 0 & 0 \\
\hline
\end{tabular}

\begin{tabular}{|c|c|c|c|c|c|c|c|}
\hline & & \multicolumn{5}{|c|}{ Men } & \multirow[b]{3}{*}{ TOTAI } \\
\hline & & \multicolumn{4}{|c|}{ Age Group } & \multirow[b]{2}{*}{ TOTAL } & \\
\hline & & $16-29$ & 30 - 39 & $40-49$ & $\mathbf{5 0 +}$ & & \\
\hline Diagnostic Category & ICD-9-CM code & & & & & & \\
\hline NERVOUS SYSTEM (NS) \& SENSE ORGANS & $320-389$ & 0 & 0 & 0 & 1 & 1 & 1 \\
\hline -Dis of Ear \& Mastoid & 380-389 & 0 & 0 & 0 & 1 & 1 & 1 \\
\hline INJURY \& POISONING & $800-999$ & 2 & 4 & 8 & 12 & 26 & 31 \\
\hline -Fracture - Lower Limb & $820-829$ & 0 & 0 & 0 & 1 & 1 & 1 \\
\hline -Sprains \& Strains - Back & 846-847 & 0 & 0 & 0 & 2 & 2 & 2 \\
\hline -Sprains \& Strains - Other & $840-845,848$ & 0 & 1 & 4 & 4 & 9 & 12 \\
\hline -Open Wound - Head, Neck, Trunk & $870-879$ & 1 & 0 & 1 & 1 & 3 & 3 \\
\hline -Open Wound - Upper Limb & 880-887 & 0 & 1 & 1 & 2 & 4 & 5 \\
\hline -Open Wound - Lower Limb & $890-897$ & 0 & 0 & 1 & 1 & 2 & 2 \\
\hline -Superficial Injury & 910-919 & 0 & 1 & 1 & 1 & 3 & 3 \\
\hline -Contusion & $920-924$ & 0 & 1 & 0 & 0 & 1 & 2 \\
\hline -Burns & 940-949 & 1 & 0 & 0 & 0 & 1 & 1 \\
\hline
\end{tabular}

\begin{tabular}{|c|c|c|c|c|c|c|c|c|c|}
\hline & \multicolumn{3}{|c|}{ Women } & \multicolumn{5}{|c|}{ Men } & \multirow[b]{3}{*}{ TOTAL } \\
\hline & \multicolumn{2}{|c|}{ Age Group } & \multirow[b]{2}{*}{ TOTAL } & \multicolumn{4}{|c|}{ Age Group } & \multirow[b]{2}{*}{ TOTAL } & \\
\hline & $40-49$ & $50+$ & & $16-29$ & 30 - 39 & $40-49$ & $50+$ & & \\
\hline Diagnostic Category & \multirow[b]{2}{*}{1} & \multirow[b]{2}{*}{4} & \multirow[b]{2}{*}{5} & \multirow[b]{2}{*}{2} & \multirow[b]{2}{*}{4} & \multirow[b]{2}{*}{8} & \multirow[b]{2}{*}{13} & \multirow[b]{2}{*}{27} & \multirow[b]{2}{*}{32} \\
\hline Total & & & & & & & & & \\
\hline
\end{tabular}

*Only those diagnostic categories and gender/age combinations with at least one OSHA event appear in this table. 
East Tennessee Technology Park 2008

OSHA Data

Appendix P. Number of Workdays Lost or with Restricted Activity in Each Diagnostic Category by Gender and Age*

\begin{tabular}{|c|c|c|c|c|c|}
\hline & & \multicolumn{4}{|c|}{ Women } \\
\hline & & \multicolumn{4}{|c|}{ Age Group } \\
\hline & & \multicolumn{2}{|c|}{$40-49$} & \multicolumn{2}{|c|}{$50+$} \\
\hline & & $\begin{array}{c}\text { Days } \\
\text { Restricted }\end{array}$ & Days Lost & $\begin{array}{c}\text { Days } \\
\text { Restricted }\end{array}$ & Days Lost \\
\hline Diagnostic Category & ICD-9-CM Codes & \multirow[b]{2}{*}{47} & \multirow[b]{2}{*}{0} & \multirow[b]{2}{*}{15} & \multirow[b]{2}{*}{0} \\
\hline -Sprains \& Strains - Other & $840-845,848$ & & & & \\
\hline -Open Wound - Upper Limb & 880-887 & 0 & 0 & 3 & 0 \\
\hline -Contusion & $920-924$ & 0 & 0 & 0 & 0 \\
\hline
\end{tabular}

\begin{tabular}{|c|c|c|c|c|c|c|c|c|c|}
\hline & & \multicolumn{8}{|c|}{ Men } \\
\hline & & \multicolumn{8}{|c|}{ Age Group } \\
\hline & & \multicolumn{2}{|c|}{$16-29$} & \multicolumn{2}{|c|}{$30-39$} & \multicolumn{2}{|c|}{$40-49$} & \multicolumn{2}{|c|}{$\mathbf{5 0 +}$} \\
\hline & & \begin{tabular}{c|} 
Days \\
Restricted
\end{tabular} & $\begin{array}{l}\text { Days } \\
\text { Lost }\end{array}$ & $\begin{array}{c}\text { Days } \\
\text { Restricted }\end{array}$ & $\begin{array}{l}\text { Days } \\
\text { Lost }\end{array}$ & $\begin{array}{c}\text { Days } \\
\text { Restricted }\end{array}$ & $\begin{array}{l}\text { Days } \\
\text { Lost }\end{array}$ & $\begin{array}{c}\text { Days } \\
\text { Restricted }\end{array}$ & $\begin{array}{l}\text { Days } \\
\text { Lost }\end{array}$ \\
\hline Diagnostic Category & ICD-9-CM Codes & & & & & & & & \\
\hline -Dis of Ear \& Mastoid & $380-389$ & 0 & 0 & 0 & 0 & 0 & 0 & 0 & 0 \\
\hline -Fracture - Lower Limb & $820-829$ & 0 & 0 & 0 & 0 & 0 & 0 & 0 & 0 \\
\hline -Sprains \& Strains - Back & 846-847 & 0 & 0 & 0 & 0 & 0 & 0 & 16 & 141 \\
\hline -Sprains \& Strains - Other & $840-845,848$ & 0 & 0 & 34 & 15 & 108 & 0 & 37 & 132 \\
\hline -Open Wound - Head, Neck, Trunk & $870-879$ & 0 & 0 & 0 & 0 & 0 & 0 & 0 & 43 \\
\hline -Open Wound - Upper Limb & 880-887 & 0 & 0 & 0 & 0 & 0 & 0 & 11 & 0 \\
\hline -Open Wound - Lower Limb & $890-897$ & 0 & 0 & 0 & 0 & 0 & 0 & 0 & 0 \\
\hline -Superficial Injury & $910-919$ & 0 & 0 & 0 & 0 & 3 & 0 & 0 & 0 \\
\hline -Contusion & \begin{tabular}{|c|}
$920-924$ \\
\end{tabular} & 0 & 0 & 0 & 0 & 0 & 0 & 0 & 0 \\
\hline -Burns & \begin{tabular}{|c|}
$940-949$ \\
\end{tabular} & 0 & 0 & 0 & 0 & 0 & 0 & 0 & 0 \\
\hline
\end{tabular}

*OSHA events with >1 ICD-9-CM code in the same diagnostic category were counted only once. Only those diagnostic categories and gender/age combinations with at least one occurrence appear in this table. 
East Tennessee Technology Park 2008

OSHA Data

Appendix Q. Number of Occurrences in Each Accident Category by Gender and Age*

\begin{tabular}{|c|c|c|c|c|c|c|c|}
\hline & & \multicolumn{5}{|c|}{ Men } & \multirow[b]{3}{*}{ TOTAL } \\
\hline & & \multicolumn{4}{|c|}{ Age Group } & \multirow[b]{2}{*}{ TOTAL } & \\
\hline & & $16-29$ & 30 - 39 & $40-49$ & $50+$ & & \\
\hline Type of Accident & E Codes & & & & & & \\
\hline Natural/Environmental Factors & E900-E909 & 0 & 1 & 1 & 0 & 2 & 2 \\
\hline Other Accidents & E916-E928 & 2 & 0 & 1 & 2 & 5 & 5 \\
\hline
\end{tabular}

*Only those accident types and gender/age combinations with at least one occurrence appear in this table. 
East Tennessee Technology Park 2008

OSHA Data

Appendix R. Number of Workdays Lost or with Restricted Activity in Each Accident Category by Gender and Age*

\begin{tabular}{|c|c|c|c|c|c|c|c|c|c|}
\hline & & \multicolumn{8}{|c|}{ Men } \\
\hline & & \multicolumn{8}{|c|}{ Age Group } \\
\hline & & \multicolumn{2}{|c|}{$16-29$} & \multicolumn{2}{|c|}{$30-39$} & \multicolumn{2}{|c|}{$40-49$} & \multicolumn{2}{|c|}{$50+$} \\
\hline & & $\begin{array}{c}\text { Days } \\
\text { Restricted }\end{array}$ & $\begin{array}{l}\text { Days } \\
\text { Lost }\end{array}$ & $\begin{array}{c}\text { Days } \\
\text { Restricted }\end{array}$ & $\begin{array}{l}\text { Days } \\
\text { Lost }\end{array}$ & $\begin{array}{c}\text { Days } \\
\text { Restricted }\end{array}$ & $\begin{array}{l}\text { Days } \\
\text { Lost }\end{array}$ & $\begin{array}{c}\text { Days } \\
\text { Restricted }\end{array}$ & $\begin{array}{l}\text { Days } \\
\text { Lost }\end{array}$ \\
\hline Type of Accident & E Codes & \multirow[b]{2}{*}{0} & \multirow[b]{2}{*}{0} & \multirow[b]{2}{*}{0} & \multirow[b]{2}{*}{0} & \multirow[b]{2}{*}{3} & \multirow[b]{2}{*}{0} & \multirow[b]{2}{*}{0} & \multirow[b]{2}{*}{0} \\
\hline Natural/Environmental Factors & E900-E909 & & & & & & & & \\
\hline Other Accidents & E916-E928 & 0 & 0 & 0 & 0 & 0 & 0 & 0 & 43 \\
\hline
\end{tabular}

*OSHA events with $>1 \mathrm{E}$ code in the same accident type were counted only once. Only those accident types and gender/age combinations with at least one occurrence appear in this table. 
East Tennessee Technology Park 2008

OSHA Data

Appendix S. Number of Diagnoses in Each Diagnostic Category by Gender and Job Category*

\begin{tabular}{|c|c|c|c|c|}
\hline & & \multicolumn{3}{|c|}{ Women } \\
\hline & & \multicolumn{2}{|c|}{ Job Category } & \multirow[b]{2}{*}{ TOTAL } \\
\hline & & Service & Line Operators & \\
\hline Diagnostic Category & ICD-9-CM Code & \multirow[b]{2}{*}{4} & \multirow[b]{2}{*}{1} & \multirow[b]{2}{*}{5} \\
\hline INJURY \& POISONING & 800-999 & & & \\
\hline $\begin{array}{l}\text {-Sprains \& Strains - } \\
\text { Other }\end{array}$ & $840-845,848$ & 2 & 1 & 3 \\
\hline $\begin{array}{l}\text {-Open Wound - Upper } \\
\text { Limb }\end{array}$ & $880-887$ & 1 & 0 & 1 \\
\hline -Contusion & 920-924 & 1 & 0 & 1 \\
\hline
\end{tabular}

\begin{tabular}{|c|c|c|c|}
\hline & \multicolumn{3}{|c|}{ Women } \\
\hline & \multicolumn{2}{|c|}{ Job Category } & \multirow[b]{2}{*}{ TOTAL } \\
\hline & Service & Line Operators & \\
\hline Diagnostic Category & \multirow[b]{2}{*}{4} & \multirow[b]{2}{*}{1} & \multirow[b]{2}{*}{5} \\
\hline Total & & & \\
\hline
\end{tabular}

\begin{tabular}{|c|c|c|c|c|c|}
\hline & & \multicolumn{4}{|c|}{ Men } \\
\hline & & \multicolumn{3}{|c|}{ Job Category } & \multirow[b]{2}{*}{ TOTAL } \\
\hline & & Service & Crafts & Line Operators & \\
\hline Diagnostic Category & ICD-9-CM Code & & & & \\
\hline $\begin{array}{l}\text { NERVOUS SYSTEM } \\
\text { (NS) \& SENSE ORGANS }\end{array}$ & $320-389$ & 0 & 1 & 0 & 1 \\
\hline -Dis of Ear \& Mastoid & $380-389$ & 0 & 1 & 0 & 1 \\
\hline INJURY \& POISONING & $800-999$ & 12 & 9 & 5 & 26 \\
\hline -Fracture - Lower Limb & $820-829$ & 0 & 0 & 1 & 1 \\
\hline -Sprains \& Strains - Back & 846-847 & 0 & 1 & 1 & 2 \\
\hline $\begin{array}{l}\text {-Sprains \& Strains - } \\
\text { Other }\end{array}$ & $840-845,848$ & 4 & 2 & 3 & 9 \\
\hline $\begin{array}{l}\text {-Open Wound - Head, } \\
\text { Neck, Trunk }\end{array}$ & 870-879 & 2 & 1 & 0 & 3 \\
\hline $\begin{array}{l}\text {-Open Wound - Upper } \\
\text { Limb }\end{array}$ & $880-887$ & 2 & 2 & 0 & 4 \\
\hline $\begin{array}{l}\text {-Open Wound - Lower } \\
\text { Limb }\end{array}$ & 890-897 & 2 & 0 & 0 & 2 \\
\hline -Superficial Injury & 910-919 & 1 & 2 & 0 & 3 \\
\hline -Contusion & $920-924$ & 0 & 1 & 0 & 1 \\
\hline -Burns & 940-949 & 1 & 0 & 0 & 1 \\
\hline
\end{tabular}

*Only those diagnostic categories and gender/job category combinations with at least one occurrence appear in this table. 
East Tennessee Technology Park 2008

OSHA Data

Appendix S. Number of Diagnoses in Each Diagnostic Category by Gender and Job Category*

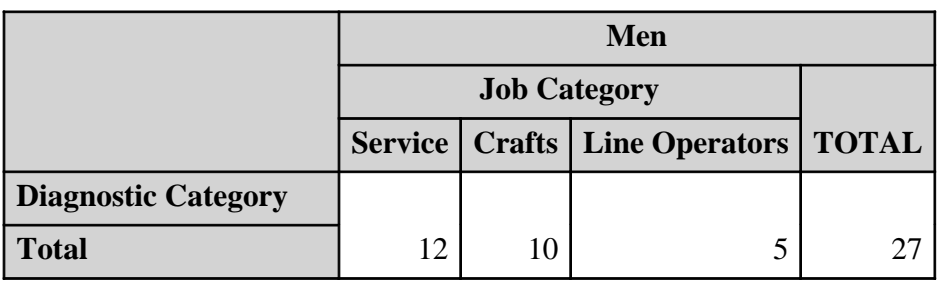

*Only those diagnostic categories and gender/job category combinations with at least one occurrence appear in this table. 
East Tennessee Technology Park 2008

OSHA Data

Appendix T. Number of Workdays Lost or with Restricted Activity in Each Diagnostic Category by Gender and Job Category*

\begin{tabular}{|c|c|c|c|c|c|}
\hline & & \multicolumn{4}{|c|}{ Women } \\
\hline & & \multicolumn{4}{|c|}{ Job Category } \\
\hline & & \multicolumn{2}{|c|}{ Service } & \multicolumn{2}{|c|}{ Line Operators } \\
\hline & & $\begin{array}{c}\text { Days } \\
\text { Restricted }\end{array}$ & Days Lost & $\begin{array}{c}\text { Days } \\
\text { Restricted }\end{array}$ & Days Lost \\
\hline Diagnostic Category & ICD-9-CM Codes & \multirow[b]{2}{*}{47} & \multirow[b]{2}{*}{0} & \multirow[b]{2}{*}{15} & \multirow[b]{2}{*}{0} \\
\hline -Sprains \& Strains - Other & $840-845,848$ & & & & \\
\hline -Open Wound - Upper Limb & $880-887$ & 3 & 0 & 0 & 0 \\
\hline -Contusion & $920-924$ & 0 & 0 & 0 & 0 \\
\hline
\end{tabular}

\begin{tabular}{|c|c|c|c|c|c|c|c|}
\hline & & \multicolumn{6}{|c|}{ Men } \\
\hline & & \multicolumn{6}{|c|}{ Job Category } \\
\hline & & \multicolumn{2}{|c|}{ Service } & \multicolumn{2}{|c|}{ Crafts } & \multicolumn{2}{|c|}{ Line Operators } \\
\hline & & $\begin{array}{c}\text { Days } \\
\text { Restricted }\end{array}$ & Days Lost & \begin{tabular}{|c|} 
Days \\
Restricted
\end{tabular} & Days Lost & \begin{tabular}{|c|} 
Days \\
Restricted
\end{tabular} & Days Lost \\
\hline Diagnostic Category & ICD-9-CM Codes & \multirow[b]{2}{*}{0} & \multirow[b]{2}{*}{0} & \multirow[b]{2}{*}{0} & \multirow[b]{2}{*}{0} & \multirow[b]{2}{*}{0} & \multirow[b]{2}{*}{0} \\
\hline -Dis of Ear \& Mastoid & $380-389$ & & & & & & \\
\hline -Fracture - Lower Limb & $820-829$ & 0 & 0 & 0 & 0 & 0 & 0 \\
\hline -Sprains \& Strains - Back & 846-847 & 0 & 0 & 16 & 0 & 0 & 141 \\
\hline -Sprains \& Strains - Other & $840-845,848$ & 102 & 0 & 71 & 15 & 6 & 132 \\
\hline -Open Wound - Head, Neck, Trunk & $870-879$ & 0 & 43 & 0 & 0 & 0 & 0 \\
\hline -Open Wound - Upper Limb & 880-887 & 11 & 0 & 0 & 0 & 0 & 0 \\
\hline -Open Wound - Lower Limb & 890-897 & 0 & 0 & 0 & 0 & 0 & 0 \\
\hline -Superficial Injury & 910-919 & 0 & 0 & 3 & 0 & 0 & 0 \\
\hline -Contusion & $920-924$ & 0 & 0 & 0 & 0 & 0 & 0 \\
\hline -Burns & 940-949 & 0 & 0 & 0 & 0 & 0 & 0 \\
\hline
\end{tabular}

*OSHA events with >1 ICD-9-CM code in the same diagnostic category were counted only once. Only those diagnostic categories and gender/job category combinations with at least one occurrence appear in this table. 
East Tennessee Technology Park 2008

OSHA Data

Appendix U. Number of Occurrences in Each Accident Category by Gender and Job Category*

\begin{tabular}{|c|c|c|c|c|}
\hline & & \multicolumn{3}{|c|}{ Men } \\
\hline & & \multicolumn{2}{|c|}{ Job Category } & \multirow[b]{2}{*}{ TOTAL } \\
\hline & & Service & Crafts & \\
\hline Type of Accident & E CODES & \multirow[b]{2}{*}{0} & \multirow[b]{2}{*}{2} & \multirow[b]{2}{*}{2} \\
\hline Natural/Environmental Factors & E900-E909 & & & \\
\hline Other Accidents & E916-E928 & 4 & 1 & 5 \\
\hline
\end{tabular}

*Only those accident types and gender/job category combinations with at least one occurrence appear in this table. 
East Tennessee Technology Park 2008

OSHA Data

Appendix V. Number of Workdays Lost or with Restricted Activity in Each Accident Category by Gender and Job Category*

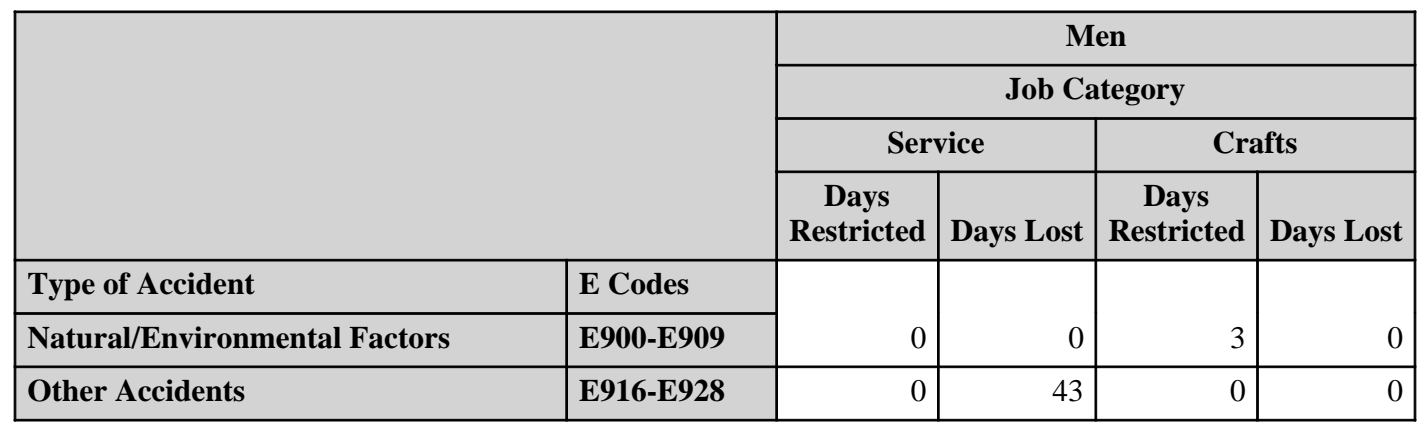

*OSHA events with >1 E code in the same accident type were counted only once. Only those accident types and gender/job category combinations with at least one occurrence appear in this table. 
East Tennessee Technology Park 2008

OSHA Data

Appendix W. Age-Adjusted OSHA Illness and Injury Rates by Diagnostic Category*

Part 1. Men

\begin{tabular}{|c|c|c|c|c|c|}
\hline & & $\begin{array}{c}\text { Number of } \\
\text { Diagnoses }\end{array}$ & $\begin{array}{c}\text { Age-Adjusted } \\
\text { Rate per 1,000*** }\end{array}$ & $\begin{array}{c}\text { Lower } 95 \% \\
\text { Confidence } \\
\text { Limit per 1,000 }\end{array}$ & $\begin{array}{c}\text { Upper 95\% } \\
\text { Confidence } \\
\text { Limit per 1,000 }\end{array}$ \\
\hline Diagnostic Category & ICD-9-CM Code & \multirow[b]{2}{*}{1} & \multirow[b]{2}{*}{0.8} & \multirow[b]{2}{*}{0.1} & \multirow[b]{2}{*}{5.4} \\
\hline NERVOUS SYSTEM (NS) \& SENSE ORGANS & 320-389 & & & & \\
\hline -Dis of Ear \& Mastoid & 380-389 & 1 & 0.8 & 0.1 & 5.4 \\
\hline INJURY \& POISONING & $800-999$ & 26 & 21.4 & 14.0 & 32.5 \\
\hline -Fracture - Lower Limb & $820-829$ & 1 & 0.8 & 0.1 & 5.4 \\
\hline -Sprains \& Strains - Back & 846-847 & 2 & 1.2 & 0.3 & 5.0 \\
\hline -Sprains \& Strains - Other & $840-845,848$ & 9 & 6.9 & 3.5 & 13.9 \\
\hline -Open Wound - Head, Neck, Trunk & 870-879 & 3 & 2.7 & 0.8 & 9.3 \\
\hline -Open Wound - Upper Limb & 880-887 & 4 & 3.1 & 1.0 & 9.3 \\
\hline -Open Wound - Lower Limb & 890-897 & 2 & 1.2 & 0.3 & 5.1 \\
\hline -Superficial Injury & 910-919 & 3 & 2.6 & 0.8 & 9.2 \\
\hline -Contusion & $920-924$ & 1 & 1.4 & 0.2 & 10.2 \\
\hline -Burns & $940-949$ & 1 & 1.4 & 0.2 & 10.2 \\
\hline Total & & 27 & 22.1 & 14.7 & 33.4 \\
\hline
\end{tabular}

*Only those diagnostic categories with at least one occurrence appear in this table.

**Standardized to age distribution of 2000 U.S. population. 
East Tennessee Technology Park 2008

OSHA Data

Appendix W. Age-Adjusted OSHA Illness and Injury Rates by Diagnostic Category*

Part 2. Women

\begin{tabular}{|c|c|c|c|c|c|}
\hline & & $\begin{array}{c}\text { Number of } \\
\text { Diagnoses }\end{array}$ & $\begin{array}{c}\text { Age-Adjusted } \\
\text { Rate per } 1,000^{* *}\end{array}$ & $\begin{array}{c}\text { Lower } 95 \% \\
\text { Confidence } \\
\text { Limit per } 1,000\end{array}$ & $\begin{array}{c}\text { Upper } 95 \% \\
\text { Confidence } \\
\text { Limit per 1,000 }\end{array}$ \\
\hline Diagnostic Category & ICD-9-CM Code & \multirow[b]{2}{*}{5} & \multirow[b]{2}{*}{9.2} & \multirow[b]{2}{*}{3.4} & \multirow[b]{2}{*}{25.2} \\
\hline INJURY \& POISONING & 800-999 & & & & \\
\hline -Sprains \& Strains - Other & $840-845,848$ & 3 & 4.1 & 1.3 & 13.1 \\
\hline -Open Wound - Upper Limb & 880-887 & 1 & 1.2 & 0.2 & 8.6 \\
\hline -Contusion & $920-924$ & 1 & 3.9 & 0.5 & 27.6 \\
\hline Total & & 5 & 9.2 & 3.4 & 25.2 \\
\hline
\end{tabular}

*Only those diagnostic categories with at least one occurrence appear in this table.

**Standardized to age distribution of 2000 U.S. population. 
East Tennessee Technology Park 2008

OSHA Data

Appendix W. Age-Adjusted OSHA Illness and Injury Rates by Diagnostic Category*

Part 3. Men and Women

\begin{tabular}{|c|c|c|c|c|c|}
\hline & & $\begin{array}{l}\text { Number of } \\
\text { Diagnoses }\end{array}$ & $\begin{array}{c}\text { Age-Adjusted } \\
\text { Rate per 1,000*** }\end{array}$ & $\begin{array}{c}\text { Lower } 95 \% \\
\text { Confidence } \\
\text { Limit per 1,000 }\end{array}$ & $\begin{array}{c}\text { Upper } 95 \% \\
\text { Confidence } \\
\text { Limit per 1,000 }\end{array}$ \\
\hline Diagnostic Category & ICD-9-CM Code & \multirow[b]{2}{*}{1} & \multirow[b]{2}{*}{0.7} & \multirow[b]{2}{*}{0.1} & \multirow[b]{2}{*}{4.7} \\
\hline NERVOUS SYSTEM (NS) \& SENSE ORGANS & $320-389$ & & & & \\
\hline -Dis of Ear \& Mastoid & $380-389$ & 1 & 0.7 & 0.1 & 4.7 \\
\hline INJURY \& POISONING & $800-999$ & 31 & 18.6 & 12.6 & 27.6 \\
\hline -Fracture - Lower Limb & $820-829$ & 1 & 0.7 & 0.1 & 4.7 \\
\hline -Sprains \& Strains - Back & 846-847 & 2 & 1.0 & 0.2 & 4.3 \\
\hline -Sprains \& Strains - Other & $840-845,848$ & 12 & 6.4 & 3.5 & 11.8 \\
\hline -Open Wound - Head, Neck, Trunk & 870-879 & 3 & 2.1 & 0.6 & 7.6 \\
\hline -Open Wound - Upper Limb & 880-887 & 5 & 2.6 & 1.0 & 7.3 \\
\hline -Open Wound - Lower Limb & 890-897 & 2 & 0.9 & 0.2 & 3.6 \\
\hline -Superficial Injury & $910-919$ & 3 & 2.0 & 0.6 & 7.1 \\
\hline -Contusion & $920-924$ & 2 & 1.8 & 0.4 & 7.4 \\
\hline -Burns & $940-949$ & 1 & 1.2 & 0.2 & 8.7 \\
\hline Total & & 32 & 19.3 & 13.1 & 28.3 \\
\hline
\end{tabular}

*Only those diagnostic categories with at least one occurrence appear in this table.

**Standardized to age distribution of 2000 U.S. population. 